\title{
Hybrid and Relaxed Mann Iterations for General Systems of Variational Inequalities and Nonexpansive Mappings
}

\author{
L. C. Ceng, ${ }^{1}$ A. E. Al-Mazrooei, ${ }^{2}$ A. A. N. Abdou, ${ }^{2}$ and A. Latif ${ }^{2}$ \\ ${ }^{1}$ Department of Mathematics, Shanghai Normal University and Scientific Computing Key Laboratory of Shanghai Universities, \\ Shanghai 200234, China \\ ${ }^{2}$ Department of Mathematics, King Abdulaziz University, P.O. Box 80203, Jeddah 21589, Saudi Arabia
}

Correspondence should be addressed to A. Latif; latifmath@yahoo.com

Received 4 August 2013; Accepted 10 August 2013

Academic Editor: Jen-Chih Yao

Copyright ( 92013 L. C. Ceng et al. This is an open access article distributed under the Creative Commons Attribution License, which permits unrestricted use, distribution, and reproduction in any medium, provided the original work is properly cited.

\begin{abstract}
We introduce hybrid and relaxed Mann iteration methods for a general system of variational inequalities with solutions being also common solutions of a countable family of variational inequalities and common fixed points of a countable family of nonexpansive mappings in real smooth and uniformly convex Banach spaces. Here, the hybrid and relaxed Mann iteration methods are based on Korpelevich's extragradient method, viscosity approximation method, and Mann iteration method. Under suitable assumptions, we derive some strong convergence theorems for hybrid and relaxed Mann iteration algorithms not only in the setting of uniformly convex and 2-uniformly smooth Banach space but also in a uniformly convex Banach space having a uniformly Gateaux differentiable norm. The results presented in this paper improve, extend, supplement, and develop the corresponding results announced in the earlier and very recent literature.
\end{abstract}

\section{Introduction}

Let $X$ be a real Banach space whose dual space is denoted by $X^{*}$. The normalized duality mapping $J: X \rightarrow 2^{X^{*}}$ is defined by

$$
J(x)=\left\{x^{*} \in X^{*}:\left\langle x, x^{*}\right\rangle=\|x\|^{2}=\left\|x^{*}\right\|^{2}\right\}, \quad \forall x \in X,
$$

where $\langle\cdot, \cdot\rangle$ denotes the generalized duality pairing. It is an immediate consequence of the Hahn-Banach theorem that $J(x)$ is nonempty for each $x \in X$. Let $C$ be a nonempty closed convex subset of $X$. A mapping $T: C \rightarrow C$ is called nonexpansive if $\|T x-T y\| \leq\|x-y\|$ for every $x, y \in C$. The set of fixed points of $T$ is denoted by $\operatorname{Fix}(T)$. We use the notation $\rightarrow$ to indicate the weak convergence and the one $\rightarrow$ to indicate the strong convergence. A mapping $A: C \rightarrow X$ is said to be

(i) accretive if for each $x, y \in C$ there exists $j(x-y) \in$ $J(x-y)$ such that

$$
\langle A x-A y, j(x-y)\rangle \geq 0
$$

(ii) $\alpha$-strongly accretive if for each $x, y \in C$ there exists $j(x-y) \in J(x-y)$ such that

$$
\langle A x-A y, j(x-y)\rangle \geq \alpha\|x-y\|^{2},
$$

for some $\alpha \in(0,1)$;

(iii) $\beta$-inverse strongly accretive if, for each $x, y \in C$, there exists $j(x-y) \in J(x-y)$ such that

$$
\langle A x-A y, j(x-y)\rangle \geq \beta\|A x-A y\|^{2},
$$

for some $\beta>0$

(iv) $\lambda$-strictly pseudocontractive [1] (see also [2]) if for each $x, y \in C$ there exists $j(x-y) \in J(x-y)$ such that

$$
\langle A x-A y, j(x-y)\rangle \leq\|x-y\|^{2}-\lambda\|x-y-(A x-A y)\|^{2}
$$

for some $\lambda \in(0,1)$. 
It is worth emphasizing that the definition of the inverse strongly accretive mapping is based on that of the inverse strongly monotone mapping, which was studied by so many authors; see, for example, [3-5]. Let $U=\{x \in X:\|x\|=1\}$ denote the unite sphere of $X$. A Banach space $X$ is said to be uniformly convex if, for each $\epsilon \in(0,2]$, there exists $\delta>0$ such that, for all $x, y \in U$,

$$
\|x-y\| \geq \epsilon \Longrightarrow \frac{\|x+y\|}{2} \leq 1-\delta .
$$

It is known that a uniformly convex Banach space is reflexive and strict convex. A Banach space $X$ is said to be smooth if the limit

$$
\lim _{t \rightarrow 0} \frac{\|x+t y\|-\|x\|}{t}
$$

exists for all $x, y \in U$; in this case, $X$ is also said to have a Gateaux differentiable norm. $X$ is said to have a uniformly, Gateaux differentiable norm if, for each $y \in U$, the limit is attained uniformly for $x \in U$. Moreover, it is said to be uniformly smooth if this limit is attained uniformly for $x, y \in$ $U$. The norm of $X$ is said to be the Frechet differential if for each $x \in U$, this limit is attained uniformly for $y \in U$. In the meantime, we define a function $\rho:[0, \infty) \rightarrow[0, \infty)$ called the modulus of smoothness of $X$ as follows:

$$
\begin{gathered}
\rho(\tau)=\sup \left\{\frac{1}{2}(\|x+y\|+\|x-y\|)-1: x, y \in X,\right. \\
\|x\|=1,\|y\|=\tau\} .
\end{gathered}
$$

It is known that $X$ is uniformly smooth if and only if $\lim _{\tau \rightarrow 0} \rho(\tau) / \tau=0$. Let $q$ be a fixed real number with $1<q \leq$ 2. Then, a Banach space $X$ is said to be $q$-uniformly smooth if there exists a constant $c>0$ such that $\rho(\tau) \leq c \tau^{q}$ for all $\tau>0$. As pointed out in [6], no Banach space is $q$-uniformly smooth for $q>2$. In addition, it is also known that $J$ is single valued if and only if $X$ is smooth, whereas if $X$ is uniformly smooth, then the mapping $J$ is norm-to-norm uniformly continuous on bounded subsets of $X$. If $X$ has a uniformly Gateaux differentiable norm, then the duality mapping $J$ is norm-toweak ${ }^{*}$ uniformly continuous on bounded subsets of $X$.

Recently, Yao et al. [7] combined the viscosity approximation method and Mann iteration method and gave the following hybrid viscosity approximation method.

Let $C$ be a nonempty closed convex subset of a real uniformly smooth Banach space $X, T: C \rightarrow C$ a nonexpansive mapping with $\operatorname{Fix}(T) \neq \emptyset$, and $f: C \rightarrow C$ a contraction with coefficient $\rho \in(0,1)$. For an arbitrary $x_{0} \in C$, define $\left\{x_{n}\right\}$ in the following way:

$$
\begin{gathered}
y_{n}=\alpha_{n} x_{n}+\left(1-\alpha_{n}\right) T x_{n}, \\
x_{n+1}=\beta_{n} f\left(x_{n}\right)+\left(1-\beta_{n}\right) y_{n}, \quad \forall n \geq 0,
\end{gathered}
$$

where $\left\{\alpha_{n}\right\}$ and $\left\{\beta_{n}\right\}$ are two sequences in $(0,1)$.

They proved under certain control conditions on the sequences $\left\{\alpha_{n}\right\}$ and $\left\{\beta_{n}\right\}$ that $\left\{x_{n}\right\}$ converges strongly to a fixed point of $T$. Subsequently, under the following control conditions on $\left\{\alpha_{n}\right\}$ and $\left\{\beta_{n}\right\}$ :

(i) $1 \leq \beta_{n} \leq 1-\rho$, for all $n \geq n_{0}$ for some integer $n_{0} \geq 1$,

(ii) $\sum_{n=0}^{\infty} \beta_{n}=\infty$,

(iii) $0<\liminf _{n \rightarrow \infty} \alpha_{n} \leq \lim \sup _{n \rightarrow \infty} \alpha_{n}<1$,

(iv) $\lim _{n \rightarrow \infty}\left(\beta_{n+1} /\left(1-\left(1-\beta_{n+1}\right) \alpha_{n+1}\right)-\beta_{n} /(1-(1-\right.$ $\left.\left.\left.\beta_{n}\right) \alpha_{n}\right)\right)=0$.

Ceng and Yao [8] proved that

$$
x_{n} \longrightarrow q \Longleftrightarrow \beta_{n}\left(f\left(x_{n}\right)-x_{n}\right) \longrightarrow 0,
$$

where $q \in \operatorname{Fix}(T)$ solves the variational inequality problem (VIP):

$$
\langle q-f(q), J(q-p)\rangle \leq 0, \quad \forall p \in \operatorname{Fix}(T) .
$$

Such a result includes [7, Theorem 1] as a special case.

Let $C$ be a nonempty closed convex subset of a real Banach space $X$ and $f \in \Xi_{C}$ with a contractive coefficient $\rho \in(0,1)$, where $\Xi_{C}$ is the set of all contractive self-mappings on $C$. Let $\left\{T_{n}\right\}_{n=0}^{\infty}$ be a sequence of nonexpansive self-mappings on $C$ and $\left\{\lambda_{n}\right\}_{n=0}^{\infty}$ a sequence of nonnegative numbers in $[0,1]$. For any $n \geq 0$, define a self-mapping $W_{n}$ on $C$ as follows:

$$
\begin{aligned}
& U_{n, n+1}=I, \\
& U_{n, n}=\lambda_{n} T_{n} U_{n, n+1}+\left(1-\lambda_{n}\right) I, \\
& U_{n, n-1}=\lambda_{n-1} T_{n-1} U_{n, n}+\left(1-\lambda_{n-1}\right) I, \\
& \vdots \\
& U_{n, k}=\lambda_{k} T_{k} U_{n, k+1}+\left(1-\lambda_{k}\right) I, \\
& U_{n, k-1}=\lambda_{k-1} T_{k-1} U_{n, k}+\left(1-\lambda_{k-1}\right) I, \\
& \vdots \\
& U_{n, 1}=\lambda_{1} T_{1} U_{n, 2}+\left(1-\lambda_{1}\right) I, \\
& W_{n}=U_{n, 0}=\lambda_{0} T_{0} U_{n, 1}+\left(1-\lambda_{0}\right) I .
\end{aligned}
$$

Such a mapping $W_{n}$ is called the $W$-mapping generated by $T_{n}, T_{n-1}, \ldots, T_{0}$, and $\lambda_{n}, \lambda_{n-1}, \ldots, \lambda_{0}$; see [9].

In 2008, Ceng and Yao [10] introduced and analyzed the following relaxed viscosity approximation method for finding a common fixed point of an infinite family of nonexpansive mappings in a strictly convex and reflexive Banach space with a uniformly Gateaux differentiable norm.

Theorem 1 (see [10]). Let $X$ be a strictly convex and reflexive Banach space with a uniformly Gateaux differentiable norm, $C$ a nonempty closed convex subset of $X,\left\{T_{n}\right\}_{n=0}^{\infty}$ a sequence of nonexpansive self-mappings on $C$ such that the common fixed point set $F:=\bigcap_{n=0}^{\infty} \operatorname{Fix}\left(T_{n}\right) \neq \emptyset$, and $f \in \Xi_{C}$ with a contractive 
coefficient $\rho \in(1 / 2,1)$. For any given $x_{0} \in C$, let $\left\{x_{n}\right\}_{n=0}^{\infty}$ be the iterative sequence defined by

$$
\begin{gathered}
y_{n}=\left(1-\gamma_{n}\right) x_{n}+\gamma_{n} W_{n} x_{n} \\
x_{n+1}=\left(1-\alpha_{n}-\beta_{n}\right) x_{n}+\alpha_{n} f\left(y_{n}\right)+\beta_{n} W_{n} y_{n}, \quad \forall n \geq 0,
\end{gathered}
$$

where $\left\{\alpha_{n}\right\}_{n=0}^{\infty}$ and $\left\{\beta_{n}\right\}_{n=0}^{\infty}$ are two sequences in $(0,1)$ with $\alpha_{n}+$ $\beta_{n} \leq 1(n \geq 0),\left\{\gamma_{n}\right\}_{n=0}^{\infty}$ is a sequence in $[0,1]$, and $W_{n}$ is the $W$-mapping generated by (CY). Assume that

(i) $\lim _{n \rightarrow \infty} \alpha_{n}=0, \sum_{n=0}^{\infty} \alpha_{n}=\infty$ and $0<$ $\liminf _{n \rightarrow \infty} \beta_{n} \leq \limsup _{n \rightarrow \infty} \beta_{n}<1 ;$

(ii) $\lim _{n \rightarrow \infty}\left|\gamma_{n}-\gamma_{n-1}\right|=0$ and $\lim \sup _{n \rightarrow \infty} \gamma_{n}<1$.

Then, there hold the following:

(i) $\lim _{n \rightarrow \infty}\left\|x_{n+1}-x_{n}\right\|=0$;

(ii) the sequence $\left\{x_{n}\right\}_{n=0}^{\infty}$ converges strongly to some $p \in$ $F$ which is the unique solution of the variational inequality problem (VIP)

$$
\langle(I-f) q, J(q-p)\rangle \leq 0, \quad \forall f \in \Xi_{C}, p \in F,
$$

provided $\lim _{n \rightarrow \infty} \gamma_{n}=0$ and $\beta_{n} \equiv \beta$ for some fixed $\beta \in(0,1)$.

On the other hand, Cai and $\mathrm{Bu}$ [11] considered the following general system of variational inequalities (GSVI) in a real smooth Banach space $X$, which involves finding $\left(x^{*}, y^{*}\right) \in C \times C$ such that

$$
\begin{array}{ll}
\left\langle\mu_{1} B_{1} y^{*}+x^{*}-y^{*}, J\left(x-x^{*}\right)\right\rangle \geq 0, & \forall x \in C, \\
\left\langle\mu_{2} B_{2} x^{*}+y^{*}-x^{*}, J\left(x-y^{*}\right)\right\rangle \geq 0, & \forall x \in C,
\end{array}
$$

where $C$ is a nonempty, closed, and convex subset of $X$, $B_{1}, B_{2}: C \rightarrow X$ are two nonlinear mappings, and $\mu_{1}$ and $\mu_{2}$ are two positive constants. Here, the set of solutions of GSVI (13) is denoted by $\operatorname{GSVI}\left(C, B_{1}, B_{2}\right)$. In particular, if $X=H$, a real Hilbert space, then GSVI (13) reduces to the following GSVI of finding $\left(x^{*}, y^{*}\right) \in C \times C$ such that

$$
\begin{array}{ll}
\left\langle\mu_{1} B_{1} y^{*}+x^{*}-y^{*}, x-x^{*}\right\rangle \geq 0, & \forall x \in C, \\
\left\langle\mu_{2} B_{2} x^{*}+y^{*}-x^{*}, x-y^{*}\right\rangle \geq 0, & \forall x \in C,
\end{array}
$$

in which $\mu_{1}$ and $\mu_{2}$ are two positive constants. The set of solutions of problem (14) is still denoted by $\operatorname{GSVI}\left(C, B_{1}, B_{2}\right)$. In particular, if $B_{1}=B_{2}=A$, then problem (14) reduces to the new system of variational inequalities (NSVI), introduced and studied by Verma [12]. Further, if $x^{*}=y^{*}$ additionally, then the NSVI reduces to the classical variational inequality problem (VIP) of finding $x^{*} \in C$ such that

$$
\left\langle A x^{*}, x-x^{*}\right\rangle \geq 0, \quad \forall x \in C .
$$

The solution set of the $\operatorname{VIP}(15)$ is denoted by $\operatorname{VI}(C, A)$. Variational inequality theory has been studied quite extensively and has emerged as an important tool in the study of a wide class of obstacle, unilateral, free, moving, equilibrium problems. It is now well known that the variational inequalities are equivalent to the fixed point problems, the origin of which can be traced back to Lions and Stampacchia [13]. This alternative formulation has been used to suggest and analyze projection iterative method for solving variational inequalities under the conditions that the involved operator must be strongly monotone and Lipschitz continuous.

Recently, Ceng et al. [14] transformed problem (14) into a fixed point problem in the following way.

Lemma 2 (see [14]). For given $\bar{x}, \bar{y} \in C,(\bar{x}, \bar{y})$ is a solution of problem (14) if and only if $\bar{x}$ is a fixed point of the mapping $G: C \rightarrow C$ defined by

$$
\begin{array}{r}
G(x)=P_{C}\left[P_{C}\left(x-\mu_{2} B_{2} x\right)-\mu_{1} B_{1} P_{C}\left(x-\mu_{2} B_{2} x\right)\right], \\
\forall x \in C,
\end{array}
$$

where $\bar{y}=P_{C}\left(\bar{x}-\mu_{2} B_{2} \bar{x}\right)$ and $P_{C}$ is the projection of $H$ onto $C$.

In particular, if the mapping $B_{i}: C \rightarrow H$ is $\beta_{i}$-inverse strongly monotone for $i=1,2$, then the mapping $G$ is nonexpansive provided $\mu_{i} \in\left(0,2 \beta_{i}\right)$ for $i=1,2$.

In 1976, Korpelevič [15] proposed an iterative algorithm for solving the VIP (15) in Euclidean space $\mathbf{R}^{n}$ :

$$
\begin{gathered}
y_{n}=P_{C}\left(x_{n}-\tau A x_{n}\right), \\
x_{n+1}=P_{C}\left(x_{n}-\tau A y_{n}\right), \quad n \geq 0
\end{gathered}
$$

with $\tau>0$ a given number, which is known as the extragradient method (see also [16]). The literature on the VIP is vast and Korpelevich's extragradient method has received great attention given by many authors, who improved it in various ways; see, for example, $[3,11,13,17-33]$ and references therein, to name but a few.

In particular, whenever $X$ is still a real smooth Banach space, $B_{1}=B_{2}=A$ and $x^{*}=y^{*}$, then GSVI (13) reduces to the variational inequality problem (VIP) of finding $x^{*} \in C$ such that

$$
\left\langle A x^{*}, J\left(x-x^{*}\right)\right\rangle \geq 0, \quad \forall x \in C,
$$

which was considered by Aoyama et al. [34]. Note that VIP (18) is connected with the fixed point problem for nonlinear mapping (see, e.g., [35]), the problem of finding a zero point of a nonlinear operator (see, e.g., [36]), and so on. It is clear that VIP (18) extends VIP (15) from Hilbert spaces to Banach spaces.

In order to find a solution of VIP (18), Aoyama et al. [34] introduced the following Mann-type iterative scheme for an accretive operator $A$ :

$$
x_{n+1}=\alpha_{n} x_{n}+\left(1-\alpha_{n}\right) \Pi_{C}\left(x_{n}-\lambda_{n} A x_{n}\right), \quad \forall n \geq 1,
$$

where $\Pi_{C}$ is a sunny nonexpansive retraction from $X$ onto $C$. Then, they proved a weak convergence theorem. For the related work, see [37] and the references therein.

Let $C$ be a nonempty convex subset of a real Banach space $X$. Let $\left\{T_{i}\right\}_{i=1}^{N}$ be a finite family of nonexpansive mappings of 
$C$ into itself and let $\lambda_{1}, \ldots, \lambda_{N}$ be real numbers such that $0 \leq$ $\lambda_{i} \leq 1$ for every $i=1, \ldots, N$. Define a mapping $K: C \rightarrow C$ as follows:

$$
\begin{aligned}
& U_{1}=\lambda_{1} T_{1}+\left(1-\lambda_{1}\right) I, \\
& U_{2}=\lambda_{2} T_{2} U_{1}+\left(1-\lambda_{2}\right) U_{1}, \\
& U_{3}=\lambda_{3} T_{3} U_{2}+\left(1-\lambda_{3}\right) U_{2}, \\
& \vdots \\
& U_{N-1}=\lambda_{N-1} T_{N-1} U_{N-2}+\left(1-\lambda_{N-1}\right) U_{N-2}, \\
& K=U_{N}=\lambda_{N} T_{N} U_{N-1}+\left(1-\lambda_{N}\right) U_{N-1} .
\end{aligned}
$$

Such a mapping $K$ is called the $K$-mapping generated by $T_{1}, \ldots, T_{N}$ and $\lambda_{1}, \ldots, \lambda_{N}$.

Very recently, Kangtunyakarn [38] introduced and analyzed an iterative algorithm by the modification of Mann's iteration process for finding a common element of the set of solutions of a finite family of variational inequalities and the set of fixed points of an $\eta$-strictly pseudocontractive mapping and a nonexpansive mapping in uniformly convex and 2uniformly smooth Banach spaces.

Theorem 3 (see [38]). Let $C$ be a nonempty closed convex subset of a uniformly convex and 2-uniformly smooth Banach space $X$. Let $\Pi_{C}$ be a sunny nonexpansive retraction from $X$ onto C. Let $A_{i}: C \rightarrow X$ be an $\alpha_{i}$-inverse-strongly accretive mapping for each $i=1, \ldots, N$. Define the mapping $G_{i}$ : $C \rightarrow C$ by $G_{i}=\Pi_{C}\left(I-\lambda_{i} A_{i}\right)$ for $i=1, \ldots, N$, where $\lambda_{i} \in\left(0, \alpha_{i} / \kappa^{2}\right)$ and $\kappa$ is the 2-uniformly smooth constant of $X$. Let $B: C \rightarrow C$ be the K-mapping generated by $G_{1}, \ldots, G_{N}$ and $\rho_{1}, \ldots, \rho_{N}$, where $\rho_{i} \in(0,1)$, for all $i=1, \ldots, N-1$, and $\rho_{N} \in(0,1]$. Let $f: C \rightarrow C$ a contraction with coefficient $\rho \in(0,1)$. Let $V: C \rightarrow C$ be an $\eta$-strictly pseudocontractive mapping and $S: C \rightarrow C$ be a nonexpansive mapping such that $F=\operatorname{Fix}(S) \cap \operatorname{Fix}(V) \cap\left(\bigcap_{i=1}^{N} \operatorname{VI}\left(C, A_{i}\right)\right) \neq \emptyset$. For arbitrarily given $x_{0} \in C$, let $\left\{x_{n}\right\}$ be the sequence generated by

$$
\begin{array}{r}
x_{n+1}=\alpha_{n} f\left(x_{n}\right)+\beta_{n} x_{n}+\gamma_{n} B x_{n}+\delta_{n} S((1-\alpha) I+\alpha V) x_{n}, \\
\forall n \geq 0,
\end{array}
$$

where $\alpha \in\left(0, \eta / \kappa^{2}\right)$. Suppose that $\left\{\alpha_{n}\right\},\left\{\beta_{n}\right\},\left\{\gamma_{n}\right\}$, and $\left\{\delta_{n}\right\}$ are the sequences in $[0,1], \alpha_{n}+\beta_{n}+\gamma_{n}+\delta_{n}=1$ and satisfy the following conditions:

(i) $\lim _{n \rightarrow \infty} \alpha_{n}=0$ and $\sum_{n=0}^{\infty} \alpha_{n}=\infty$;

(ii) $\left\{\gamma_{n}\right\},\left\{\delta_{n}\right\} \subset[c, d]$ for some $c, d \in(0,1)$;

(iii) $\sum_{n=1}^{\infty}\left(\left|\beta_{n}-\beta_{n-1}\right|+\left|\gamma_{n}-\gamma_{n-1}\right|+\left|\delta_{n}-\delta_{n-1}\right|\right)<\infty$;

(iv) $0<\liminf _{n \rightarrow \infty} \beta_{n} \leq \lim \sup _{n \rightarrow \infty} \beta_{n}<1$.

Then, $\left\{x_{n}\right\}$ converges strongly to $q \in F$, which solves the following VIP:

$$
\langle q-f(q), J(q-p)\rangle \leq 0, \quad \forall p \in F .
$$

Beyond doubt, it is an interesting and valuable problem of constructing some algorithms with strong convergence for solving GSVI (13) which contains VIP (18) as a special case. Very recently, Cai and $\mathrm{Bu}$ [11] constructed an iterative algorithm for solving GSVI (13) and a common fixed point problem of a countable family of nonexpansive mappings in a uniformly convex and 2-uniformly smooth Banach space. They proved the strong convergence of the proposed algorithm by virtue of the following inequality in a 2uniformly smooth Banach space $X$.

Lemma 4 (see [39]). Let $X$ be a 2-uniformly smooth Banach space. Then,

$$
\|x+y\|^{2} \leq\|x\|^{2}+2\langle y, J(x)\rangle+2\|\kappa y\|^{2}, \quad \forall x, y \in X,
$$

where $\kappa$ is the 2-uniformly smooth constant of $X$ and $J$ is the normalized duality mapping from $X$ into $X^{*}$.

$$
\begin{aligned}
& \text { Define the mapping } G: C \rightarrow C \text { as follows: } \\
& G(x):=\Pi_{C}\left(I-\mu_{1} B_{1}\right) \Pi_{C}\left(I-\mu_{2} B_{2}\right) x, \quad \forall x \in C .
\end{aligned}
$$

The fixed point set of $G$ is denoted by $\Omega$. Then, their strong convergence theorem on the proposed method is stated as follows.

Theorem 5 (see [11]). Let $C$ be a nonempty closed convex subset of a uniformly convex and 2-uniformly smooth Banach space $X$. Let $\Pi_{C}$ be a sunny nonexpansive retraction from $X$ onto $C$. Let the mapping $B_{i}: C \rightarrow X$ be $\beta_{i}$-inverse-strongly accretive with $0<\mu_{i}<\beta_{i} / \kappa^{2}$ for $i=1,2$. Let $f$ be a contraction of $C$ into itself with coefficient $\delta \in(0,1)$. Let $\left\{T_{n}\right\}_{n=1}^{\infty}$ be a countable family of nonexpansive mappings of $C$ into itself such that $F=\bigcap_{i=1}^{\infty} \operatorname{Fix}\left(T_{i}\right) \cap \Omega \neq \emptyset$, where $\Omega$ is the fixed point set of the mapping $G$ defined by (24). For arbitrarily given $x_{1} \in C$, let $\left\{x_{n}\right\}$ be the sequence generated by

$$
\begin{gathered}
x_{n+1}=\beta_{n} x_{n}+\left(1-\beta_{n}\right) S_{n} y_{n}, \\
y_{n}=\alpha_{n} f\left(x_{n}\right)+\left(1-\alpha_{n}\right) z_{n}, \\
z_{n}=\Pi_{C}\left(u_{n}-\mu_{1} B_{1} u_{n}\right), \\
u_{n}=\Pi_{C}\left(x_{n}-\mu_{2} B_{2} x_{n}\right), \quad \forall n \geq 1 .
\end{gathered}
$$

Suppose that $\left\{\alpha_{n}\right\}$ and $\left\{\beta_{n}\right\}$ are two sequences in $(0,1)$ satisfying the following conditions:

(i) $\lim _{n \rightarrow \infty} \alpha_{n}=0$ and $\sum_{n=1}^{\infty} \alpha_{n}=\infty$;

(ii) $0<\liminf _{n \rightarrow \infty} \beta_{n} \leq \lim \sup _{n \rightarrow \infty} \beta_{n}<1$.

Assume that $\sum_{n=1}^{\infty} \sup _{x \in D}\left\|T_{n+1} x-T_{n} x\right\|<\infty$ for any bounded subset $D$ of $C$ and let $T$ be a mapping of $C$ into $X$ defined by $T x=\lim _{n \rightarrow \infty} T_{n} x$ for all $x \in C$ and suppose that $\operatorname{Fix}(T)=$ $\bigcap_{n=1}^{\infty} \operatorname{Fix}\left(T_{n}\right)$. Then, $\left\{x_{n}\right\}$ converges strongly to $q \in F$, which solves the following VIP:

$$
\langle q-f(q), J(q-p)\rangle \leq 0, \quad \forall p \in F .
$$


It is easy to see that the iterative scheme in Theorem 5 is essentially equivalent to the following two-step iterative scheme:

$$
\begin{gathered}
y_{n}=\alpha_{n} f\left(x_{n}\right)+\left(1-\alpha_{n}\right) G x_{n}, \\
x_{n+1}=\beta_{n} x_{n}+\left(1-\beta_{n}\right) T_{n} y_{n}, \quad \forall n \geq 1 .
\end{gathered}
$$

For the convenience of implementing the argument techniques in [14], the authors of [11] have used the following inequality in a real smooth and uniform convex Banach space $X$.

Proposition 6 (see [40]). Let $X$ be a real smooth and uniform convex Banach space and let $r>0$. Then, there exists a strictly increasing, continuous, and convex function $g:[0,2 r] \rightarrow \mathbf{R}$, $g(0)=0$ such that

$$
g(\|x-y\|) \leq\|x\|^{2}-2\langle x, J(y)\rangle+\|y\|^{2}, \quad \forall x, y \in B_{r},
$$

where $B_{r}=\{x \in X:\|x\| \leq r\}$.

Let $C$ be a nonempty closed convex subset of a real smooth Banach space $X$. Let $\Pi_{C}$ be a sunny nonexpansive retraction from $X$ onto $C$ and $f: C \rightarrow C$ a contraction with coefficient $\rho \in(0,1)$. Motivated and inspired by the research going on this area, we consider and introduce hybrid and relaxed Mann iteration methods for finding solutions of the GSVI (13) which are also common solutions of a countable family of variational inequalities and common fixed points of a countable family of nonexpansive mappings in $X$. Here, the hybrid and relaxed Mann iteration methods are based on Korpelevich's extragradient method, viscosity approximation method, and Mann iteration method. Under suitable assumptions, we derive some strong convergence theorems for hybrid and relaxed Mann iteration algorithms not only in the setting of uniformly convex and 2-uniformly smooth Banach space but also in a uniformly convex Banach space having a uniformly Gateaux differentiable norm. The results presented in this paper improve, extend, supplement, and develop the corresponding results announced in the earlier and very recent literature; see, for example, $[8,10,11$, $14,33,38]$.

\section{Preliminaries}

We list some lemmas that will be used in the sequel.

Lemma 7 (see [41]). Let $\left\{s_{n}\right\}$ be a sequence of nonnegative real numbers satisfying

$$
s_{n+1} \leq\left(1-\alpha_{n}\right) s_{n}+\alpha_{n} \beta_{n}+\gamma_{n}, \quad \forall n \geq 0,
$$

where $\left\{\alpha_{n}\right\},\left\{\beta_{n}\right\}$, and $\left\{\gamma_{n}\right\}$ satisfy the following conditions:

(i) $\left\{\alpha_{n}\right\} \subset[0,1]$ and $\sum_{n=0}^{\infty} \alpha_{n}=\infty$;

(ii) $\limsup _{n \rightarrow \infty} \beta_{n} \leq 0$;

(iii) $\gamma_{n} \geq 0$, for all $n \geq 0$, and $\sum_{n=0}^{\infty} \gamma_{n}<\infty$.

Then, $\lim \sup _{n \rightarrow \infty} s_{n}=0$.
The following lemma is an immediate consequence of the subdifferential inequality of the function $(1 / 2)\|\cdot\|^{2}$.

Lemma 8 (see [42]). Let $X$ be a real Banach space $X$. Then, for all $x, y \in X$

(i) $\|x+y\|^{2} \leq\|x\|^{2}+2\langle y, j(x+y)\rangle$ for all $j(x+y) \epsilon$ $J(x+y)$;

(ii) $\|x+y\|^{2} \geq\|x\|^{2}+2\langle y, j(x)\rangle$ for all $j(x) \in J(x)$.

Let $D$ be a subset of $C$ and let $\Pi$ be a mapping of $C$ into $D$. Then, $\Pi$ is said to be sunny if

$$
\Pi[\Pi(x)+t(x-\Pi(x))]=\Pi(x),
$$

whenever $\Pi(x)+t(x-\Pi(x)) \in C$ for $x \in C$ and $t \geq 0$. A mapping $\Pi$ of $C$ into itself is called a retraction if $\Pi^{2}=\Pi$. If a mapping $\Pi$ of $C$ into itself is a retraction, then $\Pi(z)=z$ for every $z \in R(\Pi)$ where $R(\Pi)$ is the range of $\Pi$. A subset $D$ of $C$ is called a sunny nonexpansive retract of $C$ if there exists a sunny nonexpansive retraction from $C$ onto $D$. The following lemma concerns the sunny nonexpansive retraction.

Lemma 9 (see [43]). Let $C$ be a nonempty closed convex subset of a real smooth Banach space $X$. Let $D$ be a nonempty subset of $C$. Let $\Pi$ be a retraction of $C$ onto $D$. Then, the following are equivalent:

(i) $\Pi$ is sunny and nonexpansive;

(ii) $\|\Pi(x)-\Pi(y)\|^{2} \leq\langle x-y, J(\Pi(x)-\Pi(y))\rangle$, for all $x, y \in C$;

(iii) $\langle x-\Pi(x), J(y-\Pi(x))\rangle \leq 0$, for all $x \in C, y \in D$.

It is well known that if $X=H$ a Hilbert space, then a sunny nonexpansive retraction $\Pi_{C}$ is coincident with the metric projection from $X$ onto $C$; that is, $\Pi_{C}=P_{C}$. If $C$ is a nonempty closed convex subset of a strictly convex and uniformly smooth Banach space $X$ and if $T: C \rightarrow C$ is a nonexpansive mapping with the fixed point set $\operatorname{Fix}(T) \neq \emptyset$, then the set $\operatorname{Fix}(T)$ is a sunny nonexpansive retract of $C$.

Lemma 10. Let $C$ be a nonempty closed convex subset of a smooth Banach space $X$. Let $\Pi_{C}$ be a sunny nonexpansive retraction from $X$ onto $C$ and let $B_{1}, B_{2}: C \rightarrow X$ be nonlinear mappings. For given $x^{*}, y^{*} \in C,\left(x^{*}, y^{*}\right)$ is a solution of GSVI (13) if and only if $x^{*}=\Pi_{C}\left(y^{*}-\mu_{1} B_{1} y^{*}\right)$, where $y^{*}=$ $\Pi_{C}\left(x^{*}-\mu_{2} B_{2} x^{*}\right)$.

Proof. We can rewrite GSVI (13) as

$$
\begin{aligned}
& \left\langle x^{*}-\left(y^{*}-\mu_{1} B_{1} y^{*}\right), J\left(x-x^{*}\right)\right\rangle \geq 0, \quad \forall x \in C, \\
& \left\langle y^{*}-\left(x^{*}-\mu_{2} B_{2} x^{*}\right), J\left(x-y^{*}\right)\right\rangle \geq 0, \quad \forall x \in C,
\end{aligned}
$$

which is obviously equivalent to

$$
\begin{aligned}
& x^{*}=\Pi_{C}\left(y^{*}-\mu_{1} B_{1} y^{*}\right), \\
& y^{*}=\Pi_{C}\left(x^{*}-\mu_{2} B_{2} x^{*}\right),
\end{aligned}
$$

because of Lemma 9. This completes the proof. 
In terms of Lemma 10, we observe that

$$
x^{*}=\Pi_{C}\left[\Pi_{C}\left(x^{*}-\mu_{2} B_{2} x^{*}\right)-\mu_{1} B_{1} \Pi_{C}\left(x^{*}-\mu_{2} B_{2} x^{*}\right)\right],
$$

which implies that $x^{*}$ is a fixed point of the mapping $G$. Throughout this paper, the set of fixed points of the mapping $G$ is denoted by $\Omega$.

Lemma 11 (see [44]). Let $X$ be a uniformly convex Banach space and $B_{r}=\{x \in X:\|x\| \leq r\}, r>0$. Then, there exists a continuous, strictly increasing, and convex function $g:[0, \infty] \rightarrow[0, \infty], g(0)=0$ such that

$$
\|\alpha x+\beta y+\gamma z\|^{2} \leq \alpha\|x\|^{2}+\beta\|y\|^{2}+\gamma\|z\|^{2}-\alpha \beta g(\|x-y\|)
$$

for all $x, y, z \in B_{r}$, and all $\alpha, \beta, \gamma \in[0,1]$ with $\alpha+\beta+\gamma=1$.

Lemma 12 (see [45]). Let $C$ be a nonempty closed convex subset of a Banach space $X$. Let $S_{0}, S_{1}, \ldots$ be a sequence of mappings of $C$ into itself. Suppose that $\sum_{n=1}^{\infty} \sup \left\{\left\|S_{n} x-S_{n-1} x\right\|\right.$ : $x \in C\}<\infty$. Then for each $y \in C,\left\{S_{n} y\right\}$ converges strongly to some point of $C$. Moreover, let $S$ be a mapping of $C$ into itself defined by $S y=\lim _{n \rightarrow \infty} S_{n} y$ for all $y \in C$. Then $\lim _{n \rightarrow \infty} \sup \left\{\left\|S x-S_{n} x\right\|: x \in C\right\}=0$.

Let $C$ be a nonempty closed convex subset of a Banach space $X$ and $T: C \rightarrow C$ a nonexpansive mapping with $\operatorname{Fix}(T) \neq \emptyset$. As previous, let $\Xi_{C}$ be the set of all contractions on $C$. For $t \in(0,1)$ and $f \in \Xi_{C}$, let $x_{t} \in C$ be the unique fixed point of the contraction $x \mapsto t f(x)+(1-t) T x$ on $C$; that is,

$$
x_{t}=t f\left(x_{t}\right)+(1-t) T x_{t} .
$$

Lemma 13 (see $[35,46])$. Let $X$ be a uniformly smooth Banach space, or a reflexive and strictly convex Banach space with a uniformly Gateaux differentiable norm. Let $C$ be a nonempty closed convex subset of $X, T: C \rightarrow C$ a nonexpansive mapping with $\operatorname{Fix}(T) \neq \emptyset$, and $f \in \Xi_{C}$. Then, the net $\left\{x_{t}\right\}$ defined by $x_{t}=$ $t f\left(x_{t}\right)+(1-t) T x_{t}$ converges strongly to a point in $\operatorname{Fix}(T)$. If we define a mapping $Q: \Xi_{C} \rightarrow \operatorname{Fix}(T)$ by $Q(f):=s-\lim _{t \rightarrow 0} x_{t}$, for all $f \in \Xi_{C}$, then $Q(f)$ solves the VIP:

$$
\langle(I-f) Q(f), J(Q(f)-p)\rangle \leq 0, \quad \forall f \in \Xi_{C}, p \in \operatorname{Fix}(T) .
$$

Lemma 14 (see [47]). Let $C$ be a nonempty closed convex subset of a strictly convex Banach space $X$. Let $\left\{T_{n}\right\}_{n=0}^{\infty}$ be a sequence of nonexpansive mappings on $C$. Suppose that $\bigcap_{n=0}^{\infty} \operatorname{Fix}\left(T_{n}\right)$ is nonempty. Let $\left\{\lambda_{n}\right\}$ be a sequence of positive numbers with $\sum_{n=0}^{\infty} \lambda_{n}=1$. Then, a mapping $S$ on $C$ defined by $S x=\sum_{n=0}^{\infty} \lambda_{n} T_{n} x$ for $x \in C$ is defined well; nonexpansive and $\operatorname{Fix}(S)=\bigcap_{n=0}^{\infty} \operatorname{Fix}\left(T_{n}\right)$ holds.

Lemma 15 (see [39]). Given a number $r>0$, A real Banach space $X$ is uniformly convex if and only if there exists a continuous strictly increasing function $g:[0, \infty) \rightarrow[0, \infty)$, $g(0)=0$, such that

$$
\begin{aligned}
\|\lambda x+(1-\lambda) y\|^{2} \leq & \lambda\|x\|^{2}+(1-\lambda)\|y\|^{2} \\
& -\lambda(1-\lambda) g(\|x-y\|)
\end{aligned}
$$

for all $\lambda \in[0,1]$ and $x, y \in X$ such that $\|x\| \leq r$ and $\|y\| \leq r$.

Lemma 16 (see [48, Lemma 3.2]). Let C be a nonempty closed convex subset of a strictly convex Banach space $X$. Let $\left\{T_{n}\right\}_{n=0}^{\infty}$ be a sequence of nonexpansive self-mappings on $C$ such that $\bigcap_{n=0}^{\infty} \operatorname{Fix}\left(T_{n}\right) \neq \emptyset$ and let $\left\{\lambda_{n}\right\}_{n=0}^{\infty}$ be a sequence of positive numbers in $(0, b]$ for some $b \in(0,1)$. Then, for every $x \in C$ and $k \geq 0$, the limit $\lim _{n \rightarrow \infty} U_{n, k} x$ exists.

Using Lemma 16, one can define a mapping $W: C \rightarrow C$ as follows:

$$
W x=\lim _{n \rightarrow \infty} W_{n} x=\lim _{n \rightarrow \infty} U_{n, 0} x
$$

for every $x \in C$. Such a $W$ is called the $W$-mapping generated by the sequences $\left\{T_{n}\right\}_{n=0}^{\infty}$ and $\left\{\lambda_{n}\right\}_{n=0}^{\infty}$. Throughout this paper, we always assume that $\left\{\lambda_{n}\right\}_{n=0}^{\infty}$ is a sequence of positive numbers in $(0, b]$ for some $b \in(0,1)$.

Lemma 17 (see [48]). Let $C$ be a nonempty closed convex subset of a strictly convex Banach space X. Let $\left\{T_{n}\right\}_{n=0}^{\infty}$ be a sequence of nonexpansive self-mappings on $C$ such that $\bigcap_{n=0}^{\infty} \operatorname{Fix}\left(T_{n}\right) \neq \emptyset$ and let $\left\{\lambda_{n}\right\}_{n=0}^{\infty}$ be a sequence of positive numbers in $(0, b]$ for some $b \in(0,1)$. Then, $\operatorname{Fix}(W)=$ $\bigcap_{n=0}^{\infty} \operatorname{Fix}\left(T_{n}\right)$.

Let $\mu$ be a continuous linear functional on $l^{\infty}$ and $s=$ $\left(a_{0}, a_{1}, \ldots\right) \in l^{\infty}$. One writes $\mu_{n}\left(a_{n}\right)$ instead of $\mu(s) . \mu$ is called a Banach limit if $\mu$ satisfies $\|\mu\|=\mu_{n}(1)=1$ and $\mu_{n}\left(a_{n+1}\right)=$ $\mu_{n}\left(a_{n}\right)$ for all $\left(a_{0}, a_{1}, \ldots\right) \in l^{\infty}$. If $\mu$ is a Banach limit, then, there hold the following:

(i) for all $n \geq 0, a_{n} \leq c_{n}$ implies $\mu_{n}\left(a_{n}\right) \leq \mu_{n}\left(c_{n}\right)$;

(ii) $\mu_{n}\left(a_{n+r}\right)=\mu_{n}\left(a_{n}\right)$ for any fixed positive integer $r$;

(iii) $\liminf _{n \rightarrow \infty} a_{n} \leq \mu_{n}\left(a_{n}\right) \leq \limsup _{n \rightarrow \infty} a_{n}$ for all $\left(a_{0}, a_{1}, \ldots\right) \in l^{\infty}$.

Lemma 18 (see [49]). Let $a \in \mathbf{R}$ be a real number and $a$ sequence $\left\{a_{n}\right\} \in l^{\infty}$ satisfy the condition $\mu_{n}\left(a_{n}\right) \leq$ a for all Banach limit $\mu$. If $\lim \sup _{n \rightarrow \infty}\left(a_{n+r}-a_{n}\right) \leq 0$, then $\lim \sup _{n \rightarrow \infty} a_{n} \leq a$.

In particular, if $r=1$ in Lemma 18 , then we immediately obtain the following corollary.

Corollary 19 (see [50]). Let $a \in \mathbf{R}$ be a real number and a sequence $\left\{a_{n}\right\} \in l^{\infty}$ satisfy the condition $\mu_{n}\left(a_{n}\right) \leq$ a for all Banach limit $\mu$. If $\lim \sup _{n \rightarrow \infty}\left(a_{n+1}-a_{n}\right) \leq 0$, then, $\lim \sup _{n \rightarrow \infty} a_{n} \leq a$.

Lemma 20 (see [51]). Let $\left\{x_{n}\right\}$ and $\left\{z_{n}\right\}$ be bounded sequences in a Banach space $X$ and let $\left\{\beta_{n}\right\}$ be a sequence of nonnegative numbers in $[0,1]$ with $0<\liminf _{n \rightarrow \infty} \beta_{n} \leq \lim \sup _{n \rightarrow \infty} \beta_{n}<$ 1. Suppose that $x_{n+1}=\beta_{n} x_{n}+\left(1-\beta_{n}\right) z_{n}$ for all integers 
$n \geq 0$ and $\lim \sup _{n \rightarrow \infty}\left(\left\|z_{n+1}-z_{n}\right\|-\left\|x_{n+1}-x_{n}\right\|\right) \leq 0$. Then, $\lim _{n \rightarrow \infty}\left\|x_{n}-z_{n}\right\|=0$.

Lemma 21 (see [34]). Let $C$ be a nonempty closed convex subset of a smooth Banach space $X$. Let $\Pi_{C}$ be a sunny nonexpansive retraction from $X$ onto $C$ and $A: C \rightarrow X$ an accretive mapping. Then for all $\lambda>0$,

$$
\operatorname{VI}(C, A)=\operatorname{Fix}\left(\Pi_{C}(I-\lambda A)\right)
$$

Lemma 22 (see [11]). Let $C$ be a nonempty closed convex subset of a real 2-uniformly smooth Banach space $X$. Let the mapping $B_{i}: C \rightarrow X$ be $\widehat{\beta}_{i}$-inverse-strongly accretive. Then, one has

$$
\begin{array}{r}
\left\|\left(I-\mu_{i} B_{i}\right) x-\left(I-\mu_{i} B_{i}\right) y\right\|^{2} \\
\leq\|x-y\|^{2}+2 \mu_{i}\left(\mu_{i} \kappa^{2}-\widehat{\beta}_{i}\right)\left\|B_{i} x-B_{i} y\right\|^{2}, \\
\forall x, y \in C,
\end{array}
$$

for $i=1,2$ where $\mu_{i}>0$. In particular, if $0<\mu_{i} \leq \widehat{\beta}_{i} / \kappa^{2}$, then $I-\mu_{i} B_{i}$ is nonexpansive for $i=1,2$.

Lemma 23 (see [11]). Let $C$ be a nonempty closed convex subset of a real 2-uniformly smooth Banach space $X$. Let $\Pi_{C}$ be a sunny nonexpansive retraction from $X$ onto $C$. Let the mapping $B_{i}: C \rightarrow X$ be $\widehat{\beta}_{i}$-inverse-strongly accretive for $i=1,2$. Let $G: C \rightarrow C$ be the mapping defined by

$$
\begin{array}{r}
G x=\Pi_{C}\left[\Pi_{C}\left(x-\mu_{2} B_{2} x\right)-\mu_{1} B_{1} \Pi_{C}\left(x-\mu_{2} B_{2} x\right)\right], \\
\forall x \in C .
\end{array}
$$

If $0<\mu_{i} \leq \widehat{\beta}_{i} / \kappa^{2}$ for $i=1,2$, then $G: C \rightarrow C$ is nonexpansive.

\section{Hybrid Mann Iterations and Their Convergence Criteria}

In this section, we introduce our hybrid Mann iteration algorithms in real smooth and uniformly convex Banach spaces and present their convergence criteria.

Theorem 24. Let $C$ be a nonempty closed convex subset of a uniformly convex and 2-uniformly smooth Banach space $X$. Let $\Pi_{C}$ be a sunny nonexpansive retraction from $X$ onto $C$. Let $\left\{\rho_{n}\right\}_{n=0}^{\infty}$ be a sequence of positive numbers in $(0, b]$ for some $b \in(0,1)$ and $A_{i}: C \rightarrow E$ an $\widehat{\alpha}_{i}$-inverse strongly accretive mapping for each $i=0,1, \ldots$. Define a mapping $G_{i}: C \rightarrow C$ by $\Pi_{C}\left(I-\lambda_{i} A_{i}\right) x=G_{i} x$ for all $x \in C$ and $i=0,1, \ldots$, where $\lambda_{i} \in\left(0, \widehat{\alpha}_{i} / \kappa^{2}\right], \kappa$ is the 2-uniformly smooth constant of $X$. Let $B_{n}: C \rightarrow C$ be the $W$-mapping generated by $G_{n}, G_{n-1}, \ldots, G_{0}$ and $\rho_{n}, \rho_{n-1}, \ldots, \rho_{0}$. Let the mapping $B_{i}: C \rightarrow X$ be $\widehat{\beta}_{i^{-}}$ inverse strongly accretive for $i=1,2$. Let $f: C \rightarrow C$ be a contraction with coefficient $\rho \in(0,1)$. Let $\left\{S_{i}\right\}_{i=0}^{\infty}$ be a countable family of nonexpansive mappings of $C$ into itself such that $F=$ $\left(\bigcap_{i=0}^{\infty} \operatorname{Fix}\left(S_{i}\right)\right) \cap \Omega \cap\left(\bigcap_{i=0}^{\infty} \operatorname{VI}\left(C, A_{i}\right)\right) \neq \emptyset$, where $\Omega$ is the fixed point set of the mapping $G=\Pi_{C}\left(I-\mu_{1} B_{1}\right) \Pi_{C}\left(I-\mu_{2} B_{2}\right)$ with $0<\mu_{i}<\widehat{\beta}_{i} / \kappa^{2}$ for $i=1,2$. For arbitrarily given $x_{0} \in C$, let $\left\{x_{n}\right\}$ be the sequence generated by

$$
\begin{gathered}
y_{n}=\beta_{n} x_{n}+\gamma_{n} B_{n} x_{n}+\delta_{n} S_{n} G x_{n}, \\
x_{n+1}=\alpha_{n} f\left(x_{n}\right)+\left(1-\alpha_{n}\right) y_{n}, \quad \forall n \geq 0,
\end{gathered}
$$

where $\left\{\alpha_{n}\right\},\left\{\beta_{n}\right\},\left\{\gamma_{n}\right\}$, and $\left\{\delta_{n}\right\}$ are the sequences in $[0,1]$ such that $\beta_{n}+\gamma_{n}+\delta_{n}=1$ for all $n \geq 0$. Suppose that the following conditions hold:

(i) $\sum_{n=0}^{\infty} \alpha_{n}=\infty$ and $0 \leq \alpha_{n} \leq 1-\rho$, for all $n \geq n_{0}$ for some integer $n_{0} \geq 0$;

(ii) $\liminf _{n \rightarrow \infty} \gamma_{n}>0$ and $\liminf _{n \rightarrow \infty} \delta_{n}>0$;

(iii) $\lim _{n \rightarrow \infty}\left(\mid \alpha_{n+1} /\left(1-\left(1-\alpha_{n+1}\right) \beta_{n+1}\right)-\alpha_{n} /(1-(1-\right.$ $\left.\left.\left.\alpha_{n}\right) \beta_{n}\right)|+| \delta_{n+1} /\left(1-\beta_{n+1}\right)-\delta_{n} /\left(1-\beta_{n}\right) \mid\right)=0 ;$

(iv) $0<\liminf _{n \rightarrow \infty} \beta_{n} \leq \limsup _{n \rightarrow \infty} \beta_{n}<1$.

Assume that $\sum_{n=0}^{\infty} \sup _{x \in D}\left\|S_{n+1} x-S_{n} x\right\|<\infty$ for any bounded subset $D$ of $C$ and let $S$ be a mapping of $C$ into itself defined by $S x=\lim _{n \rightarrow \infty} S_{n} x$ for all $x \in C$ and suppose that $\operatorname{Fix}(S)=\bigcap_{i=0}^{\infty} \operatorname{Fix}\left(S_{i}\right)$. Then, there hold the following:

(I) $\lim _{n \rightarrow \infty}\left\|x_{n+1}-x_{n}\right\|=0$;

(II) $x_{n} \rightarrow q \Leftrightarrow \alpha_{n}\left(f\left(x_{n}\right)-x_{n}\right) \rightarrow 0$ provided $\beta_{n} \equiv \beta$ for some fixed $\beta \in(0,1)$, where $q \in F$ solves the following VIP:

$$
\langle q-f(q), J(q-p)\rangle \leq 0, \quad \forall p \in F .
$$

Proof. First of all, since $0<\lambda_{i}<\widehat{\alpha}_{i} / \kappa^{2}$ for $i=0,1, \ldots$, it is easy to see that $G_{i}$ is a nonexpansive mapping for each $i=$ $0,1, \ldots$. Since $B_{n}: C \rightarrow C$ is the $W$-mapping generated by $G_{n}, G_{n-1}, \ldots, G_{0}$ and $\rho_{n}, \rho_{n-1}, \ldots, \rho_{0}$, by Lemma 16 we know that, for each $x \in C$ and $k \geq 0$, the limit $\lim _{n \rightarrow \infty} U_{n, k} x$ exists. Moreover, one can define a mapping $B: C \rightarrow C$ as follows:

$$
B x=\lim _{n \rightarrow \infty} B_{n} x=\lim _{n \rightarrow \infty} U_{n, 0} x
$$

for every $x \in C$. That is, such a $B$ is the $W$-mapping generated by the sequences $\left\{G_{n}\right\}_{n=0}^{\infty}$ and $\left\{\rho_{n}\right\}_{n=0}^{\infty}$. According to Lemma 17, we know that $\operatorname{Fix}(B)=\bigcap_{i=0}^{\infty} \operatorname{Fix}\left(G_{i}\right)$. From Lemma 15 and the definition of $G_{i}$, we have $\operatorname{Fix}\left(G_{i}\right)=\operatorname{VI}\left(C, A_{i}\right)$ for each $i=0,1, \ldots$. Hence, we have

$$
\operatorname{Fix}(B)=\bigcap_{i=0}^{\infty} \operatorname{Fix}\left(G_{i}\right)=\bigcap_{i=0}^{\infty} \operatorname{VI}\left(C, A_{i}\right) .
$$

Next, let us show that the sequence $\left\{x_{n}\right\}$ is bounded. Indeed, take a fixed $p \in F$ arbitrarily. Then, we get $p=G p$, $p=B_{n} p$, and $p=S_{n} p$ for all $n \geq 0$. By Lemma 23 we know that $G$ is nonexpansive. Then, from (42), we have

$$
\begin{aligned}
\left\|y_{n}-p\right\| & \leq \beta_{n}\left\|x_{n}-p\right\|+\gamma_{n}\left\|B_{n} x_{n}-p\right\|+\delta_{n}\left\|S_{n} G x_{n}-p\right\| \\
& \leq \beta_{n}\left\|x_{n}-p\right\|+\gamma_{n}\left\|x_{n}-p\right\|+\delta_{n}\left\|G x_{n}-p\right\| \\
& \leq \beta_{n}\left\|x_{n}-p\right\|+\gamma_{n}\left\|x_{n}-p\right\|+\delta_{n}\left\|x_{n}-p\right\| \\
& =\left\|x_{n}-p\right\|
\end{aligned}
$$


and hence

$$
\begin{aligned}
\left\|x_{n+1}-p\right\| \leq & \alpha_{n}\left\|f\left(x_{n}\right)-p\right\|+\left(1-\alpha_{n}\right)\left\|y_{n}-p\right\| \\
\leq & \alpha_{n}\left(\left\|f\left(x_{n}\right)-f(p)\right\|+\|f(p)-p\|\right) \\
& +\left(1-\alpha_{n}\right)\left\|y_{n}-p\right\| \\
\leq & \alpha_{n}\left(\rho\left\|x_{n}-p\right\|+\|f(p)-p\|\right) \\
& +\left(1-\alpha_{n}\right)\left\|x_{n}-p\right\| \\
= & \left(1-\alpha_{n}(1-\rho)\right)\left\|x_{n}-p\right\| \\
& +\alpha_{n}(1-\rho) \frac{\|f(p)-p\|}{1-\rho} \\
\leq & \max \left\{\left\|x_{n}-p\right\|, \frac{\|f(p)-p\|}{1-\rho}\right\} .
\end{aligned}
$$

By induction, we obtain

$$
\left\|x_{n}-p\right\| \leq \max \left\{\left\|x_{0}-p\right\|, \frac{\|f(p)-p\|}{1-\rho}\right\}, \quad \forall n \geq 0 .
$$

Thus, $\left\{x_{n}\right\}$ is bounded, and so are the sequences $\left\{y_{n}\right\}$, $\left\{G x_{n}\right\}$ and $\left\{f\left(x_{n}\right)\right\}$.

Let us show that

$$
\lim _{n \rightarrow \infty}\left\|x_{n+1}-x_{n}\right\|=0
$$

As a matter of fact, put $\sigma_{n}=\left(1-\alpha_{n}\right) \beta_{n}$, for all $n \geq 0$. Then, it follows from (i) and (iv) that

$$
\beta_{n} \geq \sigma_{n}=\left(1-\alpha_{n}\right) \beta_{n} \geq(1-(1-\rho)) \beta_{n}=\rho \beta_{n}, \quad \forall n \geq n_{0},
$$

and hence

$$
0<\liminf _{n \rightarrow \infty} \sigma_{n} \leq \limsup _{n \rightarrow \infty} \sigma_{n}<1
$$

Define

$$
x_{n+1}=\sigma_{n} x_{n}+\left(1-\sigma_{n}\right) z_{n} .
$$

Observe that

$$
\begin{aligned}
z_{n+1} & -z_{n} \\
= & \frac{x_{n+2}-\sigma_{n+1} x_{n+1}}{1-\sigma_{n+1}}-\frac{x_{n+1}-\sigma_{n} x_{n}}{1-\sigma_{n}} \\
= & \frac{\alpha_{n+1} f\left(x_{n+1}\right)+\left(1-\alpha_{n+1}\right) y_{n+1}-\sigma_{n+1} x_{n+1}}{1-\sigma_{n+1}} \\
& -\frac{\alpha_{n} f\left(x_{n}\right)+\left(1-\alpha_{n}\right) y_{n}-\sigma_{n} x_{n}}{1-\sigma_{n}}
\end{aligned}
$$

$$
\begin{aligned}
& =\left(\frac{\alpha_{n+1} f\left(x_{n+1}\right)}{1-\sigma_{n+1}}-\frac{\alpha_{n} f\left(x_{n}\right)}{1-\sigma_{n}}\right) \\
& -\frac{\left(1-\alpha_{n}\right)\left[\beta_{n} x_{n}+\gamma_{n} B_{n} x_{n}+\delta_{n} S_{n} G x_{n}\right]-\sigma_{n} x_{n}}{1-\sigma_{n}} \\
& +\left(1-\alpha_{n+1}\right)\left[\beta_{n+1} x_{n+1}+\gamma_{n+1} B_{n+1} x_{n+1}\right. \\
& \left.+\delta_{n+1} S_{n+1} G x_{n+1}\right] \\
& -\sigma_{n+1} x_{n+1} \times\left(1-\sigma_{n+1}\right)^{-1} \\
& =\left(\frac{\alpha_{n+1} f\left(x_{n+1}\right)}{1-\sigma_{n+1}}-\frac{\alpha_{n} f\left(x_{n}\right)}{1-\sigma_{n}}\right)+\frac{1-\alpha_{n+1}}{1-\sigma_{n+1}} \\
& \times\left(\gamma_{n+1} B_{n+1} x_{n+1}+\delta_{n+1} S_{n+1} G x_{n+1}\right) \\
& -\frac{1-\alpha_{n}}{1-\sigma_{n}}\left(\gamma_{n} B_{n} x_{n}+\delta_{n} S_{n} G x_{n}\right) \\
& =\left(\frac{\alpha_{n+1} f\left(x_{n+1}\right)}{1-\sigma_{n+1}}-\frac{\alpha_{n} f\left(x_{n}\right)}{1-\sigma_{n}}\right) \\
& +\frac{\left(1-\alpha_{n+1}\right)\left(1-\beta_{n+1}\right)}{1-\sigma_{n+1}} \\
& \times\left[\frac{\gamma_{n+1} B_{n+1} x_{n+1}+\delta_{n+1} S_{n+1} G x_{n+1}}{1-\beta_{n+1}}\right. \\
& \left.-\frac{\gamma_{n} B_{n} x_{n}+\delta_{n} S_{n} G x_{n}}{1-\beta_{n}}\right] \\
& +\left[\frac{\left(1-\alpha_{n+1}\right)\left(1-\beta_{n+1}\right)}{1-\sigma_{n+1}}-\frac{\left(1-\alpha_{n}\right)\left(1-\beta_{n}\right)}{1-\sigma_{n}}\right] \\
& \times \frac{\gamma_{n} B_{n} x_{n}+\delta_{n} S_{n} G x_{n}}{1-\beta_{n}}
\end{aligned}
$$$$
=\frac{\alpha_{n+1}}{1-\sigma_{n+1}}\left(f\left(x_{n+1}\right)-f\left(x_{n}\right)\right)
$$$$
+\left(\frac{\alpha_{n+1}}{1-\sigma_{n+1}}-\frac{\alpha_{n}}{1-\sigma_{n}}\right) f\left(x_{n}\right)
$$$$
+\frac{\left(1-\alpha_{n+1}\right)\left(1-\beta_{n+1}\right)}{1-\sigma_{n+1}}
$$$$
\times\left[\frac{\gamma_{n+1}}{\gamma_{n+1}+\delta_{n+1}}\left(B_{n+1} x_{n+1}-B_{n} x_{n}\right)\right.
$$$$
+\left(\frac{\gamma_{n+1}}{\gamma_{n+1}+\delta_{n+1}}-\frac{\gamma_{n}}{\gamma_{n}+\delta_{n}}\right) B_{n} x_{n}
$$$$
+\frac{\delta_{n+1}}{\gamma_{n+1}+\delta_{n+1}}\left(S_{n+1} G x_{n+1}-S_{n} G x_{n}\right)
$$$$
\left.+\left(\frac{\delta_{n+1}}{\gamma_{n+1}+\delta_{n+1}}-\frac{\delta_{n}}{\gamma_{n}+\delta_{n}}\right) S_{n} G x_{n}\right]
$$$$
-\left(\frac{\alpha_{n+1}}{1-\sigma_{n+1}}-\frac{\alpha_{n}}{1-\sigma_{n}}\right) \frac{\gamma_{n} B_{n} x_{n}+\delta_{n} S_{n} G x_{n}}{\gamma_{n}+\delta_{n}}
$$ 


$$
\begin{aligned}
& =\frac{\alpha_{n+1}}{1-\sigma_{n+1}}\left(f\left(x_{n+1}\right)-f\left(x_{n}\right)\right) \\
& +\left(\frac{\alpha_{n+1}}{1-\sigma_{n+1}}-\frac{\alpha_{n}}{1-\sigma_{n}}\right) \\
& \times\left(f\left(x_{n}\right)-\frac{\gamma_{n} B_{n} x_{n}+\delta_{n} S_{n} G x_{n}}{\gamma_{n}+\delta_{n}}\right) \\
& +\frac{1-\sigma_{n+1}-\alpha_{n+1}}{1-\sigma_{n+1}} \\
& \times\left[\frac{\gamma_{n+1}}{\gamma_{n+1}+\delta_{n+1}}\left(B_{n+1} x_{n+1}-B_{n} x_{n}\right)\right. \\
& \quad+\left(\frac{\gamma_{n+1}}{\gamma_{n+1}+\delta_{n+1}}-\frac{\gamma_{n}}{\gamma_{n}+\delta_{n}}\right) B_{n} x_{n} \\
& \quad+\frac{\delta_{n+1}}{\gamma_{n+1}+\delta_{n+1}}\left(S_{n+1} G x_{n+1}-S_{n} G x_{n}\right) \\
& \left.\quad+\left(\frac{\delta_{n+1}}{\gamma_{n+1}+\delta_{n+1}}-\frac{\delta_{n}}{\gamma_{n}+\delta_{n}}\right) S_{n} G x_{n}\right],
\end{aligned}
$$

and hence

$$
\begin{aligned}
& \left\|z_{n+1}-z_{n}\right\| \\
& \leq \frac{\alpha_{n+1}}{1-\sigma_{n+1}}\left\|f\left(x_{n+1}\right)-f\left(x_{n}\right)\right\| \\
& +\left|\frac{\alpha_{n+1}}{1-\sigma_{n+1}}-\frac{\alpha_{n}}{1-\sigma_{n}}\right|\left\|f\left(x_{n}\right)-\frac{\gamma_{n} B_{n} x_{n}+\delta_{n} S_{n} G x_{n}}{\gamma_{n}+\delta_{n}}\right\| \\
& +\frac{1-\sigma_{n+1}-\alpha_{n+1}}{1-\sigma_{n+1}} \\
& \times \| \frac{\gamma_{n+1}}{\gamma_{n+1}+\delta_{n+1}}\left(B_{n+1} x_{n+1}-B_{n} x_{n}\right) \\
& +\left(\frac{\gamma_{n+1}}{\gamma_{n+1}+\delta_{n+1}}-\frac{\gamma_{n}}{\gamma_{n}+\delta_{n}}\right) B_{n} x_{n} \\
& +\frac{\delta_{n+1}}{\gamma_{n+1}+\delta_{n+1}}\left(S_{n+1} G x_{n+1}-S_{n} G x_{n}\right) \\
& +\left(\frac{\delta_{n+1}}{\gamma_{n+1}+\delta_{n+1}}-\frac{\delta_{n}}{\gamma_{n}+\delta_{n}}\right) S_{n} G x_{n} \| \\
& \leq \frac{\rho \alpha_{n+1}}{1-\sigma_{n+1}}\left\|x_{n+1}-x_{n}\right\|+\left|\frac{\alpha_{n+1}}{1-\sigma_{n+1}}-\frac{\alpha_{n}}{1-\sigma_{n}}\right| \\
& \times\left(\left\|f\left(x_{n}\right)\right\|+\left\|B_{n} x_{n}\right\|+\left\|S_{n} G x_{n}\right\|\right) \\
& +\frac{1-\sigma_{n+1}-\alpha_{n+1}}{1-\sigma_{n+1}} \\
& \times\left[\frac{\gamma_{n+1}}{\gamma_{n+1}+\delta_{n+1}}\left\|B_{n+1} x_{n+1}-B_{n} x_{n}\right\|\right. \\
& +\left|\frac{\gamma_{n+1}}{\gamma_{n+1}+\delta_{n+1}}-\frac{\gamma_{n}}{\gamma_{n}+\delta_{n}}\right|\left\|B_{n} x_{n}\right\|
\end{aligned}
$$

$$
\begin{aligned}
& +\frac{\delta_{n+1}}{\gamma_{n+1}+\delta_{n+1}}\left\|S_{n+1} G x_{n+1}-S_{n} G x_{n}\right\| \\
& \left.+\left|\frac{\delta_{n+1}}{\gamma_{n+1}+\delta_{n+1}}-\frac{\delta_{n}}{\gamma_{n}+\delta_{n}}\right|\left\|S_{n} G x_{n}\right\|\right] .
\end{aligned}
$$

On the other hand, we note that, for all $n \geq 0$,

$$
\begin{aligned}
& \left\|S_{n+1} G x_{n+1}-S_{n} G x_{n}\right\| \\
& \quad \leq\left\|S_{n+1} G x_{n+1}-S_{n+1} G x_{n}\right\|+\left\|S_{n+1} G x_{n}-S_{n} G x_{n}\right\| \\
& \quad \leq\left\|G x_{n+1}-G x_{n}\right\|+\left\|S_{n+1} G x_{n}-S_{n} G x_{n}\right\| \\
& \quad \leq\left\|x_{n+1}-x_{n}\right\|+\left\|S_{n+1} G x_{n}-S_{n} G x_{n}\right\| .
\end{aligned}
$$

Furthermore, by (CY), since $G_{i}$ and $U_{n, i}$ are nonexpansive, we deduce that for each $n \geq 0$

$$
\begin{aligned}
& \left\|B_{n+1} x_{n+1}-B_{n} x_{n}\right\| \\
& \leq\left\|B_{n+1} x_{n+1}-B_{n+1} x_{n}\right\|+\left\|B_{n+1} x_{n}-B_{n} x_{n}\right\| \\
& \leq\left\|x_{n+1}-x_{n}\right\|+\left\|B_{n+1} x_{n}-B_{n} x_{n}\right\| \\
& \quad=\left\|x_{n+1}-x_{n}\right\|+\left\|\lambda_{0} G_{0} U_{n+1,1} x_{n}-\lambda_{0} G_{0} U_{n, 1} x_{n}\right\| \\
& \leq\left\|x_{n+1}-x_{n}\right\|+\lambda_{0}\left\|U_{n+1,1} x_{n}-U_{n, 1} x_{n}\right\| \\
& \quad=\left\|x_{n+1}-x_{n}\right\|+\lambda_{0}\left\|\lambda_{1} G_{1} U_{n+1,2} x_{n}-\lambda_{1} G_{1} U_{n, 2} x_{n}\right\| \\
& \leq\left\|x_{n+1}-x_{n}\right\|+\lambda_{0} \lambda_{1}\left\|U_{n+1,2} x_{n}-U_{n, 2} x_{n}\right\| \\
& \quad \quad \\
& \quad \leq\left\|x_{n+1}-x_{n}\right\|+\left(\prod_{i=0}^{n} \lambda_{i}\right)\left\|U_{n+1, n+1} x_{n}-U_{n, n+1} x_{n}\right\| \\
& \leq\left\|x_{n+1}-x_{n}\right\|+M_{0} \prod_{i=0}^{n} \lambda_{i},
\end{aligned}
$$

for some constant $M_{0}>0$. Utilizing (54)-(56), we have

$$
\begin{aligned}
& \left\|z_{n+1}-z_{n}\right\| \\
& \leq \frac{\rho \alpha_{n+1}}{1-\sigma_{n+1}}\left\|x_{n+1}-x_{n}\right\|+\left|\frac{\alpha_{n+1}}{1-\sigma_{n+1}}-\frac{\alpha_{n}}{1-\sigma_{n}}\right| \\
& \quad \times\left(\left\|f\left(x_{n}\right)\right\|+\left\|B_{n} x_{n}\right\|+\left\|S_{n} G x_{n}\right\|\right) \\
& \quad+\frac{1-\sigma_{n+1}-\alpha_{n+1}}{1-\sigma_{n+1}}
\end{aligned}
$$




$$
\begin{aligned}
& \times\left[\frac{\gamma_{n+1}}{\gamma_{n+1}+\delta_{n+1}}\left(\left\|x_{n+1}-x_{n}\right\|+M_{0} \prod_{i=0}^{n} \lambda_{i}\right)\right. \\
& +\left|\frac{\gamma_{n+1}}{\gamma_{n+1}+\delta_{n+1}}-\frac{\gamma_{n}}{\gamma_{n}+\delta_{n}}\right|\left\|B_{n} x_{n}\right\| \\
& +\frac{\delta_{n+1}}{\gamma_{n+1}+\delta_{n+1}}\left(\left\|x_{n+1}-x_{n}\right\|+\left\|S_{n+1} G x_{n}-S_{n} G x_{n}\right\|\right) \\
& \left.+\left|\frac{\delta_{n+1}}{\gamma_{n+1}+\delta_{n+1}}-\frac{\delta_{n}}{\gamma_{n}+\delta_{n}}\right|\left\|S_{n} G x_{n}\right\|\right] \\
& =\frac{1-\sigma_{n+1}-\alpha_{n+1}(1-\rho)}{1-\sigma_{n+1}}\left\|x_{n+1}-x_{n}\right\| \\
& +\left|\frac{\alpha_{n+1}}{1-\sigma_{n+1}}-\frac{\alpha_{n}}{1-\sigma_{n}}\right|\left(\left\|f\left(x_{n}\right)\right\|+\left\|B_{n} x_{n}\right\|+\left\|S_{n} G x_{n}\right\|\right) \\
& +\frac{1-\sigma_{n+1}-\alpha_{n+1}}{1-\sigma_{n+1}} \\
& \times\left[\frac{\gamma_{n+1}}{\gamma_{n+1}+\delta_{n+1}} M_{0} \prod_{i=0}^{n} \lambda_{i}\right. \\
& +\frac{\delta_{n+1}}{\gamma_{n+1}+\delta_{n+1}}\left\|S_{n+1} G x_{n}-S_{n} G x_{n}\right\| \\
& \left.+\left|\frac{\delta_{n+1}}{\gamma_{n+1}+\delta_{n+1}}-\frac{\delta_{n}}{\gamma_{n}+\delta_{n}}\right|\left(\left\|B_{n} x_{n}\right\|+\left\|S_{n} G x_{n}\right\|\right)\right] \\
& \leq\left\|x_{n+1}-x_{n}\right\|+\left|\frac{\alpha_{n+1}}{1-\sigma_{n+1}}-\frac{\alpha_{n}}{1-\sigma_{n}}\right| M \\
& +M \prod_{i=0}^{n} \lambda_{i}+\left\|S_{n+1} G x_{n}-S_{n} G x_{n}\right\| \\
& +\left|\frac{\delta_{n+1}}{\gamma_{n+1}+\delta_{n+1}}-\frac{\delta_{n}}{\gamma_{n}+\delta_{n}}\right| M \\
& =\left\|x_{n+1}-x_{n}\right\| \\
& +M\left(\left|\frac{\alpha_{n+1}}{1-\sigma_{n+1}}-\frac{\alpha_{n}}{1-\sigma_{n}}\right|\right. \\
& \left.+\left|\frac{\delta_{n+1}}{\gamma_{n+1}+\delta_{n+1}}-\frac{\delta_{n}}{\gamma_{n}+\delta_{n}}\right|+\prod_{i=0}^{n} \lambda_{i}\right) \\
& +\left\|S_{n+1} G x_{n}-S_{n} G x_{n}\right\| \text {, }
\end{aligned}
$$

which hence yields

$$
\begin{aligned}
& \left\|z_{n+1}-z_{n}\right\|-\left\|x_{n+1}-x_{n}\right\| \\
& \leq M\left(\left|\frac{\alpha_{n+1}}{1-\sigma_{n+1}}-\frac{\alpha_{n}}{1-\sigma_{n}}\right|+\left|\frac{\delta_{n+1}}{\gamma_{n+1}+\delta_{n+1}}-\frac{\delta_{n}}{\gamma_{n}+\delta_{n}}\right|\right. \\
& \left.\quad+\prod_{i=0}^{n} \lambda_{i}\right)+\left\|S_{n+1} G x_{n}-S_{n} G x_{n}\right\|,
\end{aligned}
$$

where $\sup _{n \geq 0}\left\{\left\|f\left(x_{n}\right)\right\|+\left\|B_{n} x_{n}\right\|+\left\|S_{n} G x_{n}\right\|+M_{0}\right\} \leq M$ for some $M>0$. So, from (58), condition (iii), and the assumption on $\left\{S_{n}\right\}$, it follows that (noting that $0<\lambda_{i} \leq b<1$, for all $i \geq 0$ )

$$
\limsup _{n \rightarrow \infty}\left(\left\|z_{n+1}-z_{n}\right\|-\left\|x_{n+1}-x_{n}\right\|\right) \leq 0 .
$$

Consequently, by Lemma 20, we have

$$
\lim _{n \rightarrow \infty}\left\|z_{n}-x_{n}\right\|=0 .
$$

It follows from (51) and (52) that

$$
\lim _{n \rightarrow \infty}\left\|x_{n+1}-x_{n}\right\|=\lim _{n \rightarrow \infty}\left(1-\sigma_{n}\right)\left\|z_{n}-x_{n}\right\|=0 .
$$

From (42), we have

$$
x_{n+1}-x_{n}=\alpha_{n}\left(f\left(x_{n}\right)-x_{n}\right)+\left(1-\alpha_{n}\right)\left(y_{n}-x_{n}\right) \text {, }
$$

which hence implies that

$$
\begin{aligned}
\rho\left\|y_{n}-x_{n}\right\| & =(1-(1-\rho))\left\|y_{n}-x_{n}\right\| \\
& \leq\left(1-\alpha_{n}\right)\left\|y_{n}-x_{n}\right\| \\
& =\left\|x_{n+1}-x_{n}-\alpha_{n}\left(f\left(x_{n}\right)-x_{n}\right)\right\| \\
& \leq\left\|x_{n+1}-x_{n}\right\|+\left\|\alpha_{n}\left(f\left(x_{n}\right)-x_{n}\right)\right\| .
\end{aligned}
$$

Since $x_{n+1}-x_{n} \rightarrow 0$ and $\alpha_{n}\left(f\left(x_{n}\right)-x_{n}\right) \rightarrow 0$, we get

$$
\lim _{n \rightarrow \infty}\left\|y_{n}-x_{n}\right\|=0 \text {. }
$$

Next, we show that $\left\|x_{n}-G x_{n}\right\| \rightarrow 0$ as $n \rightarrow \infty$.

Indeed, for simplicity, put $q=\Pi_{C}\left(p-\mu_{2} B_{2} p\right), u_{n}=$ $\Pi_{C}\left(x_{n}-\mu_{2} B_{2} x_{n}\right)$ and $v_{n}=\Pi_{C}\left(u_{n}-\mu_{1} B_{1} u_{n}\right)$. Then, $v_{n}=G x_{n}$ for all $n \geq 0$. From Lemma 22, we have

$$
\begin{aligned}
\left\|u_{n}-q\right\|^{2} & =\left\|\Pi_{C}\left(x_{n}-\mu_{2} B_{2} x_{n}\right)-\Pi_{C}\left(p-\mu_{2} B_{2} p\right)\right\|^{2} \\
& \leq\left\|x_{n}-p-\mu_{2}\left(B_{2} x_{n}-B_{2} p\right)\right\|^{2} \\
& \leq\left\|x_{n}-p\right\|^{2}-2 \mu_{2}\left(\widehat{\beta}_{2}-\kappa^{2} \mu_{2}\right)\left\|B_{2} x_{n}-B_{2} p\right\|^{2}, \\
\left\|v_{n}-p\right\|^{2} & =\left\|\Pi_{C}\left(u_{n}-\mu_{1} B_{1} u_{n}\right)-\Pi_{C}\left(q-\mu_{1} B_{1} q\right)\right\|^{2} \\
& \leq\left\|u_{n}-q-\mu_{1}\left(B_{1} u_{n}-B_{1} q\right)\right\|^{2} \\
& \leq\left\|u_{n}-q\right\|^{2}-2 \mu_{1}\left(\widehat{\beta}_{1}-\kappa^{2} \mu_{1}\right)\left\|B_{1} u_{n}-B_{1} q\right\|^{2} .
\end{aligned}
$$

Substituting (65) for (66), we obtain

$$
\begin{aligned}
\left\|v_{n}-p\right\|^{2} \leq & \left\|x_{n}-p\right\|^{2}-2 \mu_{2}\left(\widehat{\beta}_{2}-\kappa^{2} \mu_{2}\right)\left\|B_{2} x_{n}-B_{2} p\right\|^{2} \\
& -2 \mu_{1}\left(\widehat{\beta}_{1}-\kappa^{2} \mu_{1}\right)\left\|B_{1} u_{n}-B_{1} q\right\|^{2} .
\end{aligned}
$$


From (42) and (67), we have

$$
\begin{aligned}
& \left\|y_{n}-p\right\|^{2} \\
& \leq \beta_{n}\left\|x_{n}-p\right\|^{2}+\gamma_{n}\left\|B_{n} x_{n}-p\right\|^{2}+\delta_{n}\left\|S_{n} G x_{n}-p\right\|^{2} \\
& \leq \beta_{n}\left\|x_{n}-p\right\|^{2}+\gamma_{n}\left\|x_{n}-p\right\|^{2}+\delta_{n}\left\|v_{n}-p\right\|^{2} \\
& \leq \beta_{n}\left\|x_{n}-p\right\|^{2}+\gamma_{n}\left\|x_{n}-p\right\|^{2} \\
& +\delta_{n}\left[\left\|x_{n}-p\right\|^{2}-2 \mu_{2}\left(\widehat{\beta}_{2}-\kappa^{2} \mu_{2}\right)\right. \\
& \quad \times\left\|B_{2} x_{n}-B_{2} p\right\|^{2} \\
& \left.\quad-2 \mu_{1}\left(\widehat{\beta}_{1}-\kappa^{2} \mu_{1}\right)\left\|B_{1} u_{n}-B_{1} q\right\|^{2}\right] \\
& =\left\|x_{n}-p\right\|^{2} \\
& -2 \delta_{n}\left[\mu_{2}\left(\widehat{\beta}_{2}-\kappa^{2} \mu_{2}\right)\left\|B_{2} x_{n}-B_{2} p\right\|^{2}\right. \\
& \left.+2 \mu_{1}\left(\widehat{\beta}_{1}-\kappa^{2} \mu_{1}\right)\left\|B_{1} u_{n}-B_{1} q\right\|^{2}\right]
\end{aligned}
$$

which hence implies that

$$
\begin{aligned}
2 \delta_{n} & {\left[\mu_{2}\left(\widehat{\beta}_{2}-\kappa^{2} \mu_{2}\right)\left\|B_{2} x_{n}-B_{2} p\right\|^{2}\right.} \\
& \left.+\mu_{1}\left(\widehat{\beta}_{1}-\kappa^{2} \mu_{1}\right)\left\|B_{1} u_{n}-B_{1} q\right\|^{2}\right] \\
\leq & \left\|x_{n}-p\right\|^{2}-\left\|y_{n}-p\right\|^{2} \\
\leq & \left(\left\|x_{n}-p\right\|+\left\|y_{n}-p\right\|\right)\left\|x_{n}-y_{n}\right\| .
\end{aligned}
$$

Since $0<\mu_{i}<\widehat{\beta}_{i} / \kappa^{2}$ for $i=1,2$, and $\left\{x_{n}\right\},\left\{y_{n}\right\}$ are bounded, we obtain from (64), (69), and condition (ii) that

$$
\lim _{n \rightarrow \infty}\left\|B_{2} x_{n}-B_{2} p\right\|=0, \quad \lim _{n \rightarrow \infty}\left\|B_{1} u_{n}-B_{1} q\right\|=0 .
$$

Utilizing Proposition 6 and Lemma 9, we have

$$
\begin{aligned}
&\left\|u_{n}-q\right\|^{2} \\
&=\left\|\Pi_{C}\left(x_{n}-\mu_{2} B_{2} x_{n}\right)-\Pi_{C}\left(p-\mu_{2} B_{2} p\right)\right\|^{2} \\
& \leq\left\langle x_{n}-\mu_{2} B_{2} x_{n}-\left(p-\mu_{2} B_{2} p\right), J\left(u_{n}-q\right)\right\rangle \\
&=\left\langle x_{n}-p, J\left(u_{n}-q\right)\right\rangle+\mu_{2}\left\langle B_{2} p-B_{2} x_{n}, J\left(u_{n}-q\right)\right\rangle \\
& \leq \frac{1}{2}\left[\left\|x_{n}-p\right\|^{2}+\left\|u_{n}-q\right\|^{2}-g_{1}\left(\left\|x_{n}-u_{n}-(p-q)\right\|\right)\right] \\
&+\mu_{2}\left\|B_{2} p-B_{2} x_{n}\right\|\left\|u_{n}-q\right\|,
\end{aligned}
$$

which implies that

$$
\begin{gathered}
\left\|u_{n}-q\right\|^{2} \leq\left\|x_{n}-p\right\|^{2}-g_{1}\left(\left\|x_{n}-u_{n}-(p-q)\right\|\right) \\
+2 \mu_{2}\left\|B_{2} p-B_{2} x_{n}\right\|\left\|u_{n}-q\right\| .
\end{gathered}
$$

In the same way, we derive

$$
\begin{aligned}
\left\|v_{n}-p\right\|^{2} \\
=\left\|\Pi_{C}\left(u_{n}-\mu_{1} B_{1} u_{n}\right)-\Pi_{C}\left(q-\mu_{1} B_{1} q\right)\right\|^{2} \\
\leq\left\langle u_{n}-\mu_{1} B_{1} u_{n}-\left(q-\mu_{1} B_{1} q\right), J\left(v_{n}-p\right)\right\rangle \\
=\left\langle u_{n}-q, J\left(v_{n}-p\right)\right\rangle+\mu_{1}\left\langle B_{1} q-B_{1} u_{n}, J\left(v_{n}-p\right)\right\rangle \\
\leq \frac{1}{2}\left[\left\|u_{n}-q\right\|^{2}+\left\|v_{n}-p\right\|^{2}\right. \\
\left.\quad \quad-g_{2}\left(\left\|u_{n}-v_{n}+(p-q)\right\|\right)\right] \\
\quad+\mu_{1}\left\|B_{1} q-B_{1} u_{n}\right\|\left\|v_{n}-p\right\|,
\end{aligned}
$$

which implies that

$$
\begin{aligned}
\left\|v_{n}-p\right\|^{2} \leq & \left\|u_{n}-q\right\|^{2}-g_{2}\left(\left\|u_{n}-v_{n}+(p-q)\right\|\right) \\
& +2 \mu_{1}\left\|B_{1} q-B_{1} u_{n}\right\|\left\|v_{n}-p\right\|
\end{aligned}
$$

Substituting (72) for (74), we get

$$
\begin{aligned}
\| v_{n}- & p \|^{2} \\
\leq & \left\|x_{n}-p\right\|^{2}-g_{1}\left(\left\|x_{n}-u_{n}-(p-q)\right\|\right) \\
& -g_{2}\left(\left\|u_{n}-v_{n}+(p-q)\right\|\right) \\
& +2 \mu_{2}\left\|B_{2} p-B_{2} x_{n}\right\|\left\|u_{n}-q\right\| \\
& +2 \mu_{1}\left\|B_{1} q-B_{1} u_{n}\right\|\left\|v_{n}-p\right\| .
\end{aligned}
$$

By Lemma 8(i), we have from (68) and (75)

$$
\begin{aligned}
& \left\|y_{n}-p\right\|^{2} \\
& \leq \beta_{n}\left\|x_{n}-p\right\|^{2}+\gamma_{n}\left\|x_{n}-p\right\|^{2}+\delta_{n}\left\|v_{n}-p\right\|^{2} \\
& \leq \beta_{n}\left\|x_{n}-p\right\|^{2}+\gamma_{n}\left\|x_{n}-p\right\|^{2} \\
& +\delta_{n}\left[\left\|x_{n}-p\right\|^{2}-g_{1}\left(\left\|x_{n}-u_{n}-(p-q)\right\|\right)\right. \\
& \quad-g_{2}\left(\left\|u_{n}-v_{n}+(p-q)\right\|\right)+2 \mu_{2}\left\|B_{2} p-B_{2} x_{n}\right\| \\
& \left.\quad \times\left\|u_{n}-q\right\|+2 \mu_{1}\left\|B_{1} q-B_{1} u_{n}\right\|\left\|v_{n}-p\right\|\right] \\
& \leq\left\|x_{n}-p\right\|^{2} \\
& \quad-\delta_{n}\left[g_{1}\left(\left\|x_{n}-u_{n}-(p-q)\right\|\right)\right. \\
& \left.\quad+g_{2}\left(\left\|u_{n}-v_{n}+(p-q)\right\|\right)\right] \\
& +2 \mu_{2}\left\|B_{2} p-B_{2} x_{n}\right\|\left\|u_{n}-q\right\| \\
& +2 \mu_{1}\left\|B_{1} q-B_{1} u_{n}\right\|\left\|v_{n}-p\right\|,
\end{aligned}
$$


which hence leads to

$$
\begin{aligned}
& \delta_{n}\left[g_{1}\left(\left\|x_{n}-u_{n}-(p-q)\right\|\right)+g_{2}\left(\left\|u_{n}-v_{n}+(p-q)\right\|\right)\right] \\
& \leq\left\|x_{n}-p\right\|^{2}-\left\|y_{n}-p\right\|^{2}+2 \mu_{2}\left\|B_{2} p-B_{2} x_{n}\right\|\left\|u_{n}-q\right\| \\
& \quad+2 \mu_{1}\left\|B_{1} q-B_{1} u_{n}\right\|\left\|v_{n}-p\right\| \\
& \leq\left(\left\|x_{n}-p\right\|+\left\|y_{n}-p\right\|\right)\left\|x_{n}-y_{n}\right\| \\
& \quad+2 \mu_{2}\left\|B_{2} p-B_{2} x_{n}\right\|\left\|u_{n}-q\right\| \\
& \quad+2 \mu_{1}\left\|B_{1} q-B_{1} u_{n}\right\|\left\|v_{n}-p\right\| .
\end{aligned}
$$

From (70), (77), condition (ii), and the boundedness of $\left\{x_{n}\right\},\left\{y_{n}\right\},\left\{u_{n}\right\}$, and $\left\{v_{n}\right\}$, we deduce that

$$
\begin{aligned}
& \lim _{n \rightarrow \infty} g_{1}\left(\left\|x_{n}-u_{n}-(p-q)\right\|\right)=0, \\
& \lim _{n \rightarrow \infty} g_{2}\left(\left\|u_{n}-v_{n}+(p-q)\right\|\right)=0 .
\end{aligned}
$$

Utilizing the properties of $g_{1}$ and $g_{2}$, we deduce that

$$
\begin{aligned}
& \lim _{n \rightarrow \infty}\left\|x_{n}-u_{n}-(p-q)\right\|=0, \\
& \lim _{n \rightarrow \infty}\left\|u_{n}-v_{n}+(p-q)\right\|=0
\end{aligned}
$$

From (79), we get

$$
\begin{aligned}
\left\|x_{n}-v_{n}\right\| \leq & \left\|x_{n}-u_{n}-(p-q)\right\| \\
& +\left\|u_{n}-v_{n}+(p-q)\right\| \longrightarrow 0 \quad \text { as } n \longrightarrow \infty
\end{aligned}
$$

That is,

$$
\lim _{n \rightarrow \infty}\left\|x_{n}-G x_{n}\right\|=0
$$

Next, let us show that

$$
\lim _{n \rightarrow \infty}\left\|B_{n} x_{n}-x_{n}\right\|=0, \quad \lim _{n \rightarrow \infty}\left\|S x_{n}-x_{n}\right\|=0 .
$$

Indeed, utilizing Lemma 15 and (42), we have

$$
\begin{aligned}
& \left\|y_{n}-p\right\|^{2} \\
& =\left\|\delta_{n}\left(S_{n} G x_{n}-p\right)+\left(\beta_{n}+\gamma_{n}\right)\left(\frac{\beta_{n} x_{n}+\gamma_{n} B_{n} x_{n}}{\beta_{n}+\gamma_{n}}-p\right)\right\|^{2} \\
& \leq \delta_{n}\left\|S_{n} G x_{n}-p\right\|^{2}+\left(\beta_{n}+\gamma_{n}\right) \\
& \quad \times\left\|\frac{\beta_{n} x_{n}+\gamma_{n} B_{n} x_{n}}{\beta_{n}+\gamma_{n}}-p\right\|^{2}
\end{aligned}
$$

$$
\begin{aligned}
= & \delta_{n}\left\|S_{n} G x_{n}-p\right\|^{2}+\left(\beta_{n}+\gamma_{n}\right) \\
& \times\left\|\frac{\beta_{n}}{\beta_{n}+\gamma_{n}}\left(x_{n}-p\right)+\frac{\gamma_{n}}{\beta_{n}+\gamma_{n}}\left(B_{n} x_{n}-p\right)\right\|^{2} \\
\leq & \delta_{n}\left\|G x_{n}-p\right\|^{2}+\left(\beta_{n}+\gamma_{n}\right) \\
& \times\left[\frac{\beta_{n}}{\beta_{n}+\gamma_{n}}\left\|x_{n}-p\right\|^{2}+\frac{\gamma_{n}}{\beta_{n}+\gamma_{n}}\left\|B_{n} x_{n}-p\right\|^{2}\right. \\
& \left.\quad-\frac{\beta_{n} \gamma_{n}}{\left(\beta_{n}+\gamma_{n}\right)^{2}} g_{3}\left(\left\|x_{n}-B_{n} x_{n}\right\|\right)\right] \\
\leq & \delta_{n}\left\|x_{n}-p\right\|^{2}+\beta_{n}\left\|x_{n}-p\right\|^{2}+\gamma_{n}\left\|x_{n}-p\right\|^{2} \\
& -\frac{\beta_{n} \gamma_{n}}{\beta_{n}+\gamma_{n}} g_{3}\left(\left\|x_{n}-B_{n} x_{n}\right\|\right) \\
= & \left\|x_{n}-p\right\|^{2}-\frac{\beta_{n} \gamma_{n}}{\beta_{n}+\gamma_{n}} g_{3}\left(\left\|x_{n}-B_{n} x_{n}\right\|\right),
\end{aligned}
$$

which immediately implies that

$$
\begin{aligned}
\beta_{n} \gamma_{n} g_{3} & \left(\left\|x_{n}-B_{n} x_{n}\right\|\right) \\
& \leq \frac{\beta_{n} \gamma_{n}}{\beta_{n}+\gamma_{n}} g_{3}\left(\left\|x_{n}-B_{n} x_{n}\right\|\right) \\
& \leq\left\|x_{n}-p\right\|^{2}-\left\|y_{n}-p\right\|^{2} \\
& \leq\left(\left\|x_{n}-p\right\|+\left\|y_{n}-p\right\|\right)\left\|x_{n}-y_{n}\right\| .
\end{aligned}
$$

So, from (64), the boundedness of $\left\{x_{n}\right\},\left\{y_{n}\right\}$, and conditions (ii), (iv), it follows that

$$
\lim _{n \rightarrow \infty} g_{3}\left(\left\|x_{n}-B_{n} x_{n}\right\|\right)=0 .
$$

From the properties of $g_{3}$, we have

$$
\lim _{n \rightarrow \infty}\left\|x_{n}-B_{n} x_{n}\right\|=0
$$

Taking into account that

$$
y_{n}-x_{n}=\gamma_{n}\left(B_{n} x_{n}-x_{n}\right)+\delta_{n}\left(S_{n} G x_{n}-x_{n}\right),
$$

we have

$$
\begin{aligned}
\delta_{n}\left\|S_{n} G x_{n}-x_{n}\right\| & \\
& =\left\|y_{n}-x_{n}-\gamma_{n}\left(B_{n} x_{n}-x_{n}\right)\right\| \\
& \leq\left\|y_{n}-x_{n}\right\|+\gamma_{n}\left\|B_{n} x_{n}-x_{n}\right\| \\
& \leq\left\|y_{n}-x_{n}\right\|+\left\|B_{n} x_{n}-x_{n}\right\| .
\end{aligned}
$$

From (64), (86), and condition (ii), it follows that

$$
\lim _{n \rightarrow \infty}\left\|S_{n} G x_{n}-x_{n}\right\|=0, \quad \lim _{n \rightarrow \infty}\left\|S_{n} G x_{n}-B_{n} x_{n}\right\|=0 .
$$


Note that

$$
\begin{aligned}
\| x_{n}- & S x_{n} \| \\
\leq & \left\|x_{n}-S_{n} G x_{n}\right\|+\left\|S_{n} G x_{n}-S_{n} x_{n}\right\| \\
& +\left\|S_{n} x_{n}-S x_{n}\right\| \\
\leq & \left\|x_{n}-S_{n} G x_{n}\right\|+\left\|G x_{n}-x_{n}\right\| \\
& +\left\|S_{n} x_{n}-S x_{n}\right\| .
\end{aligned}
$$

So, in terms of (81), (89), and Lemma 12, we have

$$
\lim _{n \rightarrow \infty}\left\|x_{n}-S x_{n}\right\|=0 .
$$

Suppose that $\beta_{n} \equiv \beta$ for some fixed $\beta \in(0,1)$ such that $\beta+$ $\gamma_{n}+\delta_{n}=1$ for all $n \geq 0$. Define a mapping $V x=\left(1-\theta_{1}-\right.$ $\left.\theta_{2}\right) S x+\theta_{1} B x+\theta_{2} G x$, where $\theta_{1}, \theta_{2} \in(0,1)$ are two constants with $\theta_{1}+\theta_{2}<1$. Then, by Lemmas 14 and 17, we have that $\operatorname{Fix}(V)=\operatorname{Fix}(S) \cap \operatorname{Fix}(B) \cap \operatorname{Fix}(G)=F$. For each $k \geq 1$, let $\left\{p_{k}\right\}$ be a unique element of $C$ such that

$$
p_{k}=\frac{1}{k} f\left(p_{k}\right)+\left(1-\frac{1}{k}\right) V p_{k} .
$$

From Lemma 13, we conclude that $p_{k} \rightarrow q \in \operatorname{Fix}(V)=F$ as $k \rightarrow \infty$. Observe that for every $n, k$

$$
\begin{aligned}
\| y_{n}- & B p_{k} \| \\
= & \left\|\beta\left(x_{n}-B p_{k}\right)+\gamma_{n}\left(B_{n} x_{n}-B p_{k}\right)+\delta_{n}\left(S_{n} G x_{n}-B p_{k}\right)\right\| \\
\leq & \beta\left\|x_{n}-B p_{k}\right\|+\gamma_{n}\left\|B_{n} x_{n}-B p_{k}\right\| \\
& +\delta_{n}\left(\left\|S_{n} G x_{n}-B_{n} x_{n}\right\|+\left\|B_{n} x_{n}-B p_{k}\right\|\right) \\
= & \beta\left\|x_{n}-B p_{k}\right\|+(1-\beta)\left\|B_{n} x_{n}-B p_{k}\right\| \\
& +\delta_{n}\left\|S_{n} G x_{n}-B_{n} x_{n}\right\|,
\end{aligned}
$$

and hence

$$
\begin{aligned}
& \left\|x_{n+1}-B p_{k}\right\| \\
& \leq \alpha_{n}\left\|f\left(x_{n}\right)-B p_{k}\right\|+\left(1-\alpha_{n}\right)\left\|y_{n}-B p_{k}\right\| \\
& \leq \alpha_{n}\left(\left\|f\left(x_{n}\right)-x_{n}\right\|+\left\|x_{n}-B p_{k}\right\|\right) \\
& \quad+\left(1-\alpha_{n}\right)\left\|y_{n}-B p_{k}\right\| \\
& \leq \alpha_{n}\left\|f\left(x_{n}\right)-x_{n}\right\|+\alpha_{n}\left\|x_{n}-B p_{k}\right\|+\left(1-\alpha_{n}\right) \\
& \quad \times\left[\beta\left\|x_{n}-B p_{k}\right\|\right. \\
& \left.\quad+(1-\beta)\left\|B_{n} x_{n}-B p_{k}\right\|+\delta_{n}\left\|S_{n} G x_{n}-B_{n} x_{n}\right\|\right]
\end{aligned}
$$

$$
\begin{aligned}
& \leq \alpha_{n}\left\|f\left(x_{n}\right)-x_{n}\right\|+\alpha_{n}\left\|x_{n}-B p_{k}\right\|+\left(1-\alpha_{n}\right) \\
& \times\left[\beta\left\|x_{n}-B p_{k}\right\|\right. \\
& +(1-\beta)\left(\left\|B_{n} x_{n}-B_{n} p_{k}\right\|+\left\|B_{n} p_{k}-B p_{k}\right\|\right) \\
& \left.+\delta_{n}\left\|S_{n} G x_{n}-B_{n} x_{n}\right\|\right] \\
& \leq \alpha_{n}\left\|f\left(x_{n}\right)-x_{n}\right\|+\alpha_{n}\left\|x_{n}-B p_{k}\right\| \\
& +\left(1-\alpha_{n}\right)\left[\beta\left\|x_{n}-B p_{k}\right\|\right. \\
& +(1-\beta)\left(\left\|x_{n}-p_{k}\right\|+\left\|B_{n} p_{k}-B p_{k}\right\|\right) \\
& \left.+\delta_{n}\left\|S_{n} G x_{n}-B_{n} x_{n}\right\|\right] \\
& =\alpha_{n}\left\|f\left(x_{n}\right)-x_{n}\right\|+\left(\beta+\alpha_{n}(1-\beta)\right) \\
& \times\left\|x_{n}-B p_{k}\right\|+\left(1-\alpha_{n}\right)(1-\beta)\left\|x_{n}-p_{k}\right\| \\
& +\left(1-\alpha_{n}\right)\left[(1-\beta)\left\|B_{n} p_{k}-B p_{k}\right\|\right. \\
& \left.+\delta_{n}\left\|S_{n} G x_{n}-B_{n} x_{n}\right\|\right] \\
& \leq \alpha_{n}\left\|f\left(x_{n}\right)-x_{n}\right\|+\left(\beta+\alpha_{n}(1-\beta)\right) \\
& \times\left(\left\|x_{n}-x_{n+1}\right\|+\left\|x_{n+1}-B p_{k}\right\|\right) \\
& +\left(1-\alpha_{n}\right)(1-\beta)\left(\left\|x_{n}-x_{n+1}\right\|+\left\|x_{n+1}-p_{k}\right\|\right) \\
& +\left(1-\alpha_{n}\right)\left[(1-\beta)\left\|B_{n} p_{k}-B p_{k}\right\|\right. \\
& \left.+\delta_{n}\left\|S_{n} G x_{n}-B_{n} x_{n}\right\|\right] \\
& =\alpha_{n}\left\|f\left(x_{n}\right)-x_{n}\right\|+\left(\beta+\alpha_{n}(1-\beta)\right) \\
& \times\left\|x_{n+1}-B p_{k}\right\|+\left(1-\alpha_{n}\right)(1-\beta)\left\|x_{n+1}-p_{k}\right\| \\
& +\left(1-\alpha_{n}\right)\left[(1-\beta)\left\|B_{n} p_{k}-B p_{k}\right\|\right. \\
& \left.+\delta_{n}\left\|S_{n} G x_{n}-B_{n} x_{n}\right\|\right]+\left\|x_{n}-x_{n+1}\right\| .
\end{aligned}
$$

So, it immediately follows from $0 \leq \alpha_{n} \leq 1-\rho$, for all $n \geq n_{0}$, that

$$
\left\|x_{n+1}-B p_{k}\right\|
$$

$$
\begin{aligned}
\leq & \left\|x_{n+1}-p_{k}\right\|+\left\|B_{n} p_{k}-B p_{k}\right\|+\frac{1}{\left(1-\alpha_{n}\right)(1-\beta)} \\
& \times\left(\left\|\alpha_{n}\left(x_{n}-f\left(x_{n}\right)\right)\right\|+\left\|x_{n}-x_{n+1}\right\|\right) \\
& +\frac{\delta_{n}}{1-\beta}\left\|S_{n} G x_{n}-B_{n} x_{n}\right\| \\
\leq & \left\|x_{n+1}-p_{k}\right\|+\left\|B_{n} p_{k}-B p_{k}\right\| \\
& +\left\|S_{n} G x_{n}-B_{n} x_{n}\right\|+\frac{1}{\rho(1-\beta)} \\
& \times\left(\left\|\alpha_{n}\left(x_{n}-f\left(x_{n}\right)\right)\right\|+\left\|x_{n}-x_{n+1}\right\|\right) \\
= & \left\|x_{n+1}-p_{k}\right\|+\theta_{n}, \quad \forall n \geq n_{0},
\end{aligned}
$$


where $\theta_{n}=\left\|B_{n} p_{k}-B p_{k}\right\|+\left\|S_{n} G x_{n}-B_{n} x_{n}\right\|+(1 / \rho(1-$ $\beta))\left(\left\|\alpha_{n}\left(x_{n}-f\left(x_{n}\right)\right)\right\|+\left\|x_{n}-x_{n+1}\right\|\right)$. Since $\lim _{n \rightarrow \infty} \| B_{n} p_{k}-$ $B p_{k}\left\|=\lim _{n \rightarrow \infty}\right\| S_{n} G x_{n}-B_{n} x_{n}\left\|=\lim _{n \rightarrow \infty}\right\| \alpha_{n}\left(x_{n}-f\left(x_{n}\right)\right) \|=$ $\lim _{n \rightarrow \infty}\left\|x_{n}-x_{n+1}\right\|=0$, we know that $\theta_{n} \rightarrow 0$ as $n \rightarrow \infty$.

From (95), we obtain

$$
\begin{aligned}
\left\|x_{n+1}-B p_{k}\right\|^{2} \leq & \left\|x_{n+1}-p_{k}\right\|^{2} \\
& +\theta_{n}\left(2\left\|x_{n+1}-p_{k}\right\|+\theta_{n}\right), \quad \forall n \geq n_{0} .
\end{aligned}
$$

For any Banach limit $\mu$, from (96), we derive

$$
\begin{aligned}
\mu_{n}\left\|x_{n}-B p_{k}\right\|^{2} & =\mu_{n}\left\|x_{n+1}-B p_{k}\right\|^{2} \\
& \leq \mu_{n}\left\|x_{n+1}-p_{k}\right\|^{2}=\mu_{n}\left\|x_{n}-p_{k}\right\|^{2} .
\end{aligned}
$$

In addition, note that

$$
\begin{aligned}
&\left\|x_{n}-G p_{k}\right\|^{2} \\
& \leq\left\|x_{n}-G x_{n}+G x_{n}-G p_{k}\right\|^{2} \\
& \leq\left(\left\|x_{n}-G x_{n}\right\|+\left\|x_{n}-p_{k}\right\|\right)^{2} \\
&=\left\|x_{n}-p_{k}\right\|^{2}+\left\|x_{n}-G x_{n}\right\| \\
& \times\left(2\left\|x_{n}-p_{k}\right\|+\left\|x_{n}-G x_{n}\right\|\right), \\
& \| x_{n}- S p_{k} \|^{2} \\
& \leq\left\|x_{n}-S x_{n}+S x_{n}-S p_{k}\right\|^{2} \\
& \leq\left(\left\|x_{n}-S x_{n}\right\|+\left\|x_{n}-p_{k}\right\|\right)^{2} \\
&=\left\|x_{n}-p_{k}\right\|^{2}+\left\|x_{n}-S x_{n}\right\| \\
& \times\left(2\left\|x_{n}-p_{k}\right\|+\left\|x_{n}-S x_{n}\right\|\right) .
\end{aligned}
$$

It is easy to see from (81) and (91) that

$$
\begin{aligned}
& \mu_{n}\left\|x_{n}-G p_{k}\right\|^{2} \leq \mu_{n}\left\|x_{n}-p_{k}\right\|^{2}, \\
& \mu_{n}\left\|x_{n}-S p_{k}\right\|^{2} \leq \mu_{n}\left\|x_{n}-p_{k}\right\|^{2} .
\end{aligned}
$$

Utilizing (97) and (99), we deduce that

$$
\begin{aligned}
\mu_{n}\left\|x_{n}-V p_{k}\right\|^{2} & \\
= & \mu_{n} \|\left(1-\theta_{1}-\theta_{2}\right)\left(x_{n}-S p_{k}\right) \\
& \quad+\theta_{1}\left(x_{n}-B p_{k}\right)+\theta_{2}\left(x_{n}-G p_{k}\right) \|^{2} \\
\leq & \left(1-\theta_{1}-\theta_{2}\right) \mu_{n}\left\|x_{n}-S p_{k}\right\|^{2} \\
& +\theta_{1} \mu_{n}\left\|x_{n}-B p_{k}\right\|^{2}+\theta_{2} \mu_{n}\left\|x_{n}-G p_{k}\right\|^{2} \\
\leq & \mu_{n}\left\|x_{n}-p_{k}\right\|^{2} .
\end{aligned}
$$

Also, observe that

$$
x_{n}-p_{k}=\frac{1}{k}\left(x_{n}-f\left(p_{k}\right)\right)+\left(1-\frac{1}{k}\right)\left(x_{n}-V p_{k}\right) ;
$$

that is,

$$
\left(1-\frac{1}{k}\right)\left(x_{n}-V p_{k}\right)=x_{n}-p_{k}-\frac{1}{k}\left(x_{n}-f\left(p_{k}\right)\right) .
$$

It follows from Lemma 8 (ii) and (102) that

$$
\begin{aligned}
(1 & \left.-\frac{1}{k}\right)^{2}\left\|x_{n}-V p_{k}\right\|^{2} \\
& \geq\left\|x_{n}-p_{k}\right\|^{2}-\frac{2}{k}\left\langle x_{n}-p_{k}+p_{k}-f\left(p_{k}\right), J\left(x_{n}-p_{k}\right)\right\rangle \\
& =\left(1-\frac{2}{k}\right)\left\|x_{n}-p_{k}\right\|^{2}+\frac{2}{k}\left\langle f\left(p_{k}\right)-p_{k}, J\left(x_{n}-p_{k}\right)\right\rangle .
\end{aligned}
$$

So by (100) and (103), we have

$$
\begin{aligned}
\left(1-\frac{1}{k}\right)^{2} \mu_{n}\left\|x_{n}-p_{k}\right\|^{2} & \\
\geq & \left(1-\frac{2}{k}\right) \mu_{n}\left\|x_{n}-p_{k}\right\|^{2} \\
& +\frac{2}{k} \mu_{n}\left\langle f\left(p_{k}\right)-p_{k}, J\left(x_{n}-p_{k}\right)\right\rangle,
\end{aligned}
$$

and hence

$$
\frac{1}{k^{2}} \mu_{n}\left\|x_{n}-p_{k}\right\|^{2} \geq \frac{2}{k} \mu_{n}\left\langle f\left(p_{k}\right)-p_{k}, J\left(x_{n}-p_{k}\right)\right\rangle .
$$

This implies that

$$
\frac{1}{2 k} \mu_{n}\left\|x_{n}-p_{k}\right\|^{2} \geq \mu_{n}\left\langle f\left(p_{k}\right)-p_{k}, J\left(x_{n}-p_{k}\right)\right\rangle .
$$

Since $p_{k} \rightarrow q \in \operatorname{Fix}(V)=F$ as $k \rightarrow \infty$, by the uniform Frechet differentiability of the norm of $X$ we have

$$
\mu_{n}\left\langle f(q)-q, J\left(x_{n}-q\right)\right\rangle \leq 0 .
$$

On the other hand, from (49) and the norm-to-norm uniform continuity of $J$ on bounded subsets of $X$, it follows that

$$
\lim _{n \rightarrow \infty}\left|\left\langle f(q)-q, J\left(x_{n+1}-q\right)\right\rangle-\left\langle f(q)-q, J\left(x_{n}-q\right)\right\rangle\right|=0 .
$$

So, utilizing Lemma 18 we deduce from (107) and (108) that

$$
\limsup _{n \rightarrow \infty}\left\langle f(q)-q, J\left(x_{n}-q\right)\right\rangle \leq 0,
$$

which together with (49) and the norm-to-norm uniform continuity of $J$ on bounded subsets of $X$, implies that

$$
\limsup _{n \rightarrow \infty}\left\langle f(q)-q, J\left(x_{n+1}-q\right)\right\rangle \leq 0 .
$$


Finally, let us show that $x_{n} \rightarrow q$ as $n \rightarrow \infty$. Utilizing Lemma 8 (i), from (42) and the convexity of $\|\cdot\|^{2}$, we get

$$
\begin{aligned}
\| y_{n}- & q \|^{2} \\
\leq & \beta_{n}\left\|x_{n}-q\right\|^{2}+\gamma_{n}\left\|B_{n} x_{n}-q\right\|^{2} \\
& +\delta_{n}\left\|S_{n} G x_{n}-q\right\|^{2} \\
\leq & \beta_{n}\left\|x_{n}-q\right\|^{2}+\gamma_{n}\left\|x_{n}-q\right\|^{2} \\
& +\delta_{n}\left\|x_{n}-q\right\|^{2} \\
= & \left\|x_{n}-q\right\|^{2}, \\
\| x_{n+1} & -q \|^{2} \\
= & \| \alpha_{n}\left(f\left(x_{n}\right)-f(q)\right)+\left(1-\alpha_{n}\right)\left(y_{n}-q\right) \\
& +\alpha_{n}(f(q)-q) \|^{2} \\
\leq & \left\|\alpha_{n}\left(f\left(x_{n}\right)-f(q)\right)+\left(1-\alpha_{n}\right)\left(y_{n}-q\right)\right\|^{2} \\
& +2 \alpha_{n}\left\langle f(q)-q, J\left(x_{n+1}-q\right)\right\rangle \\
\leq & \alpha_{n}\left\|f\left(x_{n}\right)-f(q)\right\|^{2}+\left(1-\alpha_{n}\right)\left\|y_{n}-q\right\|^{2} \\
& +2 \alpha_{n}\left\langle f(q)-q, J\left(x_{n+1}-q\right)\right\rangle \\
\leq & \alpha_{n} \rho\left\|x_{n}-q\right\|^{2}+\left(1-\alpha_{n}\right)\left\|x_{n}-q\right\|^{2} \\
& +2 \alpha_{n}\left\langle f(q)-q, J\left(x_{n+1}-q\right)\right\rangle \\
= & \left(1-\alpha_{n}(1-\rho)\right)\left\|x_{n}-q\right\|^{2} \\
& +2 \alpha_{n}\left\langle f(q)-q, J\left(x_{n+1}-q\right)\right\rangle \\
= & \left(1-\alpha_{n}(1-\rho)\right)\left\|x_{n}-q\right\|^{2} \\
& +\alpha_{n}(1-\rho) \frac{2\left\langle f(q)-q, J\left(x_{n+1}-q\right)\right\rangle}{1-\rho} \\
&
\end{aligned}
$$

Applying Lemma 7 to (112), we obtain that $x_{n} \rightarrow q$ as $n \rightarrow$ $\infty$.

Conversely, if $x_{n} \rightarrow q \in F$ as $n \rightarrow \infty$, then from (42) it follows that

$$
\begin{aligned}
\left\|y_{n}-q\right\| \\
\leq \beta_{n}\left\|x_{n}-q\right\|+\gamma_{n}\left\|B_{n} x_{n}-q\right\| \\
\quad+\delta_{n}\left\|S_{n} G x_{n}-q\right\| \\
\leq \beta_{n}\left\|x_{n}-q\right\|+\gamma_{n}\left\|x_{n}-q\right\|+\delta_{n}\left\|x_{n}-q\right\| \\
=\left\|x_{n}-q\right\| \longrightarrow 0 \quad \text { as } n \longrightarrow \infty,
\end{aligned}
$$

that is, $y_{n} \rightarrow q$. Again from (42) we obtain that

$$
\begin{aligned}
\| \alpha_{n}( & \left.f\left(x_{n}\right)-x_{n}\right) \| \\
& =\left\|x_{n+1}-x_{n}-\left(1-\alpha_{n}\right)\left(y_{n}-x_{n}\right)\right\| \\
& \leq\left\|x_{n+1}-x_{n}\right\|+\left(1-\alpha_{n}\right)\left\|y_{n}-x_{n}\right\|
\end{aligned}
$$

$$
\begin{aligned}
\leq & \left\|x_{n+1}-q\right\|+\left\|x_{n}-q\right\| \\
& +\left(1-\alpha_{n}\right)\left(\left\|y_{n}-q\right\|+\left\|x_{n}-q\right\|\right) \\
\leq & \left\|x_{n+1}-q\right\|+2\left\|x_{n}-q\right\|+\left\|y_{n}-q\right\| .
\end{aligned}
$$

Since $x_{n} \rightarrow q$ and $y_{n} \rightarrow q$, we get $\alpha_{n}\left(f\left(x_{n}\right)-x_{n}\right) \rightarrow 0$. This completes the proof.

Corollary 25. Let $C$ be a nonempty closed convex subset of a uniformly convex and 2-uniformly smooth Banach space X. Let $\Pi_{C}$ be a sunny nonexpansive retraction from $X$ onto $C$. Let $\left\{\rho_{n}\right\}_{n=0}^{\infty}$ be a sequence of positive numbers in $(0, b]$ for some $b \in(0,1)$ and $A_{i}: C \rightarrow E$ an $\widehat{\alpha}_{i}$-inverse strongly accretive mapping for each $i=0,1, \ldots$ Define a mapping $G_{i}: C \rightarrow C$ by $\Pi_{C}\left(I-\lambda_{i} A_{i}\right) x=G_{i} x$ for all $x \in C$ and $i=0,1, \ldots$, where $\lambda_{i} \in\left(0, \widehat{\alpha}_{i} / \kappa^{2}\right]$ and $\kappa$ is the 2-uniformly smooth constant of $X$. Let $B_{n}: C \rightarrow C$ be the $W$-mapping generated by $G_{n}, G_{n-1}, \ldots, G_{0}$ and $\rho_{n}, \rho_{n-1}, \ldots, \rho_{0}$. Let $V: C \rightarrow C$ be an $\alpha$-strictly pseudocontractive mapping. Let $f: C \rightarrow C$ be a contraction with coefficient $\rho \in(0,1)$. Let $\left\{S_{i}\right\}_{i=0}^{\infty}$ be a countable family of nonexpansive mappings of $C$ into itself such that $F=\left(\bigcap_{i=0}^{\infty} \operatorname{Fix}\left(S_{i}\right)\right) \cap \operatorname{Fix}(V) \cap\left(\bigcap_{i=0}^{\infty} \operatorname{VI}\left(C, A_{i}\right)\right) \neq \emptyset$. For arbitrarily given $x_{0} \in C$, let $\left\{x_{n}\right\}$ be the sequence generated by

$$
\begin{gathered}
y_{n}=\beta_{n} x_{n}+\gamma_{n} B_{n} x_{n}+\delta_{n} S_{n}((1-l) I+l V) x_{n}, \\
x_{n+1}=\alpha_{n} f\left(x_{n}\right)+\left(1-\alpha_{n}\right) y_{n}, \quad \forall n \geq 0,
\end{gathered}
$$

where $0<l<\alpha / \kappa^{2},\left\{\alpha_{n}\right\},\left\{\beta_{n}\right\},\left\{\gamma_{n}\right\}$, and $\left\{\delta_{n}\right\}$ are the sequences in $[0,1]$ such that $\beta_{n}+\gamma_{n}+\delta_{n}=1$ for all $n \geq 0$. Suppose that the following conditions hold:

(i) $\sum_{n=0}^{\infty} \alpha_{n}=\infty$ and $0 \leq \alpha_{n} \leq 1-\rho$, for all $n \geq n_{0}$ for some integer $n_{0} \geq 0$;

(ii) $\liminf _{n \rightarrow \infty} \gamma_{n}>0$ and $\liminf _{n \rightarrow \infty} \delta_{n}>0$;

(iii) $\lim _{n \rightarrow \infty}\left(\mid \alpha_{n+1} /\left(1-\left(1-\alpha_{n+1}\right) \beta_{n+1}\right)-\alpha_{n} /(1-(1-\right.$ $\left.\left.\left.\alpha_{n}\right) \beta_{n}\right)|+| \delta_{n+1} /\left(1-\beta_{n+1}\right)-\delta_{n} /\left(1-\beta_{n}\right) \mid\right)=0$;

(iv) $0<\liminf _{n \rightarrow \infty} \beta_{n} \leq \lim \sup _{n \rightarrow \infty} \beta_{n}<1$.

Assume that $\sum_{n=0}^{\infty} \sup _{x \in D}\left\|S_{n+1} x-S_{n} x\right\|<\infty$ for any bounded subset $D$ of $C$ and let $S$ be a mapping of $C$ into itself defined by $S x=\lim _{n \rightarrow \infty} S_{n} x$ for all $x \in C$ and suppose that Fix $(S)=$ $\bigcap_{i=0}^{\infty} \operatorname{Fix}\left(S_{i}\right)$. Then, there hold the following:

(I) $\lim _{n \rightarrow \infty}\left\|x_{n+1}-x_{n}\right\|=0$;

(II) $x_{n} \rightarrow q \Leftrightarrow \alpha_{n}\left(f\left(x_{n}\right)-x_{n}\right) \rightarrow 0$ provided $\beta_{n} \equiv \beta$ for some fixed $\beta \in(0,1)$, where $q \in F$ solves the following VIP

$$
\langle q-f(q), J(q-p)\rangle \leq 0, \quad \forall p \in F .
$$

Proof. In Theorem 24, we put $B_{1}=I-V, B_{2}=0$, and $\mu_{1}=l$, where $0<l<\alpha / \kappa^{2}$. Then, GSVI (13) is equivalent to the VIP of finding $x^{*} \in C$ such that

$$
\left\langle B_{1} x^{*}, J\left(x-x^{*}\right)\right\rangle \geq 0, \quad \forall x \in C .
$$


In this case, $B_{1}: C \rightarrow X$ is $\alpha$-inverse strongly accretive. It is not hard to see that $\operatorname{Fix}(V)=\operatorname{VI}\left(C, B_{1}\right)$. As a matter of fact, we have, for $l>0$,

$$
\begin{aligned}
u \in \mathrm{VI} & \left(C, B_{1}\right) \\
& \Longleftrightarrow\left\langle B_{1} u, J(y-u)\right\rangle \geq 0 \quad \forall y \in C \\
& \Longleftrightarrow\left\langle u-l B_{1} u-u, J(u-y)\right\rangle \geq 0 \quad \forall y \in C \\
& \Longleftrightarrow u=\Pi_{C}\left(u-l B_{1} u\right) \\
& \Longleftrightarrow u=\Pi_{C}(u-l u+l V u) \\
& \Longleftrightarrow\langle u-l u+l V u-u, J(u-y)\rangle \geq 0 \quad \forall y \in C \\
& \Longleftrightarrow\langle u-V u, J(u-y)\rangle \leq 0 \quad \forall y \in C \\
& \Longleftrightarrow u=V u \\
& \Longleftrightarrow u \in \operatorname{Fix}(V) .
\end{aligned}
$$

Accordingly, we know that $F=\left(\bigcap_{i=0}^{\infty} \operatorname{Fix}\left(S_{i}\right)\right) \cap \Omega \cap$ $\left(\bigcap_{i=0}^{\infty} \operatorname{VI}\left(C, A_{i}\right)\right)=\left(\bigcap_{i=0}^{\infty} \operatorname{Fix}\left(S_{i}\right)\right) \cap \operatorname{Fix}(V) \cap\left(\bigcap_{i=0}^{\infty} \operatorname{VI}\left(C, A_{i}\right)\right)$, and

$$
\begin{aligned}
\Pi_{C}\left(I-\mu_{1} B_{1}\right) \Pi_{C}\left(I-\mu_{2} B_{2}\right) x_{n} \\
=\Pi_{C}\left(I-\mu_{1} B_{1}\right) x_{n} \\
=\Pi_{C}\left((1-l) x_{n}+l V x_{n}\right) \\
=((1-l) I+l V) x_{n} .
\end{aligned}
$$

So, the scheme (42) reduces to (115). Therefore, the desired result follows from Theorem 24 .

Here, we prove the following important lemmas which will be used in the sequel.

Lemma 26. Let $C$ be a nonempty closed convex subset of a smooth Banach space $X$ and let the mapping $B_{i}: C \rightarrow X$ be $\lambda_{i}$-strictly pseudocontractive and $\alpha_{i}$-strongly accretive with $\alpha_{i}+\lambda_{i} \geq 1$ for $i=1,2$. Then, for $\mu_{i} \in(0,1]$ one has

$$
\begin{aligned}
& \left\|\left(I-\mu_{i} B_{i}\right) x-\left(I-\mu_{i} B_{i}\right) y\right\| \\
& \quad \leq\left\{\sqrt{\frac{1-\alpha_{i}}{\lambda_{i}}}+\left(1-\mu_{i}\right)\left(1+\frac{1}{\lambda_{i}}\right)\right\}\|x-y\|,
\end{aligned}
$$

$$
\forall x, y \in C,
$$

for $i=1$, 2. In particular, if $1-\left(\lambda_{i} /\left(1+\lambda_{i}\right)\right)\left(1-\sqrt{\left(1-\alpha_{i}\right) / \lambda_{i}}\right) \leq$ $\mu_{i} \leq 1$, then $I-\mu_{i} B_{i}$ is nonexpansive for $i=1,2$.

Proof. Taking into account the $\lambda_{i}$-strict pseudocontractivity of $B_{i}$, we derive for every $x, y \in C$

$$
\begin{aligned}
\lambda_{i}\left\|\left(I-B_{i}\right) x-\left(I-B_{i}\right) y\right\|^{2} \\
\quad \leq\left\langle\left(I-B_{i}\right) x-\left(I-B_{i}\right) y, J(x-y)\right\rangle \\
\leq\left\|\left(I-B_{i}\right) x-\left(I-B_{i}\right) y\right\|\|x-y\|,
\end{aligned}
$$

which implies that

$$
\left\|\left(I-B_{i}\right) x-\left(I-B_{i}\right) y\right\| \leq \frac{1}{\lambda_{i}}\|x-y\| .
$$

Hence,

$$
\begin{aligned}
\left\|B_{i} x-B_{i} y\right\| & \leq\left\|\left(I-B_{i}\right) x-\left(I-B_{i}\right) y\right\|+\|x-y\| \\
& \leq\left(1+\frac{1}{\lambda_{i}}\right)\|x-y\| .
\end{aligned}
$$

Utilizing the $\alpha_{i}$-strong accretivity and $\lambda_{i}$-strict pseudocontractivity of $B_{i}$, we get

$$
\begin{aligned}
\lambda_{i} \|( & \left.-B_{i}\right) x-\left(I-B_{i}\right) y \|^{2} \\
& \leq\|x-y\|^{2}-\left\langle B_{i} x-B_{i} y, J(x-y)\right\rangle \\
& \leq\left(1-\alpha_{i}\right)\|x-y\|^{2} .
\end{aligned}
$$

So, we have

$$
\left\|\left(I-B_{i}\right) x-\left(I-B_{i}\right) y\right\| \leq \sqrt{\frac{1-\alpha_{i}}{\lambda_{i}}}\|x-y\| .
$$

Therefore, for $\mu_{i} \in(0,1]$ we have

$$
\begin{aligned}
\|(I & \left.-\mu_{i} B_{i}\right) x-\left(I-\mu_{i} B_{i}\right) y \| \\
& \leq\left\|\left(I-B_{i}\right) x-\left(I-B_{i}\right) y\right\|+\left(1-\mu_{i}\right)\left\|B_{i} x-B_{i} y\right\| \\
& \leq \sqrt{\frac{1-\alpha_{i}}{\lambda_{i}}}\|x-y\|+\left(1-\mu_{i}\right)\left(1+\frac{1}{\lambda_{i}}\right)\|x-y\| \\
& =\left\{\sqrt{\frac{1-\alpha_{i}}{\lambda_{i}}}+\left(1-\mu_{i}\right)\left(1+\frac{1}{\lambda_{i}}\right)\right\}\|x-y\| .
\end{aligned}
$$

Since $1-\left(\lambda_{i} /\left(1+\lambda_{i}\right)\right)\left(1-\sqrt{\left(1-\alpha_{i}\right) / \lambda_{i}}\right) \leq \mu_{i} \leq 1$, it follows immediately that

$$
\sqrt{\frac{1-\alpha_{i}}{\lambda_{i}}}+\left(1-\mu_{i}\right)\left(1+\frac{1}{\lambda_{i}}\right) \leq 1 .
$$

This implies that $I-\mu_{i} B_{i}$ is nonexpansive for $i=1,2$.

Lemma 27. Let $C$ be a nonempty closed convex subset of a smooth Banach space $X$. Let $\Pi_{C}$ be a sunny nonexpansive retraction from $X$ onto $C$ and let the mapping $B_{i}: C \rightarrow X$ be $\lambda_{i}$-strictly pseudocontractive and $\alpha_{i}$-strongly accretive with $\alpha_{i}+\lambda_{i} \geq 1$ for $i=1,2$. Let $G: C \rightarrow C$ be the mapping defined by

$$
G(x)=\Pi_{C}\left[\Pi_{C}\left(x-\mu_{2} B_{2} x\right)-\mu_{1} B_{1} \Pi_{C}\left(x-\mu_{2} B_{2} x\right)\right],
$$

$\forall x \in C$.

If $1-\left(\lambda_{i} /\left(1+\lambda_{i}\right)\right)\left(1-\sqrt{\left(1-\alpha_{i}\right) / \lambda_{i}}\right) \leq \mu_{i} \leq 1$, then $G: C \rightarrow C$ is nonexpansive. 
Proof. According to Lemma 26, we know that $I-\mu_{i} B_{i}$ is nonexpansive for $i=1,2$. Hence, for all $x, y \in C$, we have

$$
\begin{aligned}
&\|G(x)-G(y)\| \\
&=\| \Pi_{C}\left[\Pi_{C}\left(x-\mu_{2} B_{2} x\right)-\mu_{1} B_{1} \Pi_{C}\left(x-\mu_{2} B_{2} x\right)\right] \\
& \quad \quad-\Pi_{C}\left[\Pi_{C}\left(y-\mu_{2} B_{2} y\right)-\mu_{1} B_{1} \Pi_{C}\left(y-\mu_{2} B_{2} y\right)\right] \| \\
&=\| \Pi_{C}\left(I-\mu_{1} B_{1}\right) \Pi_{C}\left(I-\mu_{2} B_{2}\right) x \\
& \quad-\Pi_{C}\left(I-\mu_{1} B_{1}\right) \Pi_{C}\left(I-\mu_{2} B_{2}\right) y \| \\
& \leq \|\left(I-\mu_{1} B_{1}\right) \Pi_{C}\left(I-\mu_{2} B_{2}\right) x \\
& \quad-\left(I-\mu_{1} B_{1}\right) \Pi_{C}\left(I-\mu_{2} B_{2}\right) y \| \\
& \leq\left\|\Pi_{C}\left(I-\mu_{2} B_{2}\right) x-\Pi_{C}\left(I-\mu_{2} B_{2}\right) y\right\| \\
& \leq\left\|\left(I-\mu_{2} B_{2}\right) x-\left(I-\mu_{2} B_{2}\right) y\right\| \\
& \leq\|x-y\| .
\end{aligned}
$$

This shows that $G: C \rightarrow C$ is nonexpansive. This completes the proof.

Theorem 28. Let $C$ be a nonempty closed convex subset of a uniformly convex Banach space $X$ which has a uniformly Gateaux differentiable norm. Let $\Pi_{C}$ be a sunny nonexpansive retraction from $X$ onto $C$. Let $\left\{\rho_{n}\right\}_{n=0}^{\infty}$ be a sequence of positive numbers in $(0, b]$ for some $b \in(0,1)$ and $A_{i}: C \rightarrow X$ be $\xi_{i}$-strictly pseudocontractive and $\widehat{\alpha}_{i}$-strongly accretive with $\xi_{i}+\widehat{\alpha}_{i} \geq 1$ for each $i=0,1, \ldots$. Define a mapping $G_{i}: C \rightarrow C$ by $\Pi_{C}\left(I-\lambda_{i} A_{i}\right) x=G_{i} x$ for all $x \in C$ and $i=0,1, \ldots$, where $1-\left(\xi_{i} /\left(1+\xi_{i}\right)\right)\left(1-\sqrt{\left(1-\widehat{\alpha}_{i}\right) / \xi_{i}}\right) \leq \lambda_{i} \leq 1$ for all $i=0,1, \ldots$.. Let $B_{n}: C \rightarrow C$ be the $W$-mapping generated by $G_{n}, G_{n-1}, \ldots, G_{0}$ and $\rho_{n}, \rho_{n-1}, \ldots, \rho_{0}$. Let the mapping $B_{i}$ : $C \rightarrow X \zeta_{i}$-strictly pseudocontractive and $\widehat{\beta}_{i}$-strongly accretive with $\zeta_{i}+\widehat{\beta}_{i} \geq 1$ for $i=1,2$. Let $f: C \rightarrow C$ be a contraction with coefficient $\rho \in(0,1)$. Let $\left\{S_{i}\right\}_{i=0}^{\infty}$ be a countable family of nonexpansive mappings of $C$ into itself such that $F=$ $\left(\bigcap_{i=0}^{\infty} \operatorname{Fix}\left(S_{i}\right)\right) \cap \Omega \cap\left(\bigcap_{i=0}^{\infty} \operatorname{VI}\left(C, A_{i}\right)\right) \neq \emptyset$, where $\Omega$ is the fixed point set of the mapping $G=\Pi_{C}\left(I-\mu_{1} B_{1}\right) \Pi_{C}\left(I-\mu_{2} B_{2}\right)$ with $1-\left(\zeta_{i} /\left(1+\zeta_{i}\right)\right)\left(1-\sqrt{\left(1-\widehat{\beta}_{i}\right) / \zeta_{i}}\right) \leq \mu_{i} \leq 1$ for $i=1,2$. For arbitrarily given $x_{0} \in C$, let $\left\{x_{n}\right\}$ be the sequence generated by

$$
\begin{gathered}
y_{n}=\beta_{n} x_{n}+\gamma_{n} B_{n} x_{n}+\delta_{n} S_{n} G x_{n}, \\
x_{n+1}=\alpha_{n} f\left(x_{n}\right)+\sigma_{n} G x_{n}+\left(1-\alpha_{n}-\sigma_{n}\right) y_{n}, \quad \forall n \geq 0,
\end{gathered}
$$

where $\left\{\sigma_{n}\right\},\left\{\alpha_{n}\right\},\left\{\beta_{n}\right\},\left\{\gamma_{n}\right\}$, and $\left\{\delta_{n}\right\}$ are the sequences in $[0,1]$ such that $\beta_{n}+\gamma_{n}+\delta_{n}=1$ and $\alpha_{n}+\sigma_{n} \leq 1$ for all $n \geq 0$. Suppose that the following conditions hold:

(i) $\sum_{n=0}^{\infty} \alpha_{n}=\infty$ and $0 \leq \alpha_{n}+\sigma_{n} \leq 1-\rho$, for all $n \geq n_{0}$ for some integer $n_{0} \geq 0$;

(ii) $\liminf _{n \rightarrow \infty} \sigma_{n}>0$, $\liminf _{n \rightarrow \infty} \gamma_{n}>0$ and $\liminf _{n \rightarrow \infty} \delta_{n}>0$; (iii) $\lim _{n \rightarrow \infty}\left(\mid \alpha_{n+1} /\left(1-\left(1-\alpha_{n+1}-\sigma_{n+1}\right) \beta_{n+1}\right)-\left(\alpha_{n} /(1-(1-\right.\right.$ $\left.\left.\left.\alpha_{n}-\sigma_{n}\right) \beta_{n}\right)\right)|+| \sigma_{n+1} /\left(1-\left(1-\alpha_{n+1}-\sigma_{n+1}\right) \beta_{n+1}\right)-\left(\sigma_{n} /(1-\right.$ $\left.\left.\left.\left(1-\alpha_{n}-\sigma_{n}\right) \beta_{n}\right)\right)|+| \delta_{n+1} /\left(1-\beta_{n+1}\right)-\delta_{n} /\left(1-\beta_{n}\right) \mid\right)=0 ;$

(iv) $0<\liminf _{n \rightarrow \infty} \beta_{n} \leq \lim \sup _{n \rightarrow \infty} \beta_{n}<1$.

Assume that $\sum_{n=0}^{\infty} \sup _{x \in D}\left\|S_{n+1} x-S_{n} x\right\|<\infty$ for any bounded subset $D$ of $C$ and let $S$ be a mapping of $C$ into itself defined by $S x=\lim _{n \rightarrow \infty} S_{n} x$ for all $x \in C$ and suppose that $\operatorname{Fix}(S)=$ $\bigcap_{i=0}^{\infty} \operatorname{Fix}\left(S_{i}\right)$. Then there hold the following:

(I) $\lim _{n \rightarrow \infty}\left\|x_{n+1}-x_{n}\right\|=0$;

(II) $x_{n} \rightarrow q \Leftrightarrow \alpha_{n}\left(f\left(x_{n}\right)-x_{n}\right) \rightarrow 0$ provided $\beta_{n} \equiv \beta$ for some fixed $\beta \in(0,1)$, where $q \in F$ solves the following VIP

$$
\langle q-f(q), J(q-p)\rangle \leq 0, \quad \forall p \in F .
$$

Proof. First of all, take a fixed $p \in F$ arbitrarily. Then we obtain $p=G p, p=B_{n} p$ and $S_{n} p=p$ for all $n \geq 0$. By Lemma 27, we get from (130)

$$
\begin{aligned}
& \left\|y_{n}-p\right\| \\
& \quad \leq \beta_{n}\left\|x_{n}-p\right\|+\gamma_{n}\left\|B_{n} x_{n}-p\right\|+\delta_{n}\left\|S_{n} G x_{n}-p\right\| \\
& \quad \leq \beta_{n}\left\|x_{n}-p\right\|+\gamma_{n}\left\|x_{n}-p\right\|+\delta_{n}\left\|x_{n}-p\right\| \\
& \quad=\left\|x_{n}-p\right\|,
\end{aligned}
$$

and hence

$$
\begin{aligned}
\left\|x_{n+1}-p\right\| & \\
\leq & \alpha_{n}\left\|f\left(x_{n}\right)-p\right\|+\sigma_{n}\left\|G x_{n}-p\right\| \\
& +\left(1-\alpha_{n}-\sigma_{n}\right)\left\|y_{n}-p\right\| \\
\leq & \alpha_{n}\left(\left\|f\left(x_{n}\right)-f(p)\right\|+\|f(p)-p\|\right) \\
& +\sigma_{n}\left\|x_{n}-p\right\|+\left(1-\alpha_{n}-\sigma_{n}\right)\left\|x_{n}-p\right\| \\
\leq & \alpha_{n}\left(\rho\left\|x_{n}-p\right\|+\|f(p)-p\|\right) \\
& +\sigma_{n}\left\|x_{n}-p\right\|+\left(1-\alpha_{n}-\sigma_{n}\right)\left\|x_{n}-p\right\| \\
= & \left(1-\alpha_{n}(1-\rho)\right)\left\|x_{n}-p\right\|+\alpha_{n}\|f(p)-p\| \\
= & \left(1-\alpha_{n}(1-\rho)\right)\left\|x_{n}-p\right\|+\alpha_{n}(1-\rho) \frac{\|f(p)-p\|}{1-\rho} \\
\leq & \max \left\{\left\|x_{n}-p\right\|, \frac{\|f(p)-p\|}{1-\rho}\right\} .
\end{aligned}
$$

By induction, we have

$$
\left\|x_{n}-p\right\| \leq \max \left\{\left\|x_{0}-p\right\|, \frac{\|f(p)-p\|}{1-\rho}\right\}, \quad \forall n \geq 0,
$$

which implies that $\left\{x_{n}\right\}$ is bounded and so are the sequences $\left\{y_{n}\right\},\left\{G x_{n}\right\}$, and $\left\{f\left(x_{n}\right)\right\}$. 
Let us show that

$$
\lim _{n \rightarrow \infty}\left\|x_{n+1}-x_{n}\right\|=0 .
$$

As a matter of fact, put $\theta_{n}=\left(1-\alpha_{n}-\sigma_{n}\right) \beta_{n}$, for all $n \geq 0$. Then, it follows from (i) and (iv) that

$$
\begin{array}{r}
\beta_{n} \geq \theta_{n}=\left(1-\alpha_{n}-\sigma_{n}\right) \beta_{n} \geq(1-(1-\rho)) \beta_{n}=\rho \beta_{n}, \\
\forall n \geq n_{0},
\end{array}
$$

and hence

$$
0<\liminf _{n \rightarrow \infty} \theta_{n} \leq \limsup _{n \rightarrow \infty} \theta_{n}<1 .
$$

Define

$$
x_{n+1}=\theta_{n} x_{n}+\left(1-\theta_{n}\right) z_{n} .
$$

Observe that

$$
\begin{aligned}
& z_{n+1}-z_{n} \\
& =\frac{x_{n+2}-\theta_{n+1} x_{n+1}}{1-\theta_{n+1}}-\frac{x_{n+1}-\theta_{n} x_{n}}{1-\theta_{n}} \\
& =\left(\alpha_{n+1} f\left(x_{n+1}\right)+\sigma_{n+1} G x_{n+1}\right. \\
& \left.+\left(1-\alpha_{n+1}-\sigma_{n+1}\right) y_{n+1}-\theta_{n+1} x_{n+1}\right) \\
& \times\left(1-\theta_{n+1}\right)^{-1} \\
& -\frac{\alpha_{n} f\left(x_{n}\right)+\sigma_{n} G x_{n}+\left(1-\alpha_{n}-\sigma_{n}\right) y_{n}-\theta_{n} x_{n}}{1-\theta_{n}} \\
& =\left(\frac{\alpha_{n+1} f\left(x_{n+1}+\sigma_{n+1} G x_{n+1}\right)}{1-\theta_{n+1}}-\frac{\alpha_{n} f\left(x_{n}\right)+\sigma_{n} G x_{n}}{1-\theta_{n}}\right) \\
& -\frac{\left(1-\alpha_{n}-\sigma_{n}\right)\left[\beta_{n} x_{n}+\gamma_{n} B_{n} x_{n}+\delta_{n} S_{n} G x_{n}\right]-\theta_{n} x_{n}}{1-\theta_{n}} \\
& +\left(1-\alpha_{n+1}-\sigma_{n+1}\right) \\
& \times\left[\beta_{n+1} x_{n+1}+\gamma_{n+1} B_{n+1} x_{n+1}+\delta_{n+1} S_{n+1} G x_{n+1}\right] \\
& -\theta_{n+1} x_{n+1} \times\left(1-\theta_{n+1}\right)^{-1} \\
& =\left(\frac{\alpha_{n+1} f\left(x_{n+1}\right)+\sigma_{n+1} G x_{n+1}}{1-\theta_{n+1}}-\frac{\alpha_{n} f\left(x_{n}\right)+\sigma_{n} G x_{n}}{1-\theta_{n}}\right) \\
& +\frac{1-\alpha_{n+1}-\sigma_{n+1}}{1-\theta_{n+1}}\left(\gamma_{n+1} B_{n+1} x_{n+1}+\delta_{n+1} S_{n+1} G x_{n+1}\right) \\
& -\frac{1-\alpha_{n}-\sigma_{n}}{1-\theta_{n}}\left(\gamma_{n} B_{n} x_{n}+\delta_{n} S_{n} G x_{n}\right) \\
& =\left(\frac{\alpha_{n+1} f\left(x_{n+1}\right)+\sigma_{n+1} G x_{n+1}}{1-\theta_{n+1}}-\frac{\alpha_{n} f\left(x_{n}\right)+\sigma_{n} G x_{n}}{1-\theta_{n}}\right) \\
& +\frac{\left(1-\alpha_{n+1}-\sigma_{n+1}\right)\left(1-\beta_{n+1}\right)}{1-\theta_{n+1}}
\end{aligned}
$$

$$
\begin{gathered}
\times\left[\frac{\gamma_{n+1} B_{n+1} x_{n+1}+\delta_{n+1} S_{n+1} G x_{n+1}}{1-\beta_{n+1}}\right. \\
\left.-\frac{\gamma_{n} B_{n} x_{n}+\delta_{n} S_{n} G x_{n}}{1-\beta_{n}}\right]
\end{gathered}
$$$$
+\left[\frac{\left(1-\alpha_{n+1}-\sigma_{n+1}\right)\left(1-\beta_{n+1}\right)}{1-\theta_{n+1}}\right.
$$$$
\left.-\frac{\left(1-\alpha_{n}-\sigma_{n}\right)\left(1-\beta_{n}\right)}{1-\theta_{n}}\right]
$$$$
\times \frac{\gamma_{n} B_{n} x_{n}+\delta_{n} S_{n} G x_{n}}{1-\beta_{n}}
$$$$
=\frac{\alpha_{n+1}}{1-\theta_{n+1}}\left(f\left(x_{n+1}\right)-f\left(x_{n}\right)\right)
$$$$
+\frac{\sigma_{n+1}}{1-\theta_{n+1}}\left(G x_{n+1}-G x_{n}\right)
$$$$
+\left(\frac{\alpha_{n+1}}{1-\theta_{n+1}}-\frac{\alpha_{n}}{1-\theta_{n}}\right) f\left(x_{n}\right)
$$$$
+\left(\frac{\sigma_{n+1}}{1-\theta_{n+1}}-\frac{\sigma_{n}}{1-\theta_{n}}\right) G x_{n}
$$$$
+\frac{\left(1-\alpha_{n+1}-\sigma_{n+1}\right)\left(1-\beta_{n+1}\right)}{1-\theta_{n+1}}
$$$$
\times\left[\frac{\gamma_{n+1}}{\gamma_{n+1}+\delta_{n+1}}\left(B_{n+1} x_{n+1}-B_{n} x_{n}\right)\right.
$$$$
+\left(\frac{\gamma_{n+1}}{\gamma_{n+1}+\delta_{n+1}}-\frac{\gamma_{n}}{\gamma_{n}+\delta_{n}}\right) B_{n} x_{n}
$$$$
+\frac{\delta_{n+1}}{\gamma_{n+1}+\delta_{n+1}}\left(S_{n+1} G x_{n+1}-S_{n} G x_{n}\right)
$$$$
\left.+\left(\frac{\delta_{n+1}}{\gamma_{n+1}+\delta_{n+1}}-\frac{\delta_{n}}{\gamma_{n}+\delta_{n}}\right) S_{n} G x_{n}\right]
$$$$
-\left(\frac{\alpha_{n+1}+\sigma_{n+1}}{1-\theta_{n+1}}-\frac{\alpha_{n}+\sigma_{n}}{1-\theta_{n}}\right) \frac{\gamma_{n} B_{n} x_{n}+\delta_{n} S_{n} G x_{n}}{\gamma_{n}+\delta_{n}}
$$$$
=\frac{\alpha_{n+1}}{1-\theta_{n+1}}\left(f\left(x_{n+1}\right)-f\left(x_{n}\right)\right)
$$$$
+\frac{\sigma_{n+1}}{1-\theta_{n+1}}\left(G x_{n+1}-G x_{n}\right)
$$$$
+\left(\frac{\alpha_{n+1}}{1-\theta_{n+1}}-\frac{\alpha_{n}}{1-\theta_{n}}\right)\left(f\left(x_{n}\right)-\frac{\gamma_{n} B_{n} x_{n}+\delta_{n} S_{n} G x_{n}}{\gamma_{n}+\delta_{n}}\right)
$$$$
+\left(\frac{\sigma_{n+1}}{1-\theta_{n+1}}-\frac{\sigma_{n}}{1-\theta_{n}}\right)\left(G x_{n}-\frac{\gamma_{n} B_{n} x_{n}+\delta_{n} S_{n} G x_{n}}{\gamma_{n}+\delta_{n}}\right)
$$$$
+\frac{1-\alpha_{n+1}-\sigma_{n+1}-\theta_{n+1}}{1-\theta_{n+1}}
$$ 


$$
\begin{aligned}
& \times\left[\frac{\gamma_{n+1}}{\gamma_{n+1}+\delta_{n+1}}\left(B_{n+1} x_{n+1}-B_{n} x_{n}\right)\right. \\
& \quad+\left(\frac{\gamma_{n+1}}{\gamma_{n+1}+\delta_{n+1}}-\frac{\gamma_{n}}{\gamma_{n}+\delta_{n}}\right) B_{n} x_{n} \\
& \quad+\frac{\delta_{n+1}}{\gamma_{n+1}+\delta_{n+1}}\left(S_{n+1} G x_{n+1}-S_{n} G x_{n}\right) \\
& \left.\quad+\left(\frac{\delta_{n+1}}{\gamma_{n+1}+\delta_{n+1}}-\frac{\delta_{n}}{\gamma_{n}+\delta_{n}}\right) S_{n} G x_{n}\right],
\end{aligned}
$$

and hence

$$
\begin{aligned}
\| z_{n+1}- & z_{n} \| \\
\leq & \frac{\alpha_{n+1}}{1-\theta_{n+1}}\left\|f\left(x_{n+1}\right)-f\left(x_{n}\right)\right\| \\
& +\frac{\sigma_{n+1}}{1-\theta_{n+1}}\left\|G x_{n+1}-G x_{n}\right\| \\
& +\left|\frac{\alpha_{n+1}}{1-\theta_{n+1}}-\frac{\alpha_{n}}{1-\theta_{n}}\right|\left\|f\left(x_{n}\right)-\frac{\gamma_{n} B_{n} x_{n}+\delta_{n} S_{n} G x_{n}}{\gamma_{n}+\delta_{n}}\right\| \\
+ & \left|\frac{\sigma_{n+1}}{1-\theta_{n+1}}-\frac{\sigma_{n}}{1-\theta_{n}}\right|\left\|G x_{n}-\frac{\gamma_{n} B_{n} x_{n}+\delta_{n} S_{n} G x_{n}}{\gamma_{n}+\delta_{n}}\right\| \\
+ & \frac{1-\alpha_{n+1}-\sigma_{n+1}-\theta_{n+1}}{1-\theta_{n+1}} \\
\times & {\left[\frac{\gamma_{n+1}}{\gamma_{n+1}+\delta_{n+1}}\left\|B_{n+1} x_{n+1}-B_{n} x_{n}\right\|\right.} \\
& +\left|\frac{\gamma_{n+1}}{\gamma_{n+1}+\delta_{n+1}}-\frac{\gamma_{n}}{\gamma_{n}+\delta_{n}}\right|\left\|B_{n} x_{n}\right\| \\
& +\frac{\delta_{n+1}}{\gamma_{n+1}+\delta_{n+1}}\left\|S_{n+1} G x_{n+1}-S_{n} G x_{n}\right\| \\
& \left.+\left|\frac{\delta_{n+1}}{\gamma_{n+1}+\delta_{n+1}}-\frac{\delta_{n}}{\gamma_{n}+\delta_{n}}\right|\left\|S_{n} G x_{n}\right\|\right] .
\end{aligned}
$$

On the other hand, repeating the same arguments as those of (55) and (56) in the proof of Theorem 24, we can get

$$
\begin{gathered}
\left\|S_{n+1} G x_{n+1}-S_{n} G x_{n}\right\| \leq\left\|x_{n+1}-x_{n}\right\|+\left\|S_{n+1} G x_{n}-S_{n} G x_{n}\right\|, \\
\left\|B_{n+1} x_{n+1}-B_{n} x_{n}\right\| \leq\left\|x_{n+1}-x_{n}\right\|+M_{0} \prod_{i=0}^{n} \lambda_{i},
\end{gathered}
$$

for some constant $M_{0}>0$. Utilizing (140)-(141), we have $\left\|z_{n+1}-z_{n}\right\|$

$$
\begin{aligned}
\leq & \frac{\alpha_{n+1}}{1-\theta_{n+1}} \rho\left\|x_{n+1}-x_{n}\right\| \\
& +\frac{\sigma_{n+1}}{1-\theta_{n+1}}\left\|x_{n+1}-x_{n}\right\|
\end{aligned}
$$

$$
\begin{aligned}
& +\left|\frac{\alpha_{n+1}}{1-\theta_{n+1}}-\frac{\alpha_{n}}{1-\theta_{n}}\right| \\
& \times\left(\left\|f\left(x_{n}\right)\right\|+\left\|B_{n} x_{n}\right\|+\left\|S_{n} G x_{n}\right\|\right) \\
& +\left|\frac{\sigma_{n+1}}{1-\theta_{n+1}}-\frac{\sigma_{n}}{1-\theta_{n}}\right| \\
& \times\left(\left\|G x_{n}\right\|+\left\|B_{n} x_{n}\right\|+\left\|S_{n} G x_{n}\right\|\right) \\
& +\frac{1-\alpha_{n+1}-\sigma_{n+1}-\theta_{n+1}}{1-\theta_{n+1}} \\
& \times\left[\frac{\gamma_{n+1}}{\gamma_{n+1}+\delta_{n+1}}\left(\left\|x_{n+1}-x_{n}\right\|+M_{0} \prod_{i=0}^{n} \lambda_{i}\right)\right. \\
& \quad+\left|\frac{\gamma_{n+1}}{\gamma_{n+1}+\delta_{n+1}}-\frac{\gamma_{n}}{\gamma_{n}+\delta_{n}}\right|\left\|B_{n} x_{n}\right\| \\
& \quad+\frac{\delta_{n+1}}{\gamma_{n+1}+\delta_{n+1}}\left(\left\|x_{n+1}-x_{n}\right\|+\left\|S_{n+1} G x_{n}-S_{n} G x_{n}\right\|\right) \\
& \left.\quad+\left|\frac{\delta_{n+1}}{\gamma_{n+1}+\delta_{n+1}}-\frac{\delta_{n}}{\gamma_{n}+\delta_{n}}\right|\left\|S_{n} G x_{n}\right\|\right]
\end{aligned}
$$$$
=\frac{1-\alpha_{n+1}(1-\rho)-\theta_{n+1}}{1-\theta_{n+1}}\left\|x_{n+1}-x_{n}\right\|
$$$$
+\frac{1-\alpha_{n+1}-\sigma_{n+1}-\theta_{n+1}}{1-\theta_{n+1}}
$$$$
\times\left[\frac{\gamma_{n+1}}{\gamma_{n+1}+\delta_{n+1}} M_{0} \prod_{i=0}^{n} \lambda_{i}+\left|\frac{\delta_{n+1}}{\gamma_{n+1}+\delta_{n+1}}-\frac{\delta_{n}}{\gamma_{n}+\delta_{n}}\right|\right.
$$$$
\times\left(\left\|B_{n} x_{n}\right\|+\left\|S_{n} G x_{n}\right\|\right)
$$$$
\left.+\frac{\delta_{n+1}}{\gamma_{n+1}+\delta_{n+1}}\left\|S_{n+1} G x_{n}-S_{n} G x_{n}\right\|\right]
$$$$
+\left|\frac{\alpha_{n+1}}{1-\theta_{n+1}}-\frac{\alpha_{n}}{1-\theta_{n}}\right|
$$$$
\times\left(\left\|f\left(x_{n}\right)\right\|+\left\|B_{n} x_{n}\right\|+\left\|S_{n} G x_{n}\right\|\right)
$$$$
+\left|\frac{\sigma_{n+1}}{1-\theta_{n+1}}-\frac{\sigma_{n}}{1-\theta_{n}}\right|
$$$$
\times\left(\left\|G x_{n}\right\|+\left\|B_{n} x_{n}\right\|+\left\|S_{n} G x_{n}\right\|\right)
$$$$
\leq\left\|x_{n+1}-x_{n}\right\|
$$$$
+M\left(\prod_{i=0}^{n} \lambda_{i}+\left|\frac{\delta_{n+1}}{1-\beta_{n+1}}-\frac{\delta_{n}}{1-\beta_{n}}\right|\right.
$$$$
\left.+\left|\frac{\alpha_{n+1}}{1-\theta_{n+1}}-\frac{\alpha_{n}}{1-\theta_{n}}\right|+\left|\frac{\sigma_{n+1}}{1-\theta_{n+1}}-\frac{\sigma_{n}}{1-\theta_{n}}\right|\right)
$$

$+\left\|S_{n+1} G x_{n}-S_{n} G x_{n}\right\|$, 
where $\sup _{n \geq 0}\left\{\left\|f\left(x_{n}\right)\right\|+\left\|G x_{n}\right\|+\left\|B_{n} x_{n}\right\|+\left\|S_{n} G x_{n}\right\|+M_{0}\right\} \leq$ $M$ for some $M>0$. So, from (142), condition (iii), and the assumption on $\left\{S_{n}\right\}$ it follows that (noting that $0<\lambda_{i} \leq b<1$, for all $i \geq 0$ )

$$
\limsup _{n \rightarrow \infty}\left(\left\|z_{n+1}-z_{n}\right\|-\left\|x_{n+1}-x_{n}\right\|\right) \leq 0 .
$$

Consequently, by Lemma 20, we have

$$
\lim _{n \rightarrow \infty}\left\|z_{n}-x_{n}\right\|=0
$$

It follows from (137) and (138) that

$$
\lim _{n \rightarrow \infty}\left\|x_{n+1}-x_{n}\right\|=\lim _{n \rightarrow \infty}\left(1-\theta_{n}\right)\left\|z_{n}-x_{n}\right\|=0 .
$$

Next, we show that $\left\|x_{n}-G x_{n}\right\| \rightarrow 0$ as $n \rightarrow \infty$. Indeed, in terms of Lemma 11, from (130), we have

$$
\begin{aligned}
& \| y_{n}- p \|^{2} \\
& \leq \beta_{n}\left\|x_{n}-p\right\|^{2}+\gamma_{n}\left\|B_{n} x_{n}-p\right\|^{2} \\
&+\delta_{n}\left\|S_{n} G x_{n}-p\right\|^{2} \\
& \leq \beta_{n}\left\|x_{n}-p\right\|^{2}+\gamma_{n}\left\|x_{n}-p\right\|^{2}+\delta_{n}\left\|x_{n}-p\right\|^{2} \\
&=\left\|x_{n}-p\right\|^{2}, \\
& \| x_{n+1}-p \|^{2} \\
&=\| \alpha_{n}\left(x_{n}-p\right)+\sigma_{n}\left(G x_{n}-p\right)+\left(1-\alpha_{n}-\sigma_{n}\right)\left(y_{n}-p\right) \\
&+ \alpha_{n}\left(f\left(x_{n}\right)-x_{n}\right) \|^{2} \\
& \leq\left\|\alpha_{n}\left(x_{n}-p\right)+\sigma_{n}\left(G x_{n}-p\right)+\left(1-\alpha_{n}-\sigma_{n}\right)\left(y_{n}-p\right)\right\| \\
&+\left.\left\|\alpha_{n}\left(f\left(x_{n}\right)-x_{n}\right)\right\|\right]^{2} \\
&=\left\|\alpha_{n}\left(x_{n}-p\right)+\sigma_{n}\left(G x_{n}-p\right)+\left(1-\alpha_{n}-\sigma_{n}\right)\left(y_{n}-p\right)\right\|^{2} \\
&+\left\|\alpha_{n}\left(f\left(x_{n}\right)-x_{n}\right)\right\| \\
& \times {\left[2 \| \alpha_{n}\left(x_{n}-p\right)+\sigma_{n}\left(G x_{n}-p\right)\right.} \\
&+\left.+\left(1-\alpha_{n}-\sigma_{n}\right)\left(y_{n}-p\right)\|+\| \alpha_{n}\left(f\left(x_{n}\right)-x_{n}\right) \|\right] \\
&= \| \alpha_{n}\left(x_{n}-p\right)+\left(1-\alpha_{n}\right) \\
&+\left.\frac{\sigma_{n}}{1-\alpha_{n}}\left(G x_{n}-p\right)+\frac{1-\alpha_{n}-\sigma_{n}}{1-\alpha_{n}}\left(y_{n}-p\right)\right] \|^{2} \\
&\left.\left.+x_{n}\right)-x_{n}\right) \|
\end{aligned}
$$

$$
\begin{aligned}
\times & {\left[2 \| \alpha_{n}\left(x_{n}-p\right)+\sigma_{n}\left(G x_{n}-p\right)\right.} \\
& +\left(1-\alpha_{n}-\sigma_{n}\right)\left(y_{n}-p\right) \| \\
& \left.+\left\|\alpha_{n}\left(f\left(x_{n}\right)-x_{n}\right)\right\|\right] \\
\leq & \alpha_{n}\left\|x_{n}-p\right\|^{2} \\
+ & \left(1-\alpha_{n}\right)\left\|\frac{\sigma_{n}}{1-\alpha_{n}}\left(G x_{n}-p\right)+\frac{1-\alpha_{n}-\sigma_{n}}{1-\alpha_{n}}\left(y_{n}-p\right)\right\|^{2} \\
+ & \left\|\alpha_{n}\left(f\left(x_{n}\right)-x_{n}\right)\right\| \\
\times & {\left[2 \left(\alpha_{n}\left\|x_{n}-p\right\|+\sigma_{n}\left\|G x_{n}-p\right\|\right.\right.} \\
& \left.\left.+\left(1-\alpha_{n}-\sigma_{n}\right)\left\|y_{n}-p\right\|\right)+\left\|\alpha_{n}\left(f\left(x_{n}\right)-x_{n}\right)\right\|\right] \\
\leq & \alpha_{n}\left\|x_{n}-p\right\|^{2}+\left(1-\alpha_{n}\right) \\
\times & {\left[\frac{\sigma_{n}}{1-\alpha_{n}}\left\|G x_{n}-p\right\|^{2}+\frac{1-\alpha_{n}-\sigma_{n}}{1-\alpha_{n}}\left\|y_{n}-p\right\|^{2}\right.} \\
+ & \quad\left\|\alpha_{n}\left(f\left(x_{n}\right)-x_{n}\right)\right\|\left(2\left\|x_{n}-p\right\|+\left\|\alpha_{n}\left(f\left(x_{n}\right)-x_{n}\right)\right\|\right) . \\
& \left.\quad-\frac{\sigma_{n}\left(1-\alpha_{n}-\sigma_{n}\right)}{\left(1-\alpha_{n}\right)^{2}} g\left(\left\|G x_{n}-y_{n}\right\|\right)\right] \\
+ & \left\|\alpha_{n}\left(f\left(x_{n}\right)-x_{n}\right)\right\| \\
\times & +\left[2\left(\alpha_{n}\left\|x_{n}-p\right\|+\sigma_{n}\left\|x_{n}-p\right\|+\left(1-\alpha_{n}-\sigma_{n}\right)\left\|x_{n}-p\right\|\right)\right. \\
& +\| \alpha_{n}\left(f\left(x_{n}\right)-p\left\|^{2}+\left(1-\alpha_{n}\right)\right\|\right] \\
\times & \frac{\sigma_{n}}{1-\alpha_{n}}\left\|x_{n}-p\right\|^{2}+\frac{1-\alpha_{n}-\sigma_{n}}{1-\alpha_{n}}\left\|x_{n}-p\right\|^{2} \\
& \left.\left.\quad\left(1-\alpha_{n}\right)^{2}\right) g\left(\left\|G x_{n}-y_{n}\right\|\right)\right]
\end{aligned}
$$

Then, it immediately follows from $0 \leq \alpha_{n}+\sigma_{n} \leq 1-\rho$, for all $n \geq n_{0}$ that

$$
\begin{aligned}
& \rho \sigma_{n} g\left(\left\|G x_{n}-y_{n}\right\|\right) \\
& \leq \frac{\sigma_{n}\left(1-\alpha_{n}-\sigma_{n}\right)}{1-\alpha_{n}} g\left(\left\|G x_{n}-y_{n}\right\|\right) \\
& \leq\left\|x_{n}-p\right\|^{2}-\left\|x_{n+1}-p\right\|^{2} \\
& \quad+\left\|\alpha_{n}\left(f\left(x_{n}\right)-x_{n}\right)\right\|\left(2\left\|x_{n}-p\right\|+\left\|\alpha_{n}\left(f\left(x_{n}\right)-x_{n}\right)\right\|\right)
\end{aligned}
$$




$$
\begin{aligned}
\leq & \left(\left\|x_{n}-p\right\|+\left\|x_{n+1}-p\right\|\right)\left\|x_{n}-x_{n+1}\right\| \\
& +\left\|\alpha_{n}\left(f\left(x_{n}\right)-x_{n}\right)\right\| \\
& \times\left(2\left\|x_{n}-p\right\|+\left\|\alpha_{n}\left(f\left(x_{n}\right)-x_{n}\right)\right\|\right),
\end{aligned}
$$

for all $n \geq n_{0}$. Since $\left\|\alpha_{n}\left(f\left(x_{n}\right)-x_{n}\right)\right\| \rightarrow 0$ and $\left\{x_{n}\right\}$ is bounded, we deduce from (145) and condition (ii) that

$$
\lim _{n \rightarrow \infty} g\left(\left\|G x_{n}-y_{n}\right\|\right)=0 .
$$

Utilizing the properties of $g$, we have

$$
\lim _{n \rightarrow \infty}\left\|G x_{n}-y_{n}\right\|=0 .
$$

Also, from (130) we have

$$
\begin{aligned}
x_{n+1}- & x_{n} \\
= & \alpha_{n}\left(f\left(x_{n}\right)-x_{n}\right)+\sigma_{n}\left(G x_{n}-x_{n}\right) \\
& +\left(1-\alpha_{n}-\sigma_{n}\right)\left(y_{n}-x_{n}\right) \\
= & \alpha_{n}\left(f\left(x_{n}\right)-x_{n}\right)+\sigma_{n}\left(G x_{n}-y_{n}+y_{n}-x_{n}\right) \\
& +\left(1-\alpha_{n}-\sigma_{n}\right)\left(y_{n}-x_{n}\right) \\
= & \alpha_{n}\left(f\left(x_{n}\right)-x_{n}\right)+\sigma_{n}\left(G x_{n}-y_{n}\right) \\
& +\left(1-\alpha_{n}\right)\left(y_{n}-x_{n}\right),
\end{aligned}
$$

which hence leads to

$$
\begin{aligned}
\rho \| & y_{n}-x_{n} \| \\
& \leq\left(1-\alpha_{n}-\sigma_{n}\right)\left\|y_{n}-x_{n}\right\| \\
& \leq\left(1-\alpha_{n}\right)\left\|y_{n}-x_{n}\right\| \\
& =\left\|x_{n+1}-x_{n}-\alpha_{n}\left(f\left(x_{n}\right)-x_{n}\right)-\sigma_{n}\left(G x_{n}-y_{n}\right)\right\| \\
& \leq\left\|x_{n+1}-x_{n}\right\|+\left\|\alpha_{n}\left(f\left(x_{n}\right)-x_{n}\right)\right\|+\sigma_{n}\left\|G x_{n}-y_{n}\right\| \\
& \leq\left\|x_{n+1}-x_{n}\right\|+\left\|\alpha_{n}\left(f\left(x_{n}\right)-x_{n}\right)\right\|+\left\|G x_{n}-y_{n}\right\| .
\end{aligned}
$$

So, it is easy to see from (145), (149), and $\left\|\alpha_{n}\left(f\left(x_{n}\right)-x_{n}\right)\right\| \rightarrow$ 0 that

$$
\lim _{n \rightarrow \infty}\left\|y_{n}-x_{n}\right\|=0
$$

We note that

$$
\left\|G x_{n}-x_{n}\right\| \leq\left\|G x_{n}-y_{n}\right\|+\left\|y_{n}-x_{n}\right\| .
$$

Therefore, from (149) and (152) it follows that

$$
\lim _{n \rightarrow \infty}\left\|G x_{n}-x_{n}\right\|=0 \text {. }
$$

Repeating the same arguments as those of (86), (89), and (91) in the proof of Theorem 24, we can obtain

$$
\begin{aligned}
\lim _{n \rightarrow \infty}\left\|x_{n}-B_{n} x_{n}\right\| & =\lim _{n \rightarrow \infty}\left\|S_{n} G x_{n}-B_{n} x_{n}\right\| \\
& =\lim _{n \rightarrow \infty}\left\|x_{n}-S x_{n}\right\|=0 .
\end{aligned}
$$

Suppose that $\beta_{n} \equiv \beta$ for some fixed $\beta \in(0,1)$ such that $\beta+$ $\gamma_{n}+\delta_{n}=1$ for all $n \geq 0$. Define a mapping $V x=\left(1-\theta_{1}-\right.$ $\left.\theta_{2}\right) S x+\theta_{1} B x+\theta_{2} G x$, where $\theta_{1}, \theta_{2} \in(0,1)$ are two constants with $\theta_{1}+\theta_{2}<1$. Then, by Lemmas 14 and 17 , we have that $\operatorname{Fix}(V)=\operatorname{Fix}(S) \cap \operatorname{Fix}(B) \cap \operatorname{Fix}(G)=F$. For each $k \geq 1$, let $\left\{p_{k}\right\}$ be a unique element of $C$ such that

$$
p_{k}=\frac{1}{k} f\left(p_{k}\right)+\left(1-\frac{1}{k}\right) V p_{k} .
$$

From Lemma 13, we conclude that $p_{k} \rightarrow q \in \operatorname{Fix}(V)=F$ as $k \rightarrow \infty$. Observe that for every $n, k$

$$
\begin{aligned}
\| y_{n}- & B p_{k} \| \\
\leq & \beta\left\|x_{n}-B p_{k}\right\|+\gamma_{n}\left\|B_{n} x_{n}-B p_{k}\right\| \\
& +\delta_{n}\left(\left\|S_{n} G x_{n}-B_{n} x_{n}\right\|+\left\|B_{n} x_{n}-B p_{k}\right\|\right) \\
= & \beta\left\|x_{n}-B p_{k}\right\|+(1-\beta)\left\|B_{n} x_{n}-B p_{k}\right\| \\
& +\delta_{n}\left\|S_{n} G x_{n}-B_{n} x_{n}\right\|,
\end{aligned}
$$

and hence

$$
\begin{aligned}
& \left\|x_{n+1}-B p_{k}\right\| \\
& \leq \alpha_{n}\left\|f\left(x_{n}\right)-B p_{k}\right\|+\sigma_{n}\left\|G x_{n}-B p_{k}\right\| \\
& +\left(1-\alpha_{n}-\sigma_{n}\right)\left\|y_{n}-B p_{k}\right\| \\
& \leq \alpha_{n}\left(\left\|f\left(x_{n}\right)-x_{n}\right\|+\left\|x_{n}-B p_{k}\right\|\right) \\
& +\sigma_{n}\left(\left\|G x_{n}-x_{n}\right\|+\left\|x_{n}-B p_{k}\right\|\right)+\left(1-\alpha_{n}-\sigma_{n}\right) \\
& \times\left[\beta\left\|x_{n}-B p_{k}\right\|+(1-\beta)\left\|B_{n} x_{n}-B p_{k}\right\|\right. \\
& \left.+\delta_{n}\left\|S_{n} G x_{n}-B_{n} x_{n}\right\|\right] \\
& \leq \alpha_{n}\left(\left\|f\left(x_{n}\right)-x_{n}\right\|+\left\|x_{n}-B p_{k}\right\|\right) \\
& +\sigma_{n}\left(\left\|G x_{n}-x_{n}\right\|+\left\|x_{n}-B p_{k}\right\|\right)+\left(1-\alpha_{n}-\sigma_{n}\right) \\
& \times\left[\beta\left\|x_{n}-B p_{k}\right\|\right. \\
& +(1-\beta)\left(\left\|B_{n} x_{n}-B_{n} p_{k}\right\|+\left\|B_{n} p_{k}-B p_{k}\right\|\right) \\
& \left.+\delta_{n}\left\|S_{n} G x_{n}-B_{n} x_{n}\right\|\right] \\
& \leq \alpha_{n}\left(\left\|f\left(x_{n}\right)-x_{n}\right\|+\left\|x_{n}-B p_{k}\right\|\right) \\
& +\sigma_{n}\left(\left\|G x_{n}-x_{n}\right\|+\left\|x_{n}-B p_{k}\right\|\right)+\left(1-\alpha_{n}-\sigma_{n}\right) \\
& \times\left[\beta\left\|x_{n}-B p_{k}\right\|+(1-\beta)\left(\left\|x_{n}-p_{k}\right\|+\left\|B_{n} p_{k}-B p_{k}\right\|\right)\right. \\
& \left.+\delta_{n}\left\|S_{n} G x_{n}-B_{n} x_{n}\right\|\right] \\
& =\alpha_{n}\left\|f\left(x_{n}\right)-x_{n}\right\|+\sigma_{n}\left\|G x_{n}-x_{n}\right\| \\
& +\left[\beta+\left(\alpha_{n}+\sigma_{n}\right)(1-\beta)\right]\left\|x_{n}-B p_{k}\right\| \\
& +\left(1-\alpha_{n}-\sigma_{n}\right)(1-\beta)\left\|x_{n}-p_{k}\right\|+\left(1-\alpha_{n}-\sigma_{n}\right) \\
& \times\left[(1-\beta)\left\|B_{n} p_{k}-B p_{k}\right\|+\delta_{n}\left\|S_{n} G x_{n}-B_{n} x_{n}\right\|\right]
\end{aligned}
$$




$$
\begin{aligned}
\leq & \alpha_{n}\left\|f\left(x_{n}\right)-x_{n}\right\|+\sigma_{n}\left\|G x_{n}-x_{n}\right\| \\
& +\left[\beta+\left(\alpha_{n}+\sigma_{n}\right)(1-\beta)\right] \\
& \times\left(\left\|x_{n}-x_{n+1}\right\|+\left\|x_{n+1}-B p_{k}\right\|\right)+\left(1-\alpha_{n}-\sigma_{n}\right)(1-\beta) \\
& \times\left(\left\|x_{n}-x_{n+1}\right\|+\left\|x_{n+1}-p_{k}\right\|\right)+\left(1-\alpha_{n}-\sigma_{n}\right) \\
& \times\left[(1-\beta)\left\|B_{n} p_{k}-B p_{k}\right\|+\delta_{n}\left\|S_{n} G x_{n}-B_{n} x_{n}\right\|\right] \\
= & \alpha_{n}\left\|f\left(x_{n}\right)-x_{n}\right\|+\sigma_{n}\left\|G x_{n}-x_{n}\right\| \\
& +\left[\beta+\left(\alpha_{n}+\sigma_{n}\right)(1-\beta)\right]\left\|x_{n+1}-B p_{k}\right\| \\
& +\left(1-\alpha_{n}-\sigma_{n}\right)(1-\beta)\left\|x_{n+1}-p_{k}\right\|+\left(1-\alpha_{n}-\sigma_{n}\right) \\
& \times\left[(1-\beta)\left\|B_{n} p_{k}-B p_{k}\right\|+\delta_{n}\left\|S_{n} G x_{n}-B_{n} x_{n}\right\|\right] \\
& +\left\|x_{n}-x_{n+1}\right\| .
\end{aligned}
$$

So, it immediately follows from $0 \leq \alpha_{n} \leq 1-\rho$, for all $n \geq n_{0}$ that

$$
\begin{aligned}
& \left\|x_{n+1}-B p_{k}\right\| \\
& \leq\left\|x_{n+1}-p_{k}\right\|+\left\|B_{n} p_{k}-B p_{k}\right\| \\
& +\frac{1}{\left(1-\alpha_{n}-\sigma_{n}\right)(1-\beta)} \\
& \quad \times\left(\left\|\alpha_{n}\left(x_{n}-f\left(x_{n}\right)\right)\right\|+\sigma_{n}\left\|G x_{n}-x_{n}\right\|\right. \\
& \left.\quad+\left\|x_{n}-x_{n+1}\right\|\right)+\frac{\delta_{n}}{1-\beta}\left\|S_{n} G x_{n}-B_{n} x_{n}\right\| \\
& \leq\left\|x_{n+1}-p_{k}\right\|+\left\|B_{n} p_{k}-B p_{k}\right\| \\
& +\left\|S_{n} G x_{n}-B_{n} x_{n}\right\|+\frac{1}{\rho(1-\beta)} \\
& \quad \times\left(\left\|\alpha_{n}\left(x_{n}-f\left(x_{n}\right)\right)\right\|\right. \\
& \left.\quad+\left\|G x_{n}-x_{n}\right\|+\left\|x_{n}-x_{n+1}\right\|\right) \\
& =\left\|x_{n+1}-p_{k}\right\|+\tau_{n}, \quad \forall n \geq n_{0},
\end{aligned}
$$

where $\theta_{n}=\left\|B_{n} p_{k}-B p_{k}\right\|+\left\|S_{n} G x_{n}-B_{n} x_{n}\right\|+1 /(\rho(1-$ $\beta))\left(\left\|\alpha_{n}\left(x_{n}-f\left(x_{n}\right)\right)\right\|+\left\|G x_{n}-x_{n}\right\|+\left\|x_{n}-x_{n+1}\right\|\right)$. Since $\lim _{n \rightarrow \infty}\left\|B_{n} p_{k}-B p_{k}\right\|=\lim _{n \rightarrow \infty}\left\|S_{n} G x_{n}-B_{n} x_{n}\right\|=$ $\lim _{n \rightarrow \infty}\left\|\alpha_{n}\left(x_{n}-f\left(x_{n}\right)\right)\right\|=\lim _{n \rightarrow \infty}\left\|G x_{n}-x_{n}\right\|=$ $\lim _{n \rightarrow \infty}\left\|x_{n}-x_{n+1}\right\|=0$, we know that $\tau_{n} \rightarrow 0$ as $n \rightarrow \infty$.

From (159), we obtain

$$
\begin{array}{r}
\left\|x_{n+1}-B p_{k}\right\|^{2} \leq\left\|x_{n+1}-p_{k}\right\|^{2}+\tau_{n}\left(2\left\|x_{n+1}-p_{k}\right\|+\tau_{n}\right), \\
\forall n \geq n_{0} .
\end{array}
$$

For any Banach limit $\mu$, from (160) we derive

$$
\begin{aligned}
\mu_{n}\left\|x_{n}-B p_{k}\right\|^{2} & =\mu_{n}\left\|x_{n+1}-B p_{k}\right\|^{2} \\
& \leq \mu_{n}\left\|x_{n+1}-p_{k}\right\|^{2}=\mu_{n}\left\|x_{n}-p_{k}\right\|^{2} .
\end{aligned}
$$

Repeating the same arguments as those of (99), in the proof of Theorem 24, we can get

$$
\begin{gathered}
\mu_{n}\left\|x_{n}-G p_{k}\right\|^{2} \leq \mu_{n}\left\|x_{n}-p_{k}\right\|^{2}, \\
\mu_{n}\left\|x_{n}-S p_{k}\right\|^{2} \leq \mu_{n}\left\|x_{n}-p_{k}\right\|^{2} .
\end{gathered}
$$

Utilizing (161) and (162), we deduce that

$$
\begin{aligned}
\mu_{n} \| & x_{n}-V p_{k} \|^{2} \\
& \leq\left(1-\theta_{1}-\theta_{2}\right) \mu_{n}\left\|x_{n}-S p_{k}\right\|^{2} \\
& \quad+\theta_{1} \mu_{n}\left\|x_{n}-B p_{k}\right\|^{2}+\theta_{2} \mu_{n}\left\|x_{n}-G p_{k}\right\|^{2} \\
& \leq \mu_{n}\left\|x_{n}-p_{k}\right\|^{2} .
\end{aligned}
$$

Also, observe that

$$
\left(1-\frac{1}{k}\right)\left(x_{n}-V p_{k}\right)=x_{n}-p_{k}-\frac{1}{k}\left(x_{n}-f\left(p_{k}\right)\right) .
$$

Repeating the same arguments as those of (106) in the proof of Theorem 24, we can get

$$
\frac{1}{2 k} \mu_{n}\left\|x_{n}-p_{k}\right\|^{2} \geq \mu_{n}\left\langle f\left(p_{k}\right)-p_{k}, J\left(x_{n}-p_{k}\right)\right\rangle .
$$

Since $p_{k} \rightarrow q \in \operatorname{Fix}(V)=F$ as $k \rightarrow \infty$, by the uniform Gateaux differentiability of the norm of $X$, we have

$$
\mu_{n}\left\langle f(q)-q, J\left(x_{n}-q\right)\right\rangle \leq 0 .
$$

On the other hand, from (135) and the norm-to-weak* uniform continuity of $J$ on bounded subsets of $X$, it follows that

$\lim _{n \rightarrow \infty}\left|\left\langle f(q)-q, J\left(x_{n+1}-q\right)\right\rangle-\left\langle f(q)-q, J\left(x_{n}-q\right)\right\rangle\right|=0$.

So, utilizing Lemma 18, we deduce from (166) and (167) that

$$
\limsup _{n \rightarrow \infty}\left\langle f(q)-q, J\left(x_{n}-q\right)\right\rangle \leq 0
$$

which, together with (135) and the norm-to-norm uniform continuity of $J$ on bounded subsets of $X$, implies that

$$
\limsup _{n \rightarrow \infty}\left\langle f(q)-q, J\left(x_{n+1}-q\right)\right\rangle \leq 0 .
$$


Finally, let us show that $x_{n} \rightarrow q$ as $n \rightarrow \infty$. Utilizing Lemma 8 (i), from (130) and the convexity of $\|\cdot\|^{2}$, we get

$$
\begin{aligned}
&\left\|y_{n}-q\right\|^{2} \leq \beta_{n}\left\|x_{n}-q\right\|^{2}+\gamma_{n}\left\|B_{n} x_{n}-q\right\|^{2} \\
&+\delta_{n}\left\|S_{n} G x_{n}-q\right\|^{2} \leq\left\|x_{n}-q\right\|^{2}, \\
&\left\|x_{n+1}-q\right\|^{2} \\
&=\| \alpha_{n}\left(f\left(x_{n}\right)-f(q)\right)+\sigma_{n}\left(G x_{n}-q\right) \\
&+\left(1-\alpha_{n}-\sigma_{n}\right)\left(y_{n}-q\right)+\alpha_{n}(f(q)-q) \|^{2} \\
& \leq \| \alpha_{n}\left(f\left(x_{n}\right)-f(q)\right)+\sigma_{n}\left(G x_{n}-q\right) \\
&+\left(1-\alpha_{n}-\sigma_{n}\right)\left(y_{n}-q\right) \|^{2} \\
&+2 \alpha_{n}\left\langle f(q)-q, J\left(x_{n+1}-q\right)\right\rangle \\
& \leq \alpha_{n}\left\|f\left(x_{n}\right)-f(q)\right\|^{2}+\sigma_{n}\left\|G x_{n}-q\right\|^{2} \\
&+\left(1-\alpha_{n}-\sigma_{n}\right)\left\|y_{n}-q\right\|^{2} \\
&+2 \alpha_{n}\left\langle f(q)-q, J\left(x_{n+1}-q\right)\right\rangle \\
& \leq \alpha_{n} \rho\left\|x_{n}-q\right\|^{2}+\sigma_{n}\left\|x_{n}-q\right\|^{2} \\
&+\left(1-\alpha_{n}-\sigma_{n}\right)\left\|x_{n}-q\right\|^{2} \\
&+2 \alpha_{n}\left\langle f(q)-q, J\left(x_{n+1}-q\right)\right\rangle \\
&=\left(1-\alpha_{n}(1-\rho)\right)\left\|x_{n}-q\right\|^{2} \\
&+2 \alpha_{n}\left\langle f(q)-q, J\left(x_{n+1}-q\right)\right\rangle \\
&=\left(1-\alpha_{n}(1-\rho)\right)\left\|x_{n}-q\right\|^{2} \\
&+\alpha_{n}(1-\rho) \frac{2\left\langle f(q)-q, J\left(x_{n+1}-q\right)\right\rangle}{1-\rho} .
\end{aligned}
$$

Applying Lemma 7 to (171), we obtain that $x_{n} \rightarrow q$ as $n \rightarrow$ $\infty$.

Conversely, if $x_{n} \rightarrow q \in F$ as $n \rightarrow \infty$, then from (130) it follows that

$$
\begin{aligned}
\left\|y_{n}-q\right\| \leq & \beta_{n}\left\|x_{n}-q\right\|+\gamma_{n}\left\|B_{n} x_{n}-q\right\| \\
& +\delta_{n}\left\|S_{n} G x_{n}-q\right\| \leq\left\|x_{n}-q\right\| \longrightarrow 0
\end{aligned}
$$

as $n \rightarrow \infty$; that is, $y_{n} \rightarrow q$. Again from (130) we obtain that

$$
\begin{aligned}
\| \alpha_{n} & \left(f\left(x_{n}\right)-x_{n}\right) \| \\
= & \left\|x_{n+1}-x_{n}-\sigma_{n}\left(G x_{n}-x_{n}\right)-\left(1-\alpha_{n}-\sigma_{n}\right)\left(y_{n}-x_{n}\right)\right\| \\
\leq & \left\|x_{n+1}-x_{n}\right\|+\sigma_{n}\left\|G x_{n}-x_{n}\right\|+\left(1-\alpha_{n}-\sigma_{n}\right)\left\|y_{n}-x_{n}\right\| \\
\leq & \left\|x_{n+1}-q\right\|+\left\|x_{n}-q\right\|+\sigma_{n}\left(\left\|G x_{n}-q\right\|+\left\|x_{n}-q\right\|\right) \\
& +\left(1-\alpha_{n}-\sigma_{n}\right)\left(\left\|y_{n}-q\right\|+\left\|x_{n}-q\right\|\right)
\end{aligned}
$$

$$
\begin{aligned}
\leq & \left\|x_{n+1}-q\right\|+\left\|x_{n}-q\right\|+\sigma_{n}\left(\left\|x_{n}-q\right\|+\left\|x_{n}-q\right\|\right) \\
& +\left(1-\alpha_{n}-\sigma_{n}\right)\left(\left\|y_{n}-q\right\|+\left\|x_{n}-q\right\|\right) \\
\leq & \left\|x_{n+1}-q\right\|+3\left\|x_{n}-q\right\|+\left\|y_{n}-q\right\| .
\end{aligned}
$$

Since $x_{n} \rightarrow q$ and $y_{n} \rightarrow q$, we get $\alpha_{n}\left(f\left(x_{n}\right)-x_{n}\right) \rightarrow 0$. This completes the proof.

Corollary 29. Let $C$ be a nonempty closed convex subset of a uniformly convex Banach space $X$ which has a uniformly Gateaux differentiable norm. Let $\Pi_{C}$ be a sunny nonexpansive retraction from $X$ onto $C$. Let $\left\{\rho_{n}\right\}_{n=0}^{\infty}$ be a sequence of positive numbers in $(0, b]$ for some $b \in(0,1)$ and $A_{i}: C \rightarrow X \xi_{i}$ strictly pseudocontractive and $\widehat{\alpha}_{i}$-strongly accretive with $\xi_{i}+$ $\widehat{\alpha}_{i} \geq 1$ for each $i=0,1, \ldots$. Define a mapping $G_{i}: C \rightarrow C$ by $\Pi_{C}\left(I-\lambda_{i} A_{i}\right) x=G_{i} x$ for all $x \in C$ and $i=0,1, \ldots$, where $1-\left(\xi_{i} /\left(1+\xi_{i}\right)\right)\left(1-\sqrt{\left(1-\widehat{\alpha}_{i}\right) / \xi_{i}}\right) \leq \lambda_{i} \leq 1$ for all $i=0,1, \ldots$ Let $B_{n}: C \rightarrow C$ be the $W$-mapping generated by $G_{n}, G_{n-1}, \ldots, G_{0}$ and $\rho_{n}, \rho_{n-1}, \ldots, \rho_{0}$. Let $V: C \rightarrow C$ be a self-mapping such that $I-V: C \rightarrow X$ is $\lambda$-strictly pseudocontractive and $\alpha$ strongly accretive with $\alpha+\lambda \geq 1$. Let $f: C \rightarrow C$ be a contraction with coefficient $\rho \in(0,1)$. Let $\left\{S_{i}\right\}_{i=0}^{\infty}$ be a countable family of nonexpansive mappings of $C$ into itself such that $F=$ $\left(\bigcap_{i=0}^{\infty} \operatorname{Fix}\left(S_{i}\right)\right) \cap \operatorname{Fix}(V) \cap\left(\bigcap_{i=0}^{\infty} \operatorname{VI}\left(C, A_{i}\right)\right) \neq \emptyset$. For arbitrarily given $x_{0} \in C$, let $\left\{x_{n}\right\}$ be the sequence generated by

$$
\begin{aligned}
& y_{n}=\beta_{n} x_{n}+\gamma_{n} B_{n} x_{n}+\delta_{n} S_{n}((1-l) I+l V) x_{n}, \\
& x_{n+1}=\alpha_{n} f\left(x_{n}\right)+\sigma_{n}((1-l) I+l V) x_{n}+\left(1-\alpha_{n}-\sigma_{n}\right) y_{n},
\end{aligned}
$$

where $1-(\lambda /(1+\lambda))(1-\sqrt{(1-\alpha) / \lambda}) \leq l \leq 1$ and $\left\{\sigma_{n}\right\}$, $\left\{\alpha_{n}\right\},\left\{\beta_{n}\right\},\left\{\gamma_{n}\right\}$, and $\left\{\delta_{n}\right\}$ are the sequences in $[0,1]$ such that $\beta_{n}+\gamma_{n}+\delta_{n}=1$ and $\alpha_{n}+\sigma_{n} \leq 1$ for all $n \geq 0$. Suppose that the following conditions hold:

(i) $\sum_{n=0}^{\infty} \alpha_{n}=\infty$ and $0 \leq \alpha_{n}+\sigma_{n} \leq 1-\rho$, for all $n \geq n_{0}$ for some integer $n_{0} \geq 0$;

(ii) $\liminf _{n \rightarrow \infty} \sigma_{n}>0, \liminf _{n \rightarrow \infty} \gamma_{n}>0$ and $\liminf _{n \rightarrow \infty} \delta_{n}>0$;

(iii) $\lim _{n \rightarrow \infty}\left(\mid \alpha_{n+1} /\left(1-\left(1-\alpha_{n+1}-\sigma_{n+1}\right) \beta_{n+1}\right)-\alpha_{n} /(1-(1-\right.$ $\left.\left.\alpha_{n}-\sigma_{n}\right) \beta_{n}\right)|+| \sigma_{n+1} /\left(1-\left(1-\alpha_{n+1}-\sigma_{n+1}\right) \beta_{n+1}\right)-\sigma_{n} /(1-$ $\left.\left.\left(1-\alpha_{n}-\sigma_{n}\right) \beta_{n}\right)|+| \delta_{n+1} /\left(1-\beta_{n+1}\right)-\delta_{n} /\left(1-\beta_{n}\right) \mid\right)=0 ;$

(iv) $0<\liminf _{n \rightarrow \infty} \beta_{n} \leq \lim \sup _{n \rightarrow \infty} \beta_{n}<1$.

Assume that $\sum_{n=0}^{\infty} \sup _{x \in D}\left\|S_{n+1} x-S_{n} x\right\|<\infty$ for any bounded subset $D$ of $C$ and let $S$ be a mapping of $C$ into itself defined by $S x=\lim _{n \rightarrow \infty} S_{n} x$ for all $x \in C$ and suppose that $\operatorname{Fix}(S)=$ $\bigcap_{i=0}^{\infty} \operatorname{Fix}\left(S_{i}\right)$. Then there hold the following:

(I) $\lim _{n \rightarrow \infty}\left\|x_{n+1}-x_{n}\right\|=0$;

(II) $x_{n} \rightarrow q \Leftrightarrow \alpha_{n}\left(f\left(x_{n}\right)-x_{n}\right) \rightarrow 0$ provided $\beta_{n} \equiv \beta$ for some fixed $\beta \in(0,1)$, where $q \in F$ solves the following VIP

$$
\langle q-f(q), J(q-p)\rangle \leq 0, \quad \forall p \in F .
$$


Proof. In Theorem 28, we put $B_{1}=I-V, B_{2}=0$, and $\mu_{1}=l$, where $1-(\lambda /(1+\lambda))(1-\sqrt{(1-\alpha) / \lambda}) \leq l \leq 1$. Then, GSVI (13) is equivalent to the VIP of finding $x^{*} \in C$ such that

$$
\left\langle B_{1} x^{*}, J\left(x-x^{*}\right)\right\rangle \geq 0, \quad \forall x \in C .
$$

In this case, $B_{1}: C \rightarrow X$ is $\lambda$-strictly pseudocontractive and $\alpha$-strongly accretive. Repeating the same arguments as those in the proof of Corollary 25, we can infer that $\operatorname{Fix}(V)=\operatorname{VI}\left(C, B_{1}\right)$. Accordingly, $F=\bigcap_{i=0}^{\infty} \operatorname{Fix}\left(S_{i}\right) \cap \Omega \cap$ $\left(\bigcap_{i=0}^{\infty} \operatorname{VI}\left(C, A_{i}\right)\right)=\bigcap_{i=0}^{\infty} \operatorname{Fix}\left(S_{i}\right) \cap \operatorname{Fix}(V) \cap\left(\bigcap_{i=0}^{\infty} \operatorname{VI}\left(C, A_{i}\right)\right)$, and

$$
G x_{n}=((1-l) I+l V) x_{n}, \quad \forall n \geq 0 .
$$

So, scheme (130) reduces to (174). Therefore, the desired result follows from Theorem 31 .

Remark 30. Our Theorems 24 and 28 improve, extend, supplement and develop Ceng and Yao's [10, Theorem 3.2], Cai and Bu's [11, Theorem 3.1], Kangtunyakarn's [38, Theorem 3.1], and Ceng and Yao's [8, Theorem 3.1], in the following aspects.

(i) The problem of finding a point $q \in\left(\bigcap_{i=0}^{\infty} \operatorname{Fix}\left(S_{i}\right)\right) \cap$ $\Omega \cap\left(\bigcap_{i=0}^{\infty} \operatorname{VI}\left(C, A_{i}\right)\right)$ in our Theorems 24 and 28 is more general and more subtle than every one of the problem of finding a point $q \in \bigcap_{i=0}^{\infty} \operatorname{Fix}\left(T_{i}\right)$ in $[10$, Theorem 3.2], the problem of finding a point $q \in$ $\bigcap_{i=1}^{\infty} \operatorname{Fix}\left(T_{i}\right) \cap \Omega$ in [11, Theorem 3.1], the problem of finding a point $q \in \operatorname{Fix}(S) \cap \operatorname{Fix}(V) \cap\left(\bigcap_{i=1}^{N} \operatorname{VI}\left(C, A_{i}\right)\right)$ in [38, Theorem 3.1], and the problem of finding a point $q \in \operatorname{Fix}(T)$ in [8, Theorem 3.1].

(ii) The iterative scheme in [8, Theorem 3.1] is extended to develop the iterative schemes (42) and (130) in our Theorems 24 and 28 by virtue of the iterative schemes of [11, Theorem 3.1] and [10, Theorems 3.2]. The iterative schemes (42) and (130) in our Theorems 24 and 28 are more advantageous and more flexible than the iterative scheme of [8, Theorem 3.1] because they can be applied to solving three problems (i.e., GSVI (13), fixed point problem and infinitely many VIPs), and involve several parameter sequences $\left\{\alpha_{n}\right\}$, $\left\{\beta_{n}\right\},\left\{\gamma_{n}\right\},\left\{\delta_{n}\right\}$, (and $\left\{\sigma_{n}\right\}$ ).

(iii) Our Theorems 24 and 28 extend and generalize Ceng and Yao [8, Theorem 3.1] from a nonexpansive mapping to a countable family of nonexpansive mappings, and Ceng and Yao's [10, Theorems 3.2], to the setting of the GSVI (13) and infinitely many VIPs, Kangtunyakarn [38, Theorem 3.1], from finitely many VIPs to infinitely many VIPs, from a nonexpansive mapping to a countable family of nonexpansive mappings and from a strict pseudocontraction to the GSVI (13). In the meantime, our Theorems 24 and 28 extend and generalize Cai and Bu's [11, Theorem 3.1], to the setting of infinitely many VIPs.

(iv) The iterative schemes (42) and (130) in our Theorems 24 and 28 are very different from every one in [10, Theorem 3.2], [11, Theorem 3.1], [38, Theorem 3.1], and [8, Theorem 3.1] because the mappings $G$ and $T_{n}$ in [11, Theorem 3.1] and the mapping $T$ in $[8$, Theorem 3.1] are replaced with the same composite mapping $S_{n} G$ in the iterative schemes (42) and (130) and the mapping $W_{n}$ in [10, Theorem 3.2] is replaced with $B_{n}$.

(v) Cai and Bu's proof in [11, Theorem 3.1] depends on the argument techniques in [14], the inequality in 2uniformly smooth Banach spaces (see Lemma 4), and the inequality in smooth and uniform convex Banach spaces (see Proposition 6). Because the composite mapping $S_{n} G$ appears in the iterative scheme (42) of our Theorem 24, the proof of our Theorem 24 depends on the argument techniques in [14], the inequality in 2-uniformly smooth Banach spaces (see Lemma 4), the inequality in smooth and uniform convex Banach spaces (see Proposition 6), the inequality in uniform convex Banach spaces (see Lemma 15 in Section 2 of this paper), and the properties of the $W$-mapping and the Banach limit (see Lemmas 16-18 in Section 2 of this paper). However, the proof of our Theorem 28 does not depend on the argument techniques in [14], the inequality in 2-uniformly smooth Banach spaces (see Lemma 4), and the inequality in smooth and uniform convex Banach spaces (see Proposition 6). It depends on only the inequality in uniform convex Banach spaces (see Lemma 15 in Section 2 of this paper) and the properties of the $W$-mapping and the Banach limit (see Lemmas 16-18 in Section 2 of this paper).

(vi) The assumption of the uniformly convex and 2uniformly smooth Banach space $X$ in [11, Theorem 3.1] is weakened to the one of the uniformly convex Banach space $X$ having a uniformly Gateaux differentiable norm in our Theorem 28. Moreover, the assumption of the uniformly smooth Banach space $X$ in [8, Theorem 3.1] is replaced with the one of the uniformly convex Banach space $X$ having a uniformly Gateaux differentiable norm in our Theorem 28. It is worth emphasizing that there is no assumption on the convergence of parameter sequences $\left\{\alpha_{n}\right\},\left\{\beta_{n}\right\},\left\{\gamma_{n}\right\}$, and $\left\{\delta_{n}\right\}$ (and $\left\{\sigma_{n}\right\}$ ) to zero in our Theorems 24 and 28 .

\section{Relaxed Mann Iterations and Their Convergence Criteria}

In this section, we introduce our relaxed Mann iteration algorithms in real smooth and uniformly convex Banach spaces and present their convergence criteria.

Theorem 31. Let $C$ be a nonempty closed convex subset of a uniformly convex and 2-uniformly smooth Banach space $X$. Let $\Pi_{C}$ be a sunny nonexpansive retraction from $X$ onto $C$. Let $\left\{\rho_{n}\right\}_{n=0}^{\infty}$ be a sequence of positive numbers in $(0, b]$ for some $b \in(0,1)$ and $A_{i}: C \rightarrow X$ an $\widehat{\alpha}_{i}$-inverse strongly accretive mapping for each $i=0,1, \ldots$. Define a mapping $G_{i}: C \rightarrow C$ by $\Pi_{C}\left(I-\lambda_{i} A_{i}\right) x=G_{i} x$ for all $x \in C$ and $i=0,1, \ldots$, 
where $\lambda_{i} \in\left(0, \widehat{\alpha}_{i} / \kappa^{2}\right]$ and $\kappa$ is the 2 -uniformly smooth constant of $X$. Let $B_{n}: C \rightarrow C$ be the $W$-mapping generated by $G_{n}, G_{n-1}, \ldots, G_{0}$ and $\rho_{n}, \rho_{n-1}, \ldots, \rho_{0}$. Let the mapping $B_{i}$ : $C \rightarrow X$ be $\widehat{\beta}_{i}$-inverse strongly accretive for $i=1,2$. Let $f$ : $C \rightarrow C$ be a contraction with coefficient $\rho \in(0,1)$. Let $\left\{S_{i}\right\}_{i=0}^{\infty}$ be a countable family of nonexpansive mappings of $C$ into itself such that $F=\left(\bigcap_{i=0}^{\infty} \operatorname{Fix}\left(S_{i}\right)\right) \cap \Omega \cap\left(\bigcap_{i=0}^{\infty} \operatorname{VI}\left(C, A_{i}\right)\right) \neq \emptyset$, where $\Omega$ is the fixed point set of the mapping $G=\Pi_{C}\left(I-\mu_{1} B_{1}\right) \Pi_{C}(I-$ $\left.\mu_{2} B_{2}\right)$ with $0<\mu_{i}<\widehat{\beta}_{i} / \kappa^{2}$ for $i=1,2$. For arbitrarily given $x_{0} \in C$, let $\left\{x_{n}\right\}$ be the sequence generated by

$$
x_{n+1}=\alpha_{n} f\left(x_{n}\right)+\beta_{n} x_{n}+\gamma_{n} B_{n} x_{n}+\delta_{n} S_{n} G x_{n}, \quad \forall n \geq 0,
$$

where $\left\{\alpha_{n}\right\},\left\{\beta_{n}\right\},\left\{\gamma_{n}\right\}$, and $\left\{\delta_{n}\right\}$ are the sequences in $(0,1)$ such that $\alpha_{n}+\beta_{n}+\gamma_{n}+\delta_{n}=1$ for all $n \geq 0$. Suppose that the following conditions hold:

(i) $\lim _{n \rightarrow \infty} \alpha_{n}=0$ and $\sum_{n=0}^{\infty} \alpha_{n}=\infty$;

(ii) $\left\{\gamma_{n}\right\},\left\{\delta_{n}\right\} \subset[c, d]$ for some $c, d \in(0,1)$;

(iii) $\lim _{n \rightarrow \infty}\left(\left|\beta_{n}-\beta_{n-1}\right|+\left|\gamma_{n}-\gamma_{n-1}\right|+\left|\delta_{n}-\delta_{n-1}\right|\right)=0$;

(iv) $0<\liminf _{n \rightarrow \infty} \beta_{n} \leq \lim \sup _{n \rightarrow \infty} \beta_{n}<1$.

Assume that $\sum_{n=1}^{\infty} \sup _{x \in D}\left\|S_{n} x-S_{n-1} x\right\|<\infty$ for any bounded subset $D$ of $C$ and let $S$ be a mapping of $C$ into itself defined by $S x=\lim _{n \rightarrow \infty} S_{n} x$ for all $x \in C$ and suppose that $\operatorname{Fix}(S)=$ $\bigcap_{i=0}^{\infty} \operatorname{Fix}\left(S_{i}\right)$. Then, there hold the following:

(I) $\lim _{n \rightarrow \infty}\left\|x_{n+1}-x_{n}\right\|=0$;

(II) the sequence $\left\{x_{n}\right\}_{n=0}^{\infty}$ converges strongly to some $q \in$ $F$ which is the unique solution of the variational inequality problem (VIP)

$$
\langle(I-f) q, J(q-p)\rangle \leq 0, \quad \forall p \in F,
$$

provided $\beta_{n} \equiv \beta$ for some fixed $\beta \in(0,1)$.

Proof. First of all, since $0<\lambda_{i}<\left[\widehat{\alpha}_{i} / \kappa^{2}\right]$ for $i=0,1, \ldots$, it is easy to see that $G_{i}$ is a nonexpansive mapping for each $i=$ $0,1, \ldots$. Since $B_{n}: C \rightarrow C$ is the $W$-mapping generated by $G_{n}, G_{n-1}, \ldots, G_{0}$, and $\rho_{n}, \rho_{n-1}, \ldots, \rho_{0}$, by Lemma 16 we know that, for each $x \in C$ and $k \geq 0$, the limit $\lim _{n \rightarrow \infty} U_{n, k} x$ exists. Moreover, one can define a mapping $B: C \rightarrow C$ as follows:

$$
B x=\lim _{n \rightarrow \infty} B_{n} x=\lim _{n \rightarrow \infty} U_{n, 0} x
$$

for every $x \in C$. That is, such a $B$ is the $W$-mapping generated by the sequences $\left\{G_{n}\right\}_{n=0}^{\infty}$ and $\left\{\rho_{n}\right\}_{n=0}^{\infty}$. According to Lemma 17, we know that $\operatorname{Fix}(B)=\bigcap_{i=0}^{\infty} \operatorname{Fix}\left(G_{i}\right)$. From Lemma 21 and the definition of $G_{i}$, we have $\operatorname{Fix}\left(G_{i}\right)=\operatorname{VI}\left(C, A_{i}\right)$ for each $i=0,1, \ldots$. Hence, we have

$$
\operatorname{Fix}(B)=\bigcap_{i=0}^{\infty} \operatorname{Fix}\left(G_{i}\right)=\bigcap_{i=0}^{\infty} \operatorname{VI}\left(C, A_{i}\right)
$$

Next, let us show that the sequence $\left\{x_{n}\right\}$ is bounded. Indeed, take a fixed $p \in F$ arbitrarily. Then, we get $p=G p$,
$p=B_{n} p$, and $p=S_{n} p$ for all $n \geq 0$. By Lemma 23, we know that $G$ is nonexpansive. Then, from (178), we have

$$
\begin{aligned}
\| x_{n+1}- & p \| \\
\leq & \alpha_{n}\left\|f\left(x_{n}\right)-p\right\|+\beta_{n}\left\|x_{n}-p\right\| \\
& +\gamma_{n}\left\|B_{n} x_{n}-p\right\|+\delta_{n}\left\|S_{n} G x_{n}-p\right\| \\
\leq & \alpha_{n}\left(\left\|f\left(x_{n}\right)-f(p)\right\|+\|f(p)-p\|\right) \\
& +\beta_{n}\left\|x_{n}-p\right\|+\gamma_{n}\left\|x_{n}-p\right\|+\delta_{n}\left\|G x_{n}-p\right\| \\
\leq & \alpha_{n}\left(\rho\left\|x_{n}-p\right\|+\|f(p)-p\|\right) \\
& +\beta_{n}\left\|x_{n}-p\right\|+\gamma_{n}\left\|x_{n}-p\right\|+\delta_{n}\left\|x_{n}-p\right\| \\
= & \left(1-\alpha_{n}(1-\rho)\right)\left\|x_{n}-p\right\|+\alpha_{n}(1-\rho) \frac{\|f(p)-p\|}{1-\rho} \\
\leq & \max \left\{\left\|x_{n}-p\right\|, \frac{\|f(p)-p\|}{1-\rho}\right\} .
\end{aligned}
$$

By induction, we obtain

$$
\left\|x_{n}-p\right\| \leq \max \left\{\left\|x_{0}-p\right\|, \frac{\|f(p)-p\|}{1-\rho}\right\}, \quad \forall n \geq 0 .
$$

Hence, $\left\{x_{n}\right\}$ is bounded, and so are the sequences $\left\{G x_{n}\right\}$ and $\left\{f\left(x_{n}\right)\right\}$.

Let us show that

$$
\lim _{n \rightarrow \infty}\left\|x_{n+1}-x_{n}\right\|=0
$$

As a matter of fact, observe that $x_{n+1}$ can be rewritten as follows:

$$
x_{n+1}=\beta_{n} x_{n}+\left(1-\beta_{n}\right) z_{n}
$$

where $z_{n}=\left(\alpha_{n} f\left(x_{n}\right)+\gamma_{n} B_{n} x_{n}+\delta_{n} S_{n} G x_{n}\right) /\left(1-\beta_{n}\right)$. Observe that

$$
\begin{aligned}
\left\|z_{n}-z_{n-1}\right\| & \\
= & \| \frac{\alpha_{n} f\left(x_{n}\right)+\gamma_{n} B_{n} x_{n}+\delta_{n} S_{n} G x_{n}}{1-\beta_{n}} \\
& \quad-\frac{\alpha_{n-1} f\left(x_{n-1}\right)+\gamma_{n-1} B_{n-1} x_{n-1}+\delta_{n-1} S_{n-1} G x_{n-1}}{1-\beta_{n-1}} \| \\
= & \left\|\frac{x_{n+1}-\beta_{n} x_{n}}{1-\beta_{n}}-\frac{x_{n}-\beta_{n-1} x_{n-1}}{1-\beta_{n-1}}\right\| \\
= & \| \frac{x_{n+1}-\beta_{n} x_{n}}{1-\beta_{n}}-\frac{x_{n}-\beta_{n-1} x_{n-1}}{1-\beta_{n}} \\
& +\frac{x_{n}-\beta_{n-1} x_{n-1}}{1-\beta_{n}}-\frac{x_{n}-\beta_{n-1} x_{n-1}}{1-\beta_{n-1}} \|
\end{aligned}
$$




$$
\begin{aligned}
& \leq\left\|\frac{x_{n+1}-\beta_{n} x_{n}}{1-\beta_{n}}-\frac{x_{n}-\beta_{n-1} x_{n-1}}{1-\beta_{n}}\right\| \\
& +\left\|\frac{x_{n}-\beta_{n-1} x_{n-1}}{1-\beta_{n}}-\frac{x_{n}-\beta_{n-1} x_{n-1}}{1-\beta_{n-1}}\right\| \\
& =\frac{1}{1-\beta_{n}}\left\|x_{n+1}-\beta_{n} x_{n}-\left(x_{n}-\beta_{n-1} x_{n-1}\right)\right\| \\
& +\left|\frac{1}{1-\beta_{n}}-\frac{1}{1-\beta_{n-1}}\right|\left\|x_{n}-\beta_{n-1} x_{n-1}\right\| \\
& =\frac{1}{1-\beta_{n}}\left\|x_{n+1}-\beta_{n} x_{n}-\left(x_{n}-\beta_{n-1} x_{n-1}\right)\right\| \\
& +\frac{\left|\beta_{n}-\beta_{n-1}\right|}{\left(1-\beta_{n-1}\right)\left(1-\beta_{n}\right)}\left\|x_{n}-\beta_{n-1} x_{n-1}\right\| \\
& =\frac{1}{1-\beta_{n}} \| \alpha_{n} f\left(x_{n}\right)+\gamma_{n} B_{n} x_{n}+\delta_{n} S_{n} G x_{n}-\alpha_{n-1} f\left(x_{n-1}\right) \\
& -\gamma_{n-1} B_{n-1} x_{n-1}-\delta_{n-1} S_{n-1} G x_{n-1} \| \\
& +\frac{\left|\beta_{n}-\beta_{n-1}\right|}{\left(1-\beta_{n-1}\right)\left(1-\beta_{n}\right)}\left\|x_{n}-\beta_{n-1} x_{n-1}\right\| \\
& \leq \frac{1}{1-\beta_{n}}\left[\alpha_{n}\left\|f\left(x_{n}\right)-f\left(x_{n-1}\right)\right\|\right. \\
& +\gamma_{n}\left\|B_{n} x_{n}-B_{n-1} x_{n-1}\right\| \\
& +\delta_{n}\left\|S_{n} G x_{n}-S_{n-1} G x_{n-1}\right\| \\
& +\left|\alpha_{n}-\alpha_{n-1}\right|\left\|f\left(x_{n-1}\right)\right\| \\
& +\left|\gamma_{n}-\gamma_{n-1}\right|\left\|B_{n-1} x_{n-1}\right\| \\
& \left.+\left|\delta_{n}-\delta_{n-1}\right|\left\|S_{n-1} G x_{n-1}\right\|\right] \\
& +\frac{\left|\beta_{n}-\beta_{n-1}\right|}{\left(1-\beta_{n-1}\right)\left(1-\beta_{n}\right)}\left\|x_{n}-\beta_{n-1} x_{n-1}\right\| \text {. }
\end{aligned}
$$

On the other hand, we note that, for all $n \geq 1$,

$$
\begin{aligned}
& \left\|S_{n} G x_{n}-S_{n-1} G x_{n-1}\right\| \\
& \quad \leq\left\|S_{n} G x_{n}-S_{n} G x_{n-1}\right\|+\left\|S_{n} G x_{n-1}-S_{n-1} G x_{n-1}\right\| \\
& \quad \leq\left\|G x_{n}-G x_{n-1}\right\|+\left\|S_{n} G x_{n-1}-S_{n-1} G x_{n-1}\right\| \\
& \quad \leq\left\|x_{n}-x_{n-1}\right\|+\left\|S_{n} G x_{n-1}-S_{n-1} G x_{n-1}\right\| .
\end{aligned}
$$

Furthermore, by (CY), since $G_{i}$ and $U_{n, i}$ are nonexpansive, we deduce that for each $n \geq 1$

$$
\begin{aligned}
& \left\|B_{n} x_{n}-B_{n-1} x_{n-1}\right\| \\
& \quad \leq\left\|B_{n} x_{n}-B_{n} x_{n-1}\right\|+\left\|B_{n} x_{n-1}-B_{n-1} x_{n-1}\right\| \\
& \quad \leq\left\|x_{n}-x_{n-1}\right\|+\left\|B_{n} x_{n-1}-B_{n-1} x_{n-1}\right\| \\
& \quad=\left\|x_{n}-x_{n-1}\right\|+\left\|\lambda_{0} G_{0} U_{n, 1} x_{n-1}-\lambda_{0} G_{0} U_{n-1,1} x_{n-1}\right\|
\end{aligned}
$$

$$
\begin{aligned}
& \leq\left\|x_{n}-x_{n-1}\right\|+\lambda_{0}\left\|U_{n, 1} x_{n-1}-U_{n-1,1} x_{n-1}\right\| \\
& =\left\|x_{n}-x_{n-1}\right\|+\lambda_{0}\left\|\lambda_{1} G_{1} U_{n, 2} x_{n-1}-\lambda_{1} G_{1} U_{n-1,2} x_{n-1}\right\| \\
& \leq\left\|x_{n}-x_{n-1}\right\|+\lambda_{0} \lambda_{1}\left\|U_{n, 2} x_{n-1}-U_{n-1,2} x_{n-1}\right\| \\
& \vdots \\
& \leq\left\|x_{n}-x_{n-1}\right\|+\left(\prod_{i=0}^{n-1} \lambda_{i}\right)\left\|U_{n, n} x_{n-1}-U_{n-1, n} x_{n-1}\right\| \\
& \leq\left\|x_{n}-x_{n-1}\right\|+M \prod_{i=0}^{n-1} \lambda_{i},
\end{aligned}
$$

for some constant $M>0$. Taking into account $0<$ $\liminf _{n \rightarrow \infty} \beta_{n} \leq \limsup _{n \rightarrow \infty} \beta_{n}<1$, we may assume, without loss of generality, that $\left\{\beta_{n}\right\} \subset[\widehat{c}, \widehat{d}]$. Utilizing (186)(188), we have

$$
\begin{aligned}
& \left\|z_{n}-z_{n-1}\right\| \\
& \leq \frac{1}{1-\beta_{n}}\left[\alpha_{n}\left\|f\left(x_{n}\right)-f\left(x_{n-1}\right)\right\|\right. \\
& +\gamma_{n}\left\|B_{n} x_{n}-B_{n-1} x_{n-1}\right\| \\
& +\delta_{n}\left\|S_{n} G x_{n}-S_{n-1} G x_{n-1}\right\| \\
& +\left|\alpha_{n}-\alpha_{n-1}\right|\left\|f\left(x_{n-1}\right)\right\| \\
& +\left|\gamma_{n}-\gamma_{n-1}\right|\left\|B_{n-1} x_{n-1}\right\| \\
& \left.+\left|\delta_{n}-\delta_{n-1}\right|\left\|S_{n-1} G x_{n-1}\right\|\right] \\
& +\frac{\left|\beta_{n}-\beta_{n-1}\right|}{\left(1-\beta_{n-1}\right)\left(1-\beta_{n}\right)}\left\|x_{n}-\beta_{n-1} x_{n-1}\right\| \\
& \leq \frac{1}{1-\beta_{n}}\left\{\alpha_{n} \rho\left\|x_{n}-x_{n-1}\right\|+\gamma_{n}\left[\left\|x_{n}-x_{n-1}\right\|+M \prod_{i=0}^{n-1} \lambda_{i}\right]\right. \\
& +\delta_{n}\left[\left\|x_{n}-x_{n-1}\right\|+\left\|S_{n} G x_{n-1}-S_{n-1} G x_{n-1}\right\|\right] \\
& +\left|\alpha_{n}-\alpha_{n-1}\right|\left\|f\left(x_{n-1}\right)\right\|+\left|\gamma_{n}-\gamma_{n-1}\right|\left\|B_{n-1} x_{n-1}\right\| \\
& \left.+\left|\delta_{n}-\delta_{n-1}\right|\left\|S_{n-1} G x_{n-1}\right\|\right\} \\
& +\frac{\left|\beta_{n}-\beta_{n-1}\right|}{\left(1-\beta_{n-1}\right)\left(1-\beta_{n}\right)}\left\|x_{n}-\beta_{n-1} x_{n-1}\right\| \\
& =\frac{1}{1-\beta_{n}}\left\{\left(1-\beta_{n}-\alpha_{n}(1-\rho)\right)\left\|x_{n}-x_{n-1}\right\|\right. \\
& +\gamma_{n} M \prod_{i=0}^{n-1} \lambda_{i}+\delta_{n}\left\|S_{n} G x_{n-1}-S_{n-1} G x_{n-1}\right\| \\
& +\left|\alpha_{n}-\alpha_{n-1}\right|\left\|f\left(x_{n-1}\right)\right\|
\end{aligned}
$$




$$
\begin{aligned}
& +\left|\gamma_{n}-\gamma_{n-1}\right||| B_{n-1} x_{n-1}|| \\
& \left.+\left|\delta_{n}-\delta_{n-1}\right| \| S_{n-1} G x_{n-1} \mid\right\} \\
& +\frac{\left|\beta_{n}-\beta_{n-1}\right|}{\left(1-\beta_{n-1}\right)\left(1-\beta_{n}\right)}\left\|x_{n}-\beta_{n-1} x_{n-1}\right\| \\
& =\left(1-\frac{\alpha_{n}(1-\rho)}{1-\beta_{n}}\right)\left\|x_{n}-x_{n-1}\right\|+\frac{\gamma_{n} M}{1-\beta_{n}} \prod_{i=0}^{n-1} \lambda_{i} \\
& +\frac{\delta_{n}}{1-\beta_{n}}\left\|S_{n} G x_{n-1}-S_{n-1} G x_{n-1}\right\|+\frac{1}{1-\beta_{n}} \\
& \times\left[\left|\alpha_{n}-\alpha_{n-1}\right|\left\|f\left(x_{n-1}\right)\right\|+\left|\gamma_{n}-\gamma_{n-1}\right|\left\|B_{n-1} x_{n-1}\right\|\right. \\
& \left.+\left|\delta_{n}-\delta_{n-1}\right|\left\|S_{n-1} G x_{n-1}\right\|\right] \\
& +\frac{\left|\beta_{n}-\beta_{n-1}\right|}{\left(1-\beta_{n-1}\right)\left(1-\beta_{n}\right)}\left\|x_{n}-\beta_{n-1} x_{n-1}\right\| \\
& \leq\left\|x_{n}-x_{n-1}\right\|+M \prod_{i=0}^{n-1} \lambda_{i}+\left\|S_{n} G x_{n-1}-S_{n-1} G x_{n-1}\right\| \\
& +\frac{1}{1-\beta_{n}}\left[\left|\alpha_{n}-\alpha_{n-1}\right|\left\|f\left(x_{n-1}\right)\right\|+\left|\gamma_{n}-\gamma_{n-1}\right|\right. \\
& \left.\times\left\|B_{n-1} x_{n-1}\right\|+\left|\delta_{n}-\delta_{n-1}\right|\left\|S_{n-1} G x_{n-1}\right\|\right] \\
& +\frac{\left|\beta_{n}-\beta_{n-1}\right|}{\left(1-\beta_{n-1}\right)\left(1-\beta_{n}\right)} \\
& \times\left\|\alpha_{n-1} f\left(x_{n-1}\right)+\gamma_{n-1} B_{n-1} x_{n-1}+\delta_{n-1} S_{n-1} G x_{n-1}\right\| \\
& \leq\left\|x_{n}-x_{n-1}\right\| \\
& +M_{1}\left[\prod_{i=0}^{n-1} \lambda_{i}+\left|\alpha_{n}-\alpha_{n-1}\right|\right. \\
& \left.+\left|\gamma_{n}-\gamma_{n-1}\right|+\left|\delta_{n}-\delta_{n-1}\right|+\left|\beta_{n}-\beta_{n-1}\right|\right] \\
& +\left\|S_{n} G x_{n-1}-S_{n-1} G x_{n-1}\right\|,
\end{aligned}
$$

where $\sup _{n \geq 0}\left\{\left(1 /(1-\widehat{d})^{2}\right)\left(\left\|f\left(x_{n}\right)\right\|+\left\|B_{n} x_{n}\right\|+\left\|S_{n} G x_{n}\right\|+M\right)\right\} \leq$ $M_{1}$ for some $M_{1}>0$. Thus, from (189), conditions (i), (iii) and the assumption on $\left\{S_{n}\right\}$, it follows that (noting that $0<\lambda_{i} \leq$ $b<1$, for all $i \geq 0$ )

$$
\lim _{n \rightarrow \infty}\left(\left\|z_{n}-z_{n-1}\right\|-\left\|x_{n}-x_{n-1}\right\|\right) \leq 0 .
$$

Since $0<\liminf _{n \rightarrow \infty} \beta_{n} \leq \lim \sup _{n \rightarrow \infty} \beta_{n}<1$, by Lemma 20 we get

$$
\lim _{n \rightarrow \infty}\left\|x_{n}-z_{n}\right\|=0
$$

Consequently,

$$
\lim _{n \rightarrow \infty}\left\|x_{n+1}-x_{n}\right\|=\lim _{n \rightarrow \infty}\left(1-\beta_{n}\right)\left\|z_{n}-x_{n}\right\|=0 .
$$

Next we show that $\left\|x_{n}-G x_{n}\right\| \rightarrow 0$ as $n \rightarrow \infty$.

Indeed, for simplicity, put $q=\Pi_{C}\left(p-\mu_{2} B_{2} p\right), u_{n}=$ $\Pi_{C}\left(x_{n}-\mu_{2} B_{2} x_{n}\right)$ and $v_{n}=\Pi_{C}\left(u_{n}-\mu_{1} B_{1} u_{n}\right)$. Then, $v_{n}=G x_{n}$ for all $n \geq 0$. From Lemma 26 we have

$$
\begin{aligned}
\| u_{n}- & q \|^{2} \\
& =\left\|\Pi_{C}\left(x_{n}-\mu_{2} B_{2} x_{n}\right)-\Pi_{C}\left(p-\mu_{2} B_{2} p\right)\right\|^{2} \\
& \leq\left\|x_{n}-p-\mu_{2}\left(B_{2} x_{n}-B_{2} p\right)\right\|^{2} \\
& \leq\left\|x_{n}-p\right\|^{2}-2 \mu_{2}\left(\widehat{\beta}_{2}-\kappa^{2} \mu_{2}\right)\left\|B_{2} x_{n}-B_{2} p\right\|^{2}, \\
\| v_{n}- & p \|^{2} \\
& =\left\|\Pi_{C}\left(u_{n}-\mu_{1} B_{1} u_{n}\right)-\Pi_{C}\left(q-\mu_{1} B_{1} q\right)\right\|^{2} \\
& \leq\left\|u_{n}-q-\mu_{1}\left(B_{1} u_{n}-B_{1} q\right)\right\|^{2} \\
& \leq\left\|u_{n}-q\right\|^{2}-2 \mu_{1}\left(\widehat{\beta}_{1}-\kappa^{2} \mu_{1}\right)\left\|B_{1} u_{n}-B_{1} q\right\|^{2} .
\end{aligned}
$$

Substituting (193) for (194), we obtain

$$
\begin{aligned}
\left\|v_{n}-p\right\|^{2} \leq & \left\|x_{n}-p\right\|^{2}-2 \mu_{2}\left(\widehat{\beta}_{2}-\kappa^{2} \mu_{2}\right)\left\|B_{2} x_{n}-B_{2} p\right\|^{2} \\
& -2 \mu_{1}\left(\widehat{\beta}_{1}-\kappa^{2} \mu_{1}\right)\left\|B_{1} u_{n}-B_{1} q\right\|^{2} .
\end{aligned}
$$

By Lemma 8, we have from (178) and (195)

$$
\begin{aligned}
\| x_{n+1}- & p \|^{2} \\
=\| & \alpha_{n}\left(f\left(x_{n}\right)-f(p)\right)+\beta_{n}\left(x_{n}-p\right) \\
& +\gamma_{n}\left(B_{n} x_{n}-p\right)+\delta_{n}\left(S_{n} G x_{n}-p\right) \\
& +\alpha_{n}(f(p)-p) \|^{2} \\
\leq & \| \alpha_{n}\left(f\left(x_{n}\right)-f(p)\right)+\beta_{n}\left(x_{n}-p\right) \\
& +\gamma_{n}\left(B_{n} x_{n}-p\right)+\delta_{n}\left(S_{n} G x_{n}-p\right) \|^{2} \\
& +2 \alpha_{n}\left\langle f(p)-p, J\left(x_{n+1}-p\right)\right\rangle \\
\leq & \alpha_{n}\left\|f\left(x_{n}\right)-f(p)\right\|^{2}+\beta_{n}\left\|x_{n}-p\right\|^{2} \\
& +\gamma_{n}\left\|B_{n} x_{n}-p\right\|^{2}+\delta_{n}\left\|S_{n} G x_{n}-p\right\|^{2} \\
& +2 \alpha_{n}\left\langle f(p)-p, J\left(x_{n+1}-p\right)\right\rangle \\
\leq & \alpha_{n} \rho^{2}\left\|x_{n}-p\right\|^{2}+\beta_{n}\left\|x_{n}-p\right\|^{2} \\
& +\gamma_{n}\left\|x_{n}-p\right\|^{2}+\delta_{n}\left\|v_{n}-p\right\|^{2} \\
& +2 \alpha_{n}\left\langle f(p)-p, J\left(x_{n+1}-p\right)\right\rangle
\end{aligned}
$$




$$
\begin{aligned}
& \leq \alpha_{n} \rho\left\|x_{n}-p\right\|^{2}+\beta_{n}\left\|x_{n}-p\right\|^{2}+\gamma_{n}\left\|x_{n}-p\right\|^{2} \\
& +\delta_{n}\left[\left\|x_{n}-p\right\|^{2}-2 \mu_{2}\left(\widehat{\beta}_{2}-\kappa^{2} \mu_{2}\right)\left\|B_{2} x_{n}-B_{2} p\right\|^{2}\right. \\
& \left.\quad-2 \mu_{1}\left(\widehat{\beta}_{1}-\kappa^{2} \mu_{1}\right)\left\|B_{1} u_{n}-B_{1} q\right\|^{2}\right] \\
& +2 \alpha_{n}\left\langle f(p)-p, J\left(x_{n+1}-p\right)\right\rangle \\
& =\left(1-\alpha_{n}(1-\rho)\right)\left\|x_{n}-p\right\|^{2} \\
& -2 \delta_{n}\left[\mu_{2}\left(\widehat{\beta}_{2}-\kappa^{2} \mu_{2}\right)\left\|B_{2} x_{n}-B_{2} p\right\|^{2}\right. \\
& \left.\quad+\mu_{1}\left(\widehat{\beta}_{1}-\kappa^{2} \mu_{1}\right)\left\|B_{1} u_{n}-B_{1} q\right\|^{2}\right] \\
& +2 \alpha_{n}\left\langle f(p)-p, J\left(x_{n+1}-p\right)\right\rangle \\
& \leq\left\|x_{n}-p\right\|^{2}-2 \delta_{n}\left[\mu_{2}\left(\widehat{\beta}_{2}-\kappa^{2} \mu_{2}\right)\left\|B_{2} x_{n}-B_{2} p\right\|^{2}\right. \\
& \left.\quad+\mu_{1}\left(\widehat{\beta}_{1}-\kappa^{2} \mu_{1}\right)\left\|B_{1} u_{n}-B_{1} q\right\|^{2}\right] \\
& +2 \alpha_{n}\|f(p)-p\|\left\|x_{n+1}-p\right\|,
\end{aligned}
$$

which hence implies that

$$
\begin{aligned}
2 \delta_{n}[ & \mu_{2}\left(\widehat{\beta}_{2}-\kappa^{2} \mu_{2}\right)\left\|B_{2} x_{n}-B_{2} p\right\|^{2} \\
+ & \left.\mu_{1}\left(\widehat{\beta}_{1}-\kappa^{2} \mu_{1}\right)\left\|B_{1} u_{n}-B_{1} q\right\|^{2}\right] \\
\leq & \left\|x_{n}-p\right\|^{2}-\left\|x_{n+1}-p\right\|^{2} \\
& +2 \alpha_{n}\|f(p)-p\|\left\|x_{n+1}-p\right\| \\
\leq & \left(\left\|x_{n}-p\right\|+\left\|x_{n+1}-p\right\|\right)\left\|x_{n}-x_{n+1}\right\| \\
& +2 \alpha_{n}\|f(p)-p\|\left\|x_{n+1}-p\right\| .
\end{aligned}
$$

Since $\left\|x_{n}-x_{n+1}\right\| \rightarrow 0,0<\mu_{i}<\widehat{\beta}_{i} / \kappa^{2}$ for $i=1,2$, and $\left\{x_{n}\right\}$ is bounded, we obtain from conditions (i), (ii) that

$$
\lim _{n \rightarrow \infty}\left\|B_{2} x_{n}-B_{2} p\right\|=0, \quad \lim _{n \rightarrow \infty}\left\|B_{1} u_{n}-B_{1} q\right\|=0 .
$$

Utilizing Proposition 6 and Lemma 9, we have

$$
\begin{aligned}
\left\|u_{n}-q\right\|^{2} & \\
= & \left\|\Pi_{C}\left(x_{n}-\mu_{2} B_{2} x_{n}\right)-\Pi_{C}\left(p-\mu_{2} B_{2} p\right)\right\|^{2} \\
\leq & \left\langle x_{n}-\mu_{2} B_{2} x_{n}-\left(p-\mu_{2} B_{2} p\right), J\left(u_{n}-q\right)\right\rangle \\
= & \left\langle x_{n}-p, J\left(u_{n}-q\right)\right\rangle+\mu_{2}\left\langle B_{2} p-B_{2} x_{n}, J\left(u_{n}-q\right)\right\rangle \\
\leq & \frac{1}{2}\left[\left\|x_{n}-p\right\|^{2}+\left\|u_{n}-q\right\|^{2}\right. \\
& \left.\quad-g_{1}\left(\left\|x_{n}-u_{n}-(p-q)\right\|\right)\right] \\
& +\mu_{2}\left\|B_{2} p-B_{2} x_{n}\right\|\left\|u_{n}-q\right\|,
\end{aligned}
$$

which implies that

$$
\begin{aligned}
\left\|u_{n}-q\right\|^{2} \leq & \left\|x_{n}-p\right\|^{2}-g_{1}\left(\left\|x_{n}-u_{n}-(p-q)\right\|\right) \\
& +2 \mu_{2}\left\|B_{2} p-B_{2} x_{n}\right\|\left\|u_{n}-q\right\| .
\end{aligned}
$$

In the same way, we derive

$$
\begin{aligned}
\| v_{n}- & p \|^{2} \\
= & \left\|\Pi_{C}\left(u_{n}-\mu_{1} B_{1} u_{n}\right)-\Pi_{C}\left(q-\mu_{1} B_{1} q\right)\right\|^{2} \\
\leq & \left\langle u_{n}-\mu_{1} B_{1} u_{n}-\left(q-\mu_{1} B_{1} q\right), J\left(v_{n}-p\right)\right\rangle \\
= & \left\langle u_{n}-q, J\left(v_{n}-p\right)\right\rangle+\mu_{1}\left\langle B_{1} q-B_{1} u_{n}, J\left(v_{n}-p\right)\right\rangle \\
\leq & \frac{1}{2}\left[\left\|u_{n}-q\right\|^{2}+\left\|v_{n}-p\right\|^{2}\right. \\
& \left.\quad-g_{2}\left(\left\|u_{n}-v_{n}+(p-q)\right\|\right)\right] \\
& +\mu_{1}\left\|B_{1} q-B_{1} u_{n}\right\|\left\|v_{n}-p\right\|,
\end{aligned}
$$

which implies that

$$
\begin{gathered}
\left\|v_{n}-p\right\|^{2} \leq \\
\quad\left\|u_{n}-q\right\|^{2}-g_{2}\left(\left\|u_{n}-v_{n}+(p-q)\right\|\right) \\
+2 \mu_{1}\left\|B_{1} q-B_{1} u_{n}\right\|\left\|v_{n}-p\right\| .
\end{gathered}
$$

Substituting (200) for (202), we get

$$
\begin{aligned}
&\left\|v_{n}-p\right\|^{2} \\
& \leq\left\|x_{n}-p\right\|^{2}-g_{1}\left(\left\|x_{n}-u_{n}-(p-q)\right\|\right) \\
&-g_{2}\left(\left\|u_{n}-v_{n}+(p-q)\right\|\right) \\
&+2 \mu_{2}\left\|B_{2} p-B_{2} x_{n}\right\|\left\|u_{n}-q\right\| \\
&+2 \mu_{1}\left\|B_{1} q-B_{1} u_{n}\right\|\left\|v_{n}-p\right\| .
\end{aligned}
$$

By Lemma 8, we have from (196) and (203)

$$
\begin{aligned}
& \left\|x_{n+1}-p\right\|^{2} \\
& \quad \leq \alpha_{n} \rho^{2}\left\|x_{n}-p\right\|^{2}+\beta_{n}\left\|x_{n}-p\right\|^{2}+\gamma_{n}\left\|x_{n}-p\right\|^{2} \\
& \quad+\delta_{n}\left\|v_{n}-p\right\|^{2}+2 \alpha_{n}\left\langle f(p)-p, J\left(x_{n+1}-p\right)\right\rangle
\end{aligned}
$$




$$
\begin{aligned}
& \leq \alpha_{n} \rho\left\|x_{n}-p\right\|^{2}+\beta_{n}\left\|x_{n}-p\right\|^{2}+\gamma_{n}\left\|x_{n}-p\right\|^{2} \\
& +\delta_{n}\left[\left\|x_{n}-p\right\|^{2}-g_{1}\left(\left\|x_{n}-u_{n}-(p-q)\right\|\right)\right. \\
& \quad-g_{2}\left(\left\|u_{n}-v_{n}+(p-q)\right\|\right)+2 \mu_{2}\left\|B_{2} p-B_{2} x_{n}\right\| \\
& \left.\quad \times\left\|u_{n}-q\right\|+2 \mu_{1}\left\|B_{1} q-B_{1} u_{n}\right\|\left\|v_{n}-p\right\|\right] \\
& +2 \alpha_{n}\left\langle f(p)-p, J\left(x_{n+1}-p\right)\right\rangle \\
& \leq\left(1-\alpha_{n}(1-\rho)\right)\left\|x_{n}-p\right\|^{2} \\
& -\delta_{n}\left[g_{1}\left(\left\|x_{n}-u_{n}-(p-q)\right\|\right)\right. \\
& \left.\quad+g_{2}\left(\left\|u_{n}-v_{n}+(p-q)\right\|\right)\right] \\
& +2 \mu_{2}\left\|B_{2} p-B_{2} x_{n}\right\|\left\|u_{n}-q\right\|+2 \mu_{1}\left\|B_{1} q-B_{1} u_{n}\right\| \\
& \times\left\|v_{n}-p\right\|+2 \alpha_{n}\|f(p)-p\|\left\|x_{n+1}-p\right\| \\
& \leq\left\|x_{n}-p\right\|^{2}-\delta_{n}\left[g_{1}\left(\left\|x_{n}-u_{n}-(p-q)\right\|\right)\right. \\
& \left.\quad+g_{2}\left(\left\|u_{n}-v_{n}+(p-q)\right\|\right)\right] \\
& +2 \mu_{2}\left\|B_{2} p-B_{2} x_{n}\right\|\left\|u_{n}-q\right\|+2 \mu_{1}\left\|B_{1} q-B_{1} u_{n}\right\| \\
& \times\left\|v_{n}-p\right\|+2 \alpha_{n}\|f(p)-p\|\left\|x_{n+1}-p\right\|
\end{aligned}
$$

which hence leads to

$$
\begin{aligned}
\delta_{n}\left[g_{1}(\right. & \left.\left.\left\|x_{n}-u_{n}-(p-q)\right\|\right)+g_{2}\left(\left\|u_{n}-v_{n}+(p-q)\right\|\right)\right] \\
\leq & \left\|x_{n}-p\right\|^{2}-\left\|x_{n+1}-p\right\|^{2} \\
& +2 \mu_{2}\left\|B_{2} p-B_{2} x_{n}\right\|\left\|u_{n}-q\right\| \\
& +2 \mu_{1}\left\|B_{1} q-B_{1} u_{n}\right\|\left\|v_{n}-p\right\| \\
& +2 \alpha_{n}\|f(p)-p\|\left\|x_{n+1}-p\right\| \\
\leq & \left(\left\|x_{n}-p\right\|+\left\|x_{n+1}-p\right\|\right)\left\|x_{n}-x_{n+1}\right\| \\
& +2 \mu_{2}\left\|B_{2} p-B_{2} x_{n}\right\|\left\|u_{n}-q\right\| \\
& +2 \mu_{1}\left\|B_{1} q-B_{1} u_{n}\right\|\left\|v_{n}-p\right\| \\
& +2 \alpha_{n}\|f(p)-p\|\left\|x_{n+1}-p\right\| .
\end{aligned}
$$

From (198), (205), conditions (i), (ii) and the boundedness of $\left\{x_{n}\right\},\left\{u_{n}\right\}$, and $\left\{v_{n}\right\}$, we deduce that

$$
\begin{aligned}
& \lim _{n \rightarrow \infty} g_{1}\left(\left\|x_{n}-u_{n}-(p-q)\right\|\right)=0, \\
& \lim _{n \rightarrow \infty} g_{2}\left(\left\|u_{n}-v_{n}+(p-q)\right\|\right)=0 .
\end{aligned}
$$

Utilizing the properties of $g_{1}$ and $g_{2}$, we deduce that

$$
\begin{aligned}
& \lim _{n \rightarrow \infty}\left\|x_{n}-u_{n}-(p-q)\right\|=0, \\
& \lim _{n \rightarrow \infty}\left\|u_{n}-v_{n}+(p-q)\right\|=0 .
\end{aligned}
$$

From (207), we get

$$
\begin{aligned}
\left\|x_{n}-v_{n}\right\| \leq & \left\|x_{n}-u_{n}-(p-q)\right\| \\
& +\left\|u_{n}-v_{n}+(p-q)\right\| \longrightarrow 0 \quad \text { as } n \rightarrow \infty .
\end{aligned}
$$

That is,

$$
\lim _{n \rightarrow \infty}\left\|x_{n}-G x_{n}\right\|=0
$$

Next, let us show that

$$
\lim _{n \rightarrow \infty}\left\|S_{n} G x_{n}-x_{n}\right\|=0, \quad \lim _{n \rightarrow \infty}\left\|B_{n} x_{n}-x_{n}\right\|=0 .
$$

Indeed, observe that $x_{n+1}$ can be rewritten as follows:

$$
\begin{aligned}
x_{n+1}= & \alpha_{n} f\left(x_{n}\right)+\beta_{n} x_{n}+\gamma_{n} B_{n} x_{n}+\delta_{n} S_{n} G x_{n} \\
= & \alpha_{n} f\left(x_{n}\right)+\beta_{n} x_{n}+\left(\gamma_{n}+\delta_{n}\right) \\
& \times \frac{\gamma_{n} B_{n} x_{n}+\delta_{n} S_{n} G x_{n}}{\gamma_{n}+\delta_{n}} \\
= & \alpha_{n} f\left(x_{n}\right)+\beta_{n} x_{n}+e_{n} \widehat{z}_{n},
\end{aligned}
$$

where $e_{n}=\gamma_{n}+\delta_{n}$ and $\widehat{z}_{n}=\left(\gamma_{n} B_{n} x_{n}+\delta_{n} S_{n} G x_{n}\right) /\left(\gamma_{n}+\delta_{n}\right)$. Utilizing Lemma 11 and (211), we have

$$
\begin{aligned}
\left\|x_{n+1}-p\right\|^{2} & \left\|\alpha_{n}\left(f\left(x_{n}\right)-p\right)+\beta_{n}\left(x_{n}-p\right)+e_{n}\left(\widehat{z}_{n}-p\right)\right\|^{2} \\
\leq & \alpha_{n}\left\|f\left(x_{n}\right)-p\right\|^{2}+\beta_{n}\left\|x_{n}-p\right\|^{2} \\
& +e_{n}\left\|\widehat{z}_{n}-p\right\|^{2}-\beta_{n} e_{n} g_{3}\left(\left\|\widehat{z}_{n}-x_{n}\right\|\right) \\
= & \alpha_{n}\left\|f\left(x_{n}\right)-p\right\|^{2}+\beta_{n}\left\|x_{n}-p\right\|^{2} \\
& -\beta_{n} e_{n} g_{3}\left(\left\|\widehat{z}_{n}-x_{n}\right\|\right)+e_{n}\left\|\frac{\gamma_{n} B_{n} x_{n}+\delta_{n} S_{n} G x_{n}}{\gamma_{n}+\delta_{n}}-p\right\|^{2} \\
= & \alpha_{n}\left\|f\left(x_{n}\right)-p\right\|^{2}+\beta_{n}\left\|x_{n}-p\right\|^{2}-\beta_{n} e_{n} g_{3}\left(\left\|\widehat{z}_{n}-x_{n}\right\|\right) \\
& +e_{n}\left\|\frac{\gamma_{n}}{\gamma_{n}+\delta_{n}}\left(B_{n} x_{n}-p\right)+\frac{\delta_{n}}{\gamma_{n}+\delta_{n}}\left(S_{n} G x_{n}-p\right)\right\|^{2}
\end{aligned}
$$




$$
\begin{aligned}
\leq & \alpha_{n}\left\|f\left(x_{n}\right)-p\right\|^{2}+\beta_{n}\left\|x_{n}-p\right\|^{2}-\beta_{n} e_{n} g_{3}\left(\left\|\widehat{z}_{n}-x_{n}\right\|\right) \\
& +e_{n}\left[\frac{\gamma_{n}}{\gamma_{n}+\delta_{n}}\left\|B_{n} x_{n}-p\right\|^{2}+\frac{\delta_{n}}{\gamma_{n}+\delta_{n}}\left\|S_{n} G x_{n}-p\right\|^{2}\right] \\
\leq & \alpha_{n}\left\|f\left(x_{n}\right)-p\right\|^{2}+\beta_{n}\left\|x_{n}-p\right\|^{2}-\beta_{n} e_{n} g_{3}\left(\left\|\bar{z}_{n}-x_{n}\right\|\right) \\
& +e_{n}\left[\frac{\gamma_{n}}{\gamma_{n}+\delta_{n}}\left\|x_{n}-p\right\|+\frac{\delta_{n}}{\gamma_{n}+\delta_{n}}\left\|G x_{n}-p\right\|^{2}\right] \\
\leq & \alpha_{n}\left\|f\left(x_{n}\right)-p\right\|^{2}+\beta_{n}\left\|x_{n}-p\right\|^{2}-\beta_{n} e_{n} g_{3}\left(\left\|\bar{z}_{n}-x_{n}\right\|\right) \\
& +e_{n}\left[\frac{\gamma_{n}}{\gamma_{n}+\delta_{n}}\left\|x_{n}-p\right\|+\frac{\delta_{n}}{\gamma_{n}+\delta_{n}}\left\|x_{n}-p\right\|^{2}\right] \\
= & \alpha_{n}\left\|f\left(x_{n}\right)-p\right\|^{2}+\left(1-\alpha_{n}\right)\left\|x_{n}-p\right\|^{2} \\
& -\beta_{n} e_{n} g_{3}\left(\left\|\widehat{z}_{n}-x_{n}\right\|\right) \\
\leq & \alpha_{n}\left\|f\left(x_{n}\right)-p\right\|^{2}+\left\|x_{n}-p\right\|^{2}-\beta_{n} e_{n} g_{3}\left(\left\|\widehat{z}_{n}-x_{n}\right\|\right),
\end{aligned}
$$

which hence implies that

$$
\begin{aligned}
& \beta_{n} e_{n} g_{3}\left(\left\|\bar{z}_{n}-x_{n}\right\|\right) \\
& \leq \alpha_{n}\left\|f\left(x_{n}\right)-p\right\|^{2}+\left\|x_{n}-p\right\|^{2}-\left\|x_{n+1}-p\right\|^{2} \\
& \leq \alpha_{n}\left\|f\left(x_{n}\right)-p\right\|^{2}+\left(\left\|x_{n}-p\right\|+\left\|x_{n+1}-p\right\|\right) \\
& \quad \times\left\|x_{n}-x_{n+1}\right\| .
\end{aligned}
$$

Utilizing (184), conditions (i), (ii), (iv), and the boundedness of $\left\{x_{n}\right\}$ and $\left\{f\left(x_{n}\right)\right\}$, we get

$$
\lim _{n \rightarrow \infty} g_{3}\left(\left\|\widehat{z}_{n}-x_{n}\right\|\right)=0 .
$$

From the properties of $g_{3}$, we have

$$
\lim _{n \rightarrow \infty}\left\|\widehat{z}_{n}-x_{n}\right\|=0
$$

Utilizing Lemma 15 and the definition of $\widehat{z}_{n}$, we have

$$
\begin{aligned}
\| \bar{z}_{n}- & p \|^{2} \\
= & \left\|\frac{\gamma_{n} B_{n} x_{n}+\delta_{n} S_{n} G x_{n}}{\gamma_{n}+\delta_{n}}-p\right\|^{2} \\
= & \left\|\frac{\gamma_{n}}{\gamma_{n}+\delta_{n}}\left(B_{n} x_{n}-p\right)+\frac{\delta_{n}}{\gamma_{n}+\delta_{n}}\left(S_{n} G x_{n}-p\right)\right\|^{2} \\
\leq & \frac{\gamma_{n}}{\gamma_{n}+\delta_{n}}\left\|B_{n} x_{n}-p\right\|^{2}+\frac{\delta_{n}}{\gamma_{n}+\delta_{n}}\left\|S_{n} G x_{n}-p\right\|^{2} \\
& -\frac{\gamma_{n} \delta_{n}}{\left(\gamma_{n}+\delta_{n}\right)^{2}} g_{4}\left(\left\|S_{n} G x_{n}-B_{n} x_{n}\right\|\right)
\end{aligned}
$$

$$
\begin{aligned}
\leq & \frac{\gamma_{n}}{\gamma_{n}+\delta_{n}}\left\|x_{n}-p\right\|^{2}+\frac{\delta_{n}}{\gamma_{n}+\delta_{n}}\left\|G x_{n}-p\right\|^{2} \\
& -\frac{\gamma_{n} \delta_{n}}{\left(\gamma_{n}+\delta_{n}\right)^{2}} g_{4}\left(\left\|S_{n} G x_{n}-B_{n} x_{n}\right\|\right) \\
\leq & \frac{\gamma_{n}}{\gamma_{n}+\delta_{n}}\left\|x_{n}-p\right\|^{2}+\frac{\delta_{n}}{\gamma_{n}+\delta_{n}}\left\|x_{n}-p\right\|^{2} \\
& -\frac{\gamma_{n} \delta_{n}}{\left(\gamma_{n}+\delta_{n}\right)^{2}} g_{4}\left(\left\|S_{n} G x_{n}-B_{n} x_{n}\right\|\right) \\
= & \left\|x_{n}-p\right\|^{2}-\frac{\gamma_{n} \delta_{n}}{\left(\gamma_{n}+\delta_{n}\right)^{2}} g_{4}\left(\left\|S_{n} G x_{n}-B_{n} x_{n}\right\|\right),
\end{aligned}
$$

which hence yields

$$
\begin{aligned}
& \frac{\gamma_{n} \delta_{n}}{\left(\gamma_{n}+\delta_{n}\right)^{2}} g_{4}\left(\left\|S_{n} G x_{n}-B_{n} x_{n}\right\|\right) \\
& \quad \leq\left\|x_{n}-p\right\|^{2}-\left\|\widehat{z}_{n}-p\right\|^{2} \\
& \quad \leq\left(\left\|x_{n}-p\right\|+\left\|\widehat{z}_{n}-p\right\|\right)\left\|x_{n}-\widehat{z}_{n}\right\| .
\end{aligned}
$$

Since $\left\{x_{n}\right\}$ and $\left\{\widehat{z}_{n}\right\}$ are bounded and $\left\|\widehat{z}_{n}-x_{n}\right\| \rightarrow 0$ as $n \rightarrow$ $\infty$, we deduce from condition (ii) that

$$
\lim _{n \rightarrow \infty} g_{4}\left(\left\|S_{n} G x_{n}-B_{n} x_{n}\right\|\right)=0 .
$$

From the properties of $g_{4}$, we have

$$
\lim _{n \rightarrow \infty}\left\|S_{n} G x_{n}-B_{n} x_{n}\right\|=0
$$

On the other hand, $x_{n+1}$ can also be rewritten as follows:

$$
\begin{aligned}
x_{n+1} & =\alpha_{n} f\left(x_{n}\right)+\beta_{n} x_{n}+\gamma_{n} B_{n} x_{n}+\delta_{n} S_{n} G x_{n} \\
& =\beta_{n} x_{n}+\gamma_{n} B_{n} x_{n}+\left(\alpha_{n}+\delta_{n}\right) \frac{\alpha_{n} f\left(x_{n}\right)+\delta_{n} S_{n} G x_{n}}{\alpha_{n}+\delta_{n}} \\
& =\beta_{n} x_{n}+\gamma_{n} B_{n} x_{n}+d_{n} \widetilde{z}_{n},
\end{aligned}
$$

where $d_{n}=\alpha_{n}+\delta_{n}$ and $\widetilde{z}_{n}=\left(\alpha_{n} f\left(x_{n}\right)+\delta_{n} S_{n} G x_{n}\right) /\left(\alpha_{n}+\delta_{n}\right)$. Utilizing Lemma 11 and the convexity of $\|\cdot\|^{2}$, we have

$$
\begin{aligned}
& \left\|x_{n+1}-p\right\|^{2} \\
& =\left\|\beta_{n}\left(x_{n}-p\right)+\gamma_{n}\left(B_{n} x_{n}-p\right)+d_{n}\left(\widetilde{z}_{n}-p\right)\right\|^{2} \\
& \leq \beta_{n}\left\|x_{n}-p\right\|^{2}+\gamma_{n}\left\|B_{n} x_{n}-p\right\|^{2} \\
& \quad+d_{n}\left\|\widetilde{z}_{n}-p\right\|^{2}-\beta_{n} \gamma_{n} g_{5}\left(\left\|x_{n}-B_{n} x_{n}\right\|\right)
\end{aligned}
$$




$$
\begin{aligned}
& =\beta_{n}\left\|x_{n}-p\right\|^{2}+\gamma_{n}\left\|B_{n} x_{n}-p\right\|^{2} \\
& +d_{n}\left\|\frac{\alpha_{n} f\left(x_{n}\right)+\delta_{n} S_{n} G x_{n}}{\alpha_{n}+\delta_{n}}-p\right\|^{2} \\
& -\beta_{n} \gamma_{n} g_{5}\left(\left\|x_{n}-B_{n} x_{n}\right\|\right) \\
& =\beta_{n}\left\|x_{n}-p\right\|^{2}+\gamma_{n}\left\|B_{n} x_{n}-p\right\|^{2} \\
& +d_{n}\left\|\frac{\alpha_{n}}{\alpha_{n}+\delta_{n}}\left(f\left(x_{n}\right)-p\right)+\frac{\delta_{n}}{\alpha_{n}+\delta_{n}}\left(S_{n} G x_{n}-p\right)\right\|^{2} \\
& -\beta_{n} \gamma_{n} g_{5}\left(\left\|x_{n}-B_{n} x_{n}\right\|\right) \\
& \leq \beta_{n}\left\|x_{n}-p\right\|^{2}+\gamma_{n}\left\|x_{n}-p\right\|^{2} \\
& +d_{n}\left[\frac{\alpha_{n}}{\alpha_{n}+\delta_{n}}\left\|f\left(x_{n}\right)-p\right\|^{2}+\frac{\delta_{n}}{\alpha_{n}+\delta_{n}}\left\|S_{n} G x_{n}-p\right\|^{2}\right] \\
& -\beta_{n} \gamma_{n} g_{5}\left(\left\|x_{n}-B_{n} x_{n}\right\|\right) \\
& \leq \beta_{n}\left\|x_{n}-p\right\|^{2}+\gamma_{n}\left\|x_{n}-p\right\|^{2} \\
& +d_{n}\left[\frac{\alpha_{n}}{\alpha_{n}+\delta_{n}}\left\|f\left(x_{n}\right)-p\right\|^{2}+\frac{\delta_{n}}{\alpha_{n}+\delta_{n}}\left\|G x_{n}-p\right\|^{2}\right] \\
& -\beta_{n} \gamma_{n} g_{5}\left(\left\|x_{n}-B_{n} x_{n}\right\|\right) \\
& \leq \beta_{n}\left\|x_{n}-p\right\|^{2}+\gamma_{n}\left\|x_{n}-p\right\|^{2} \\
& +d_{n}\left[\frac{\alpha_{n}}{\alpha_{n}+\delta_{n}}\left\|f\left(x_{n}\right)-p\right\|^{2}+\frac{\delta_{n}}{\alpha_{n}+\delta_{n}}\left\|x_{n}-p\right\|^{2}\right] \\
& -\beta_{n} \gamma_{n} g_{5}\left(\left\|x_{n}-B_{n} x_{n}\right\|\right) \\
& =\alpha_{n}\left\|f\left(x_{n}\right)-p\right\|^{2}+\left(1-\alpha_{n}\right)\left\|x_{n}-p\right\|^{2} \\
& -\beta_{n} \gamma_{n} g_{5}\left(\left\|x_{n}-B_{n} x_{n}\right\|\right) \\
& \leq \alpha_{n}\left\|f\left(x_{n}\right)-p\right\|^{2}+\left\|x_{n}-p\right\|^{2}-\beta_{n} \gamma_{n} g_{5}\left(\left\|x_{n}-B_{n} x_{n}\right\|\right) \text {, }
\end{aligned}
$$

which hence implies that

$$
\begin{aligned}
\beta_{n} \gamma_{n} g_{5} & \left(\left\|x_{n}-B_{n} x_{n}\right\|\right) \\
\leq & \alpha_{n}\left\|f\left(x_{n}\right)-p\right\|^{2}+\left\|x_{n}-p\right\|^{2}-\left\|x_{n+1}-p\right\|^{2} \\
\leq & \alpha_{n}\left\|f\left(x_{n}\right)-p\right\|^{2}+\left(\left\|x_{n}-p\right\|+\left\|x_{n+1}-p\right\|\right) \\
& \times\left\|x_{n}-x_{n+1}\right\| .
\end{aligned}
$$

From (184), conditions (i), (ii), (iv), and the boundedness of $\left\{x_{n}\right\}$ and $\left\{f\left(x_{n}\right)\right\}$, we have

$$
\lim _{n \rightarrow \infty} g_{5}\left(\left\|x_{n}-B_{n} x_{n}\right\|\right)=0 .
$$

Utilizing the properties of $g_{5}$, we have

$$
\lim _{n \rightarrow \infty}\left\|x_{n}-B_{n} x_{n}\right\|=0
$$

which, together with (219), implies that

$$
\begin{aligned}
\left\|S_{n} G x_{n}-x_{n}\right\| \leq\left\|S_{n} G x_{n}-B_{n} x_{n}\right\|+\left\|B_{n} x_{n}-x_{n}\right\| & \longrightarrow 0 \\
\text { as } n & \rightarrow \infty .
\end{aligned}
$$

That is,

$$
\lim _{n \rightarrow \infty}\left\|S_{n} G x_{n}-x_{n}\right\|=0 \text {. }
$$

We note that

$$
\begin{aligned}
\| x_{n}- & S x_{n} \| \\
& \leq\left\|x_{n}-S_{n} G x_{n}\right\|+\left\|S_{n} G x_{n}-S_{n} x_{n}\right\|+\left\|S_{n} x_{n}-S x_{n}\right\| \\
& \leq\left\|x_{n}-S_{n} G x_{n}\right\|+\left\|G x_{n}-x_{n}\right\|+\left\|S_{n} x_{n}-S x_{n}\right\| .
\end{aligned}
$$

So, in terms of (209), (226), and Lemma 12, we have

$$
\lim _{n \rightarrow \infty}\left\|x_{n}-S x_{n}\right\|=0
$$

Suppose that $\beta_{n} \equiv \beta$ for some fixed $\beta \in(0,1)$ such that $\alpha_{n}+$ $\beta+\gamma_{n}+\delta_{n}=1$ for all $n \geq 0$. Define a mapping $V x=\left(1-\theta_{1}-\right.$ $\left.\theta_{2}\right) S x+\theta_{1} B x+\theta_{2} G x$, where $\theta_{1}, \theta_{2} \in(0,1)$ are two constants with $\theta_{1}+\theta_{2}<1$. Then by Lemmas 14 and 17, we have that $\operatorname{Fix}(V)=\operatorname{Fix}(S) \cap \operatorname{Fix}(B) \cap \operatorname{Fix}(G)=F$. For each $k \geq 1$, let $\left\{p_{k}\right\}$ be a unique element of $C$ such that

$$
p_{k}=\frac{1}{k} f\left(p_{k}\right)+\left(1-\frac{1}{k}\right) V p_{k}
$$

From Lemma 13, we conclude that $p_{k} \rightarrow q \in \operatorname{Fix}(V)=F$ as $k \rightarrow \infty$. Observe that for every $n, k$

$$
\begin{aligned}
\| x_{n+1} & -B p_{k} \| \\
= & \| \alpha_{n}\left(f\left(x_{n}\right)-B p_{k}\right)+\beta\left(x_{n}-B p_{k}\right) \\
& +\gamma_{n}\left(B_{n} x_{n}-B p_{k}\right)+\delta_{n}\left(S_{n} G x_{n}-B p_{k}\right) \| \\
\leq & \alpha_{n}\left\|f\left(x_{n}\right)-B p_{k}\right\|+\beta\left\|x_{n}-B p_{k}\right\|+\gamma_{n}\left\|B_{n} x_{n}-B p_{k}\right\| \\
& +\delta_{n}\left(\left\|S_{n} G x_{n}-B_{n} x_{n}\right\|+\left\|B_{n} x_{n}-B p_{k}\right\|\right) \\
= & \alpha_{n}\left\|f\left(x_{n}\right)-B p_{k}\right\|+\beta\left\|x_{n}-B p_{k}\right\| \\
& +\left(\gamma_{n}+\delta_{n}\right)\left\|B x_{n}-B p_{k}\right\|+\delta_{n}\left\|S_{n} G x_{n}-B_{n} x_{n}\right\| \\
= & \alpha_{n}\left\|f\left(x_{n}\right)-B p_{k}\right\|+\beta\left\|x_{n}-B p_{k}\right\| \\
& +\left(1-\alpha_{n}-\beta\right)\left\|B_{n} x_{n}-B p_{k}\right\|+\delta_{n}\left\|S_{n} G x_{n}-B_{n} x_{n}\right\|
\end{aligned}
$$




$$
\begin{aligned}
\leq & \alpha_{n}\left\|f\left(x_{n}\right)-B p_{k}\right\|+\beta\left\|x_{n}-B p_{k}\right\|+\left(1-\alpha_{n}-\beta\right) \\
& \times\left[\left\|B_{n} x_{n}-B_{n} p_{k}\right\|+\left\|B_{n} p_{k}-B p_{k}\right\|\right] \\
& +\delta_{n}\left\|S_{n} G x_{n}-B_{n} x_{n}\right\| \\
\leq & \alpha_{n}\left\|f\left(x_{n}\right)-B p_{k}\right\|+\beta\left\|x_{n}-B p_{k}\right\| \\
& +\left(1-\alpha_{n}-\beta\right)\left[\left\|x_{n}-p_{k}\right\|+\left\|B_{n} p_{k}-B p_{k}\right\|\right] \\
& +\delta_{n}\left\|S_{n} G x_{n}-B_{n} x_{n}\right\| \\
\leq & \alpha_{n}\left\|f\left(x_{n}\right)-B p_{k}\right\|+\beta\left\|x_{n}-B p_{k}\right\| \\
& +(1-\beta)\left[\left\|x_{n}-p_{k}\right\|+\left\|B_{n} p_{k}-B p_{k}\right\|\right] \\
& +\delta_{n}\left\|S_{n} G x_{n}-B_{n} x_{n}\right\| \\
= & \theta_{n}+\beta\left\|x_{n}-B p_{k}\right\|+(1-\beta)\left\|x_{n}-p_{k}\right\|,
\end{aligned}
$$

where $\theta_{n}=\alpha_{n}\left\|f\left(x_{n}\right)-B p_{k}\right\|+(1-\beta)\left\|B_{n} p_{k}-B p_{k}\right\|+$ $\delta_{n}\left\|S_{n} G x_{n}-B_{n} x_{n}\right\|$. Since $\lim _{n \rightarrow \infty} \alpha_{n}=\lim _{n \rightarrow \infty}\left\|B_{n} p_{k}-B p_{k}\right\|=$ $\lim _{n \rightarrow \infty}\left\|S_{n} G x_{n}-B_{n} x_{n}\right\|=0$, we know that $\theta_{n} \rightarrow 0$ as $n \rightarrow \infty$.

From (230), we obtain

$$
\begin{aligned}
\| x_{n+1}- & B p_{k} \|^{2} \\
\leq & \left(\beta\left\|x_{n}-B p_{k}\right\|+(1-\beta)\left\|x_{n}-p_{k}\right\|\right)^{2} \\
& +\theta_{n}\left[2\left(\beta\left\|x_{n}-B p_{k}\right\|+(1-\beta)\left\|x_{n}-p_{k}\right\|\right)+\theta_{n}\right] \\
= & \beta^{2}\left\|x_{n}-B p_{k}\right\|^{2}+(1-\beta)^{2}\left\|x_{n}-p_{k}\right\|^{2} \\
& +2 \beta(1-\beta)\left\|x_{n}-B p_{k}\right\|\left\|x_{n}-p_{k}\right\|+\tau_{n} \\
\leq & \beta^{2}\left\|x_{n}-B p_{k}\right\|^{2}+(1-\beta)^{2}\left\|x_{n}-p_{k}\right\|^{2} \\
& +\beta(1-\beta)\left(\left\|x_{n}-B p_{k}\right\|^{2}+\left\|x_{n}-p_{k}\right\|^{2}\right)+\tau_{n} \\
= & \beta\left\|x_{n}-B p_{k}\right\|^{2}+(1-\beta)\left\|x_{n}-p_{k}\right\|^{2}+\tau_{n},
\end{aligned}
$$

where $\tau_{n}=\theta_{n}\left[2\left(\beta\left\|x_{n}-B p_{k}\right\|+(1-\beta)\left\|x_{n}-p_{k}\right\|\right)+\theta_{n}\right] \rightarrow 0$ as $n \rightarrow \infty$.

For any Banach limit $\mu$, from (231) we derive

$$
\mu_{n}\left\|x_{n}-B p_{k}\right\|^{2}=\mu_{n}\left\|x_{n+1}-B p_{k}\right\|^{2} \leq \mu_{n}\left\|x_{n}-p_{k}\right\|^{2} .
$$

In addition, note that

$$
\begin{aligned}
&\left\|x_{n}-G p_{k}\right\|^{2} \\
& \leq\left\|x_{n}-G x_{n}+G x_{n}-G p_{k}\right\|^{2} \\
& \leq\left(\left\|x_{n}-G x_{n}\right\|+\left\|x_{n}-p_{k}\right\|\right)^{2} \\
&=\left\|x_{n}-p_{k}\right\|^{2}+\left\|x_{n}-G x_{n}\right\| \\
& \times\left(2\left\|x_{n}-p_{k}\right\|+\left\|x_{n}-G x_{n}\right\|\right),
\end{aligned}
$$

$$
\begin{aligned}
\| x_{n}- & S p_{k} \|^{2} \\
\leq & \left\|x_{n}-S x_{n}+S x_{n}-S p_{k}\right\|^{2} \\
\leq & \left(\left\|x_{n}-S x_{n}\right\|+\left\|x_{n}-p_{k}\right\|\right)^{2} \\
= & \left\|x_{n}-p_{k}\right\|^{2}+\left\|x_{n}-S x_{n}\right\| \\
& \times\left(2\left\|x_{n}-p_{k}\right\|+\left\|x_{n}-S x_{n}\right\|\right) .
\end{aligned}
$$

It is easy to see from (209) and (228) that

$$
\begin{gathered}
\mu_{n}\left\|x_{n}-G p_{k}\right\|^{2} \leq \mu_{n}\left\|x_{n}-p_{k}\right\|^{2}, \\
\mu_{n}\left\|x_{n}-S p_{k}\right\|^{2} \leq \mu_{n}\left\|x_{n}-p_{k}\right\|^{2} .
\end{gathered}
$$

Utilizing (232) and (234), we deduce that

$$
\begin{aligned}
\mu_{n}\left\|x_{n}-V p_{k}\right\|^{2} & \\
= & \mu_{n} \|\left(1-\theta_{1}-\theta_{2}\right)\left(x_{n}-S p_{k}\right) \\
& \quad+\theta_{1}\left(x_{n}-B p_{k}\right)+\theta_{2}\left(x_{n}-G p_{k}\right) \|^{2} \\
\leq & \left(1-\theta_{1}-\theta_{2}\right) \mu_{n}\left\|x_{n}-S p_{k}\right\|^{2} \\
& +\theta_{1} \mu_{n}\left\|x_{n}-B p_{k}\right\|^{2}+\theta_{2} \mu_{n}\left\|x_{n}-G p_{k}\right\|^{2} \\
\leq & \mu_{n}\left\|x_{n}-p_{k}\right\|^{2} .
\end{aligned}
$$

Also, observe that

$$
x_{n}-p_{k}=\frac{1}{k}\left(x_{n}-f\left(p_{k}\right)\right)+\left(1-\frac{1}{k}\right)\left(x_{n}-V p_{k}\right) ;
$$

that is,

$$
\left(1-\frac{1}{k}\right)\left(x_{n}-V p_{k}\right)=x_{n}-p_{k}-\frac{1}{k}\left(x_{n}-f\left(p_{k}\right)\right) .
$$

It follows from Lemma 8(ii) and (237) that

$$
\begin{aligned}
\left(1-\frac{1}{k}\right)^{2}\left\|x_{n}-V p_{k}\right\|^{2} \\
\quad \geq\left\|x_{n}-p_{k}\right\|^{2}-\frac{2}{k}\left\langle x_{n}-p_{k}+p_{k}-f\left(p_{k}\right), J\left(x_{n}-p_{k}\right)\right\rangle \\
\quad=\left(1-\frac{2}{k}\right)\left\|x_{n}-p_{k}\right\|^{2}+\frac{2}{k}\left\langle f\left(p_{k}\right)-p_{k}, J\left(x_{n}-p_{k}\right)\right\rangle .
\end{aligned}
$$

So by (235) and (238), we have

$$
\begin{aligned}
\left(1-\frac{1}{k}\right)^{2} \mu_{n}\left\|x_{n}-p_{k}\right\|^{2} & \\
\geq & \left(1-\frac{2}{k}\right) \mu_{n}\left\|x_{n}-p_{k}\right\|^{2} \\
& +\frac{2}{k} \mu_{n}\left\langle f\left(p_{k}\right)-p_{k}, J\left(x_{n}-p_{k}\right)\right\rangle,
\end{aligned}
$$


and hence

$$
\frac{1}{k^{2}} \mu_{n}\left\|x_{n}-p_{k}\right\|^{2} \geq \frac{2}{k} \mu_{n}\left\langle f\left(p_{k}\right)-p_{k}, J\left(x_{n}-p_{k}\right)\right\rangle
$$

This implies that

$$
\frac{1}{2 k} \mu_{n}\left\|x_{n}-p_{k}\right\|^{2} \geq \mu_{n}\left\langle f\left(p_{k}\right)-p_{k}, J\left(x_{n}-p_{k}\right)\right\rangle .
$$

Since $p_{k} \rightarrow q \in \operatorname{Fix}(V)=F$ as $k \rightarrow \infty$, by the uniform Frechet differentiability of the norm of $X$, we have

$$
\mu_{n}\left\langle f(q)-q, J\left(x_{n}-q\right)\right\rangle \leq 0 .
$$

On the other hand, from (184) and the norm-to-norm uniform continuity of $J$ on bounded subsets of $X$, it follows that

$$
\lim _{n \rightarrow \infty}\left|\left\langle f(q)-q, J\left(x_{n+1}-q\right)\right\rangle-\left\langle f(q)-q, J\left(x_{n}-q\right)\right\rangle\right|=0 .
$$

So, utilizing Lemma 18 we deduce from (242) and (243) that

$$
\limsup _{n \rightarrow \infty}\left\langle f(q)-q, J\left(x_{n}-q\right)\right\rangle \leq 0
$$

which, together with (184) and the norm-to-norm uniform continuity of $J$ on bounded subsets of $X$, implies that

$$
\limsup _{n \rightarrow \infty}\left\langle f(q)-q, J\left(x_{n+1}-q\right)\right\rangle \leq 0 .
$$

Finally, let us show that $x_{n} \rightarrow q$ as $n \rightarrow \infty$. Utilizing Lemma 8 (i), from (178) and the convexity of $\|\cdot\|^{2}$, we get

$$
\begin{aligned}
\left\|x_{n+1}-q\right\|^{2} & \\
= & \| \alpha_{n}\left(f\left(x_{n}\right)-f(q)\right)+\beta_{n}\left(x_{n}-q\right)+\gamma_{n}\left(B_{n} x_{n}-q\right) \\
& +\delta_{n}\left(S_{n} G x_{n}-q\right)+\alpha_{n}(f(q)-q) \|^{2} \\
\leq & \| \alpha_{n}\left(f\left(x_{n}\right)-f(q)\right)+\beta_{n}\left(x_{n}-q\right) \\
& +\gamma_{n}\left(B_{n} x_{n}-q\right)+\delta_{n}\left(S_{n} G x_{n}-q\right) \|^{2} \\
& +2 \alpha_{n}\left\langle f(q)-q, J\left(x_{n+1}-q\right)\right\rangle \\
\leq & \alpha_{n}\left\|f\left(x_{n}\right)-f(q)\right\|^{2}+\beta_{n}\left\|x_{n}-q\right\|^{2} \\
& +\gamma_{n}\left\|B_{n} x_{n}-q\right\|^{2}+\delta_{n}\left\|S_{n} G x_{n}-q\right\|^{2} \\
& +2 \alpha_{n}\left\langle f(q)-q, J\left(x_{n+1}-q\right)\right\rangle
\end{aligned}
$$

$$
\begin{aligned}
\leq & \alpha_{n}\left\|f\left(x_{n}\right)-f(q)\right\|^{2}+\beta_{n}\left\|x_{n}-q\right\|^{2} \\
& +\gamma_{n}\left\|x_{n}-q\right\|^{2}+\delta_{n}\left\|G x_{n}-q\right\|^{2} \\
& +2 \alpha_{n}\left\langle f(q)-q, J\left(x_{n+1}-q\right)\right\rangle \\
\leq & \alpha_{n} \rho\left\|x_{n}-q\right\|^{2}+\beta_{n}\left\|x_{n}-q\right\|^{2}+\gamma_{n}\left\|x_{n}-q\right\|^{2} \\
& +\delta_{n}\left\|x_{n}-q\right\|^{2}+2 \alpha_{n}\left\langle f(q)-q, J\left(x_{n+1}-q\right)\right\rangle \\
= & \left(1-\alpha_{n}(1-\rho)\right)\left\|x_{n}-q\right\|^{2} \\
& +2 \alpha_{n}\left\langle f(q)-q, J\left(x_{n+1}-q\right)\right\rangle \\
= & \left(1-\alpha_{n}(1-\rho)\right)\left\|x_{n}-q\right\|^{2} \\
& +\alpha_{n}(1-\rho) \frac{2\left\langle f(q)-q, J\left(x_{n+1}-q\right)\right\rangle}{1-\rho} .
\end{aligned}
$$

Applying Lemma 7 to (246), we obtain that $x_{n} \rightarrow q$ as $n \rightarrow$ $\infty$. This completes the proof.

Corollary 32. Let $C$ be a nonempty closed convex subset of a uniformly convex and 2-uniformly smooth Banach space X. Let $\Pi_{C}$ be a sunny nonexpansive retraction from $X$ onto $C$. Let $\left\{\rho_{n}\right\}_{n=0}^{\infty}$ be a sequence of positive numbers in $(0, b]$ for some $b \in(0,1)$ and $A_{i}: C \rightarrow X$ an $\widehat{\alpha}_{i}$-inverse strongly accretive mapping for each $i=0,1, \ldots$. Define a mapping $G_{i}: C \rightarrow C$ by $\Pi_{C}\left(I-\lambda_{i} A_{i}\right) x=G_{i} x$ for all $x \in C$ and $i=0,1, \ldots$, where $\lambda_{i} \in\left(0, \widehat{\alpha}_{i} / \kappa^{2}\right]$ and $\kappa$ is the 2 -uniformly smooth constant of $X$. Let $B_{n}: C \rightarrow C$ be the $W$-mapping generated by $G_{n}, G_{n-1}, \ldots, G_{0}$, and $\rho_{n}, \rho_{n-1}, \ldots, \rho_{0}$. Let $V: C \rightarrow C$ be an $\alpha$-strictly pseudocontractive mapping. Let $f: C \rightarrow C$ be a contraction with coefficient $\rho \in(0,1)$. Let $\left\{S_{i}\right\}_{i=0}^{\infty}$ be a countable family of nonexpansive mappings of $C$ into itself such that $F=\left(\bigcap_{i=0}^{\infty} \operatorname{Fix}\left(S_{i}\right)\right) \cap \operatorname{Fix}(V) \cap\left(\bigcap_{i=0}^{\infty} \operatorname{VI}\left(C, A_{i}\right)\right) \neq \emptyset$. For arbitrarily given $x_{0} \in C$, let $\left\{x_{n}\right\}$ be the sequence generated by

$$
\begin{aligned}
x_{n+1}= & \alpha_{n} f\left(x_{n}\right)+\beta_{n} x_{n}+\gamma_{n} B_{n} x_{n} \\
& +\delta_{n} S_{n}((1-l) I+l V) x_{n}, \quad \forall n \geq 0,
\end{aligned}
$$

where $0<l<\alpha / \kappa^{2}$ and $\left\{\alpha_{n}\right\},\left\{\beta_{n}\right\},\left\{\gamma_{n}\right\}$, and $\left\{\delta_{n}\right\}$ are the sequences in $(0,1)$ such that $\alpha_{n}+\beta_{n}+\gamma_{n}+\delta_{n}=1$ for all $n \geq 0$. Suppose that the following conditions hold:

(i) $\lim _{n \rightarrow \infty} \alpha_{n}=0$ and $\sum_{n=0}^{\infty} \alpha_{n}=\infty$;

(ii) $\left\{\gamma_{n}\right\},\left\{\delta_{n}\right\} \subset[c, d]$ for some $c, d \in(0,1)$;

(iii) $\lim _{n \rightarrow \infty}\left(\left|\beta_{n}-\beta_{n-1}\right|+\left|\gamma_{n}-\gamma_{n-1}\right|+\left|\delta_{n}-\delta_{n-1}\right|\right)=0$;

(iv) $0<\lim \inf _{n \rightarrow \infty} \beta_{n} \leq \lim \sup _{n \rightarrow \infty} \beta_{n}<1$.

Assume that $\sum_{n=1}^{\infty} \sup _{x \in D}\left\|S_{n} x-S_{n-1} x\right\|<\infty$ for any bounded subset $D$ of $C$ and let $S$ be a mapping of $C$ into itself defined 
by $S x=\lim _{n \rightarrow \infty} S_{n} x$ for all $x \in C$ and suppose that $\operatorname{Fix}(S)=$ $\bigcap_{i=0}^{\infty} \operatorname{Fix}\left(S_{i}\right)$. Then, there hold the following:

(I) $\lim _{n \rightarrow \infty}\left\|x_{n+1}-x_{n}\right\|=0$;

(II) the sequence $\left\{x_{n}\right\}_{n=0}^{\infty}$ converges strongly to some $q \in$ $F$ which is the unique solution of the variational inequality problem (VIP)

$$
\langle(I-f) q, J(q-p)\rangle \leq 0, \quad \forall p \in F,
$$

provided $\beta_{n} \equiv \beta$ for some fixed $\beta \in(0,1)$.

Proof. In Theorem 31, we put $B_{1}=I-V, B_{2}=0$ and $\mu_{1}=l$ where $0<l<\alpha / \kappa^{2}$. Then GSVI (13) is equivalent to the VIP of finding $x^{*} \in C$ such that

$$
\left\langle B_{1} x^{*}, J\left(x-x^{*}\right)\right\rangle \geq 0, \quad \forall x \in C .
$$

In this case, $B_{1}: C \quad \rightarrow \quad X$ is $\alpha$-inverse strongly accretive. Repeating the same arguments as those in the proof of Corollary 25, we can infer that $\operatorname{Fix}(V)=\mathrm{VI}\left(C, B_{1}\right)$. Accordingly, we know that $F=\bigcap_{i=0}^{\infty} \operatorname{Fix}\left(T_{i}\right) \cap \Omega \cap A^{-1} 0=$ $\bigcap_{i=0}^{\infty} \operatorname{Fix}\left(T_{i}\right) \cap \operatorname{Fix}(V) \cap A^{-1} 0$, and

$$
\begin{aligned}
\Pi_{C}\left(I-\mu_{1} B_{1}\right) \Pi_{C}\left(I-\mu_{2} B_{2}\right) x_{n} \\
=\Pi_{C}\left(I-\mu_{1} B_{1}\right) x_{n} \\
=\Pi_{C}\left((1-l) x_{n}+l V x_{n}\right) \\
=((1-l) I+l V) x_{n} .
\end{aligned}
$$

So, scheme (178) reduces to (247). Therefore, the desired result follows from Theorem 31 .

Theorem 33. Let $C$ be a nonempty closed convex subset of a uniformly convex Banach space $X$ which has a uniformly Gateaux differentiable norm. Let $\Pi_{C}$ be a sunny nonexpansive retraction from $X$ onto $C$. Let $\left\{\rho_{n}\right\}_{n=0}^{\infty}$ be a sequence of positive numbers in $(0, b]$ for some $b \in(0,1)$ and $A_{i}: C \rightarrow X \xi_{i^{-}}$ strictly pseudocontractive and $\widehat{\alpha}_{i}$-strongly accretive with $\xi_{i}+$ $\widehat{\alpha}_{i} \geq 1$ for each $i=0,1, \ldots$ Define a mapping $G_{i}: C \rightarrow C$ by $\Pi_{C}\left(I-\lambda_{i} A_{i}\right) x=G_{i} x$ for all $x \in C$ and $i=0,1, \ldots$, where $1-\left(\xi_{i} /\left(1+\xi_{i}\right)\right)\left(1-\sqrt{\left(1-\widehat{\alpha}_{i}\right) / \xi_{i}}\right) \leq \lambda_{i} \leq 1$ for all $i=0,1, \ldots$ Let $B_{n}: C \rightarrow C$ be the $W$-mapping generated by $G_{n}, G_{n-1}, \ldots, G_{0}$ and $\rho_{n}, \rho_{n-1}, \ldots, \rho_{0}$. Let the mapping $B_{i}: C \rightarrow X$ be $\zeta_{i}$-strictly pseudocontractive and $\widehat{\beta}_{i}$-strongly accretive with $\zeta_{i}+\widehat{\beta}_{i} \geq 1$ for $i=1,2$. Let $f: C \rightarrow C$ be a contraction with coefficient $\rho \in(0,1)$. Let $\left\{S_{i}\right\}_{i=0}^{\infty}$ be a countable family of nonexpansive mappings of $C$ into itself such that $F=\left(\bigcap_{i=0}^{\infty} \operatorname{Fix}\left(S_{i}\right)\right) \cap$ $\Omega \cap\left(\bigcap_{i=0}^{\infty} \operatorname{VI}\left(C, A_{i}\right)\right) \neq \emptyset$, where $\Omega$ is the fixed point set of the mapping $G=\Pi_{C}\left(I-\mu_{1} B_{1}\right) \Pi_{C}\left(I-\mu_{2} B_{2}\right)$ with $1-\left(\zeta_{i} /(1+\right.$ $\left.\left.\zeta_{i}\right)\right)\left(1-\sqrt{\left(1-\widehat{\beta}_{i}\right) / \zeta_{i}}\right) \leq \mu_{i} \leq 1$ for $i=1$, 2. For arbitrarily given $x_{0} \in C$, let $\left\{x_{n}\right\}$ be the sequence generated by

$$
\begin{aligned}
x_{n+1}= & \sigma_{n} G x_{n}+\left(1-\sigma_{n}\right) \\
& \times\left[\alpha_{n} f\left(x_{n}\right)+\beta_{n} x_{n}+\gamma_{n} B_{n} x_{n}+\delta_{n} S_{n} G x_{n}\right], \quad \forall n \geq 0,
\end{aligned}
$$

where $\left\{\alpha_{n}\right\},\left\{\beta_{n}\right\},\left\{\gamma_{n}\right\},\left\{\delta_{n}\right\}$, and $\left\{\sigma_{n}\right\}$ are the sequences in $(0,1)$ such that $\alpha_{n}+\beta_{n}+\gamma_{n}+\delta_{n}=1$ for all $n \geq 0$. Suppose that the following conditions hold:

(i) $\lim _{n \rightarrow \infty} \alpha_{n}=0$ and $\sum_{n=0}^{\infty} \alpha_{n}=\infty$;

(ii) $\left\{\gamma_{n}\right\},\left\{\delta_{n}\right\} \subset[c, d]$ for some $c, d \in(0,1)$;

(iii) $\sum_{n=1}^{\infty}\left(\left|\sigma_{n}-\sigma_{n-1}\right|+\left|\alpha_{n}-\alpha_{n-1}\right|+\left|\beta_{n}-\beta_{n-1}\right|+\left|\gamma_{n}-\gamma_{n-1}\right|+\right.$ $\left.\left|\delta_{n}-\delta_{n-1}\right|\right)<\infty$;

(iv) $0<\lim \inf _{n \rightarrow \infty} \beta_{n} \leq \lim \sup _{n \rightarrow \infty} \beta_{n}<1$ and $0<$ $\lim \inf _{n \rightarrow \infty} \sigma_{n} \leq \lim \sup _{n \rightarrow \infty} \sigma_{n}<1$.

Assume that $\sum_{n=1}^{\infty} \sup _{x \in D}\left\|S_{n} x-S_{n-1} x\right\|<\infty$ for any bounded subset $D$ of $C$ and let $S$ be a mapping of $C$ into itself defined by $S x=\lim _{n \rightarrow \infty} S_{n} x$ for all $x \in C$ and suppose that $\operatorname{Fix}(S)=$ $\bigcap_{i=0}^{\infty} \operatorname{Fix}\left(S_{i}\right)$. Then, there hold the following:

(I) $\lim _{n \rightarrow \infty}\left\|x_{n+1}-x_{n}\right\|=0$;

(II) the sequence $\left\{x_{n}\right\}_{n=0}^{\infty}$ converges strongly to some $q \in$ $F$ which is the unique solution of the variational inequality problem (VIP)

$$
\langle(I-f) q, J(q-p)\rangle \leq 0, \quad \forall p \in F,
$$

$$
\text { provided } \beta_{n} \equiv \beta \text { for some fixed } \beta \in(0,1) .
$$

Proof. First of all, it is easy to see that (251) can be rewritten as follows:

$$
\begin{gathered}
y_{n}=\alpha_{n} f\left(x_{n}\right)+\beta_{n} x_{n}+\gamma_{n} B_{n} x_{n}+\delta_{n} S_{n} G x_{n}, \\
x_{x+1}=\sigma_{n} G x_{n}+\left(1-\sigma_{n}\right) y_{n}, \quad \forall n \geq 0 .
\end{gathered}
$$

Take a fixed $p \in F$ arbitrarily. Then, we obtain $p=G p, p=$ $B_{n} p$ and $S_{n} p=p$ for all $n \geq 0$. Thus, we get from (253)

$$
\begin{aligned}
\| y_{n}- & p \| \\
\leq & \alpha_{n}\left\|f\left(x_{n}\right)-p\right\|+\beta_{n}\left\|x_{n}-p\right\| \\
& +\gamma_{n}\left\|B_{n} x_{n}-p\right\|+\delta_{n}\left\|S_{n} G x_{n}-p\right\| \\
\leq & \alpha_{n}\left(\rho\left\|x_{n}-p\right\|+\|f(p)-p\|\right) \\
& +\beta_{n}\left\|x_{n}-p\right\|+\gamma_{n}\left\|x_{n}-p\right\|+\delta_{n}\left\|x_{n}-p\right\| \\
= & \left(1-\alpha_{n}(1-\rho)\right)\left\|x_{n}-p\right\|+\alpha_{n}\|f(p)-p\|,
\end{aligned}
$$


and hence

$$
\begin{aligned}
\| x_{n+1}- & p \| \\
\leq & \sigma_{n}\left\|G x_{n}-p\right\|+\left(1-\sigma_{n}\right)\left\|y_{n}-p\right\| \\
\leq & \sigma_{n}\left\|x_{n}-p\right\|+\left(1-\sigma_{n}\right) \\
& \times\left[\left(1-\alpha_{n}(1-\rho)\right)\left\|x_{n}-p\right\|+\alpha_{n}\|f(p)-p\|\right] \\
= & \left(1-\left(1-\sigma_{n}\right) \alpha_{n}(1-\rho)\right)\left\|x_{n}-p\right\| \\
& +\left(1-\sigma_{n}\right) \alpha_{n}\|f(p)-p\| \\
= & \left(1-\left(1-\sigma_{n}\right) \alpha_{n}(1-\rho)\right)\left\|x_{n}-p\right\| \\
& +\left(1-\sigma_{n}\right) \alpha_{n}(1-\rho) \frac{\|f(p)-p\|}{1-\rho} \\
\leq & \max \left\{\left\|x_{n}-p\right\|, \frac{\|f(p)-p\|}{1-\rho}\right\} .
\end{aligned}
$$

By induction, we have

$$
\left\|x_{n}-p\right\| \leq \max \left\{\left\|x_{0}-p\right\|, \frac{\|f(p)-p\|}{1-\rho}\right\}, \quad \forall n \geq 0 .
$$

which implies that $\left\{x_{n}\right\}$ is bounded and so are the sequences $\left\{y_{n}\right\},\left\{G x_{n}\right\}$ and $\left\{f\left(x_{n}\right)\right\}$.

Let us show that

$$
\lim _{n \rightarrow \infty}\left\|x_{n+1}-x_{n}\right\|=0 .
$$

As a matter of fact, observe that $y_{n}$ can be rewritten as follows:

$$
y_{n}=\beta_{n} x_{n}+\left(1-\beta_{n}\right) z_{n},
$$

where $z_{n}=\left(\alpha_{n} f\left(x_{n}\right)+\gamma_{n} B_{n} x_{n}+\delta_{n} S_{n} G x_{n}\right) /\left(1-\beta_{n}\right)$. Observe that

$$
\begin{aligned}
\| z_{n}- & z_{n-1} \| \\
= & \| \frac{\alpha_{n} f\left(x_{n}\right)+\gamma_{n} B_{n} x_{n}+\delta_{n} S_{n} G x_{n}}{1-\beta_{n}} \\
& \quad-\frac{\alpha_{n-1} f\left(x_{n-1}\right)+\gamma_{n-1} B_{n-1} x_{n-1}+\delta_{n-1} S_{n-1} G x_{n-1}}{1-\beta_{n-1}} \| \\
= & \left\|\frac{y_{n}-\beta_{n} x_{n}}{1-\beta_{n}}-\frac{y_{n-1}-\beta_{n-1} x_{n-1}}{1-\beta_{n-1}}\right\| \\
= & \| \frac{y_{n}-\beta_{n} x_{n}}{1-\beta_{n}}-\frac{y_{n-1}-\beta_{n-1} x_{n-1}}{1-\beta_{n}} \\
& +\frac{y_{n-1}-\beta_{n-1} x_{n-1}}{1-\beta_{n}}-\frac{y_{n-1}-\beta_{n-1} x_{n-1}}{1-\beta_{n-1}} \|
\end{aligned}
$$

$$
\begin{aligned}
\leq & \left\|\frac{y_{n}-\beta_{n} x_{n}}{1-\beta_{n}}-\frac{y_{n-1}-\beta_{n-1} x_{n-1}}{1-\beta_{n}}\right\| \\
& +\left\|\frac{y_{n-1}-\beta_{n-1} x_{n-1}}{1-\beta_{n}}-\frac{y_{n-1}-\beta_{n-1} x_{n-1}}{1-\beta_{n-1}}\right\| \\
= & \frac{1}{1-\beta_{n}}\left\|y_{n}-\beta_{n} x_{n}-\left(y_{n-1}-\beta_{n-1} x_{n-1}\right)\right\| \\
& +\left|\frac{1}{1-\beta_{n}}-\frac{1}{1-\beta_{n-1}}\right|\left\|y_{n-1}-\beta_{n-1} x_{n-1}\right\| \\
= & \frac{1}{1-\beta_{n}}\left\|y_{n}-\beta_{n} x_{n}-\left(y_{n-1}-\beta_{n-1} x_{n-1}\right)\right\| \\
& +\frac{\left|\beta_{n}-\beta_{n-1}\right|}{\left(1-\beta_{n-1}\right)\left(1-\beta_{n}\right)}\left\|y_{n-1}-\beta_{n-1} x_{n-1}\right\| \\
= & \frac{1}{1-\beta_{n}} \| \alpha_{n} f\left(x_{n}\right)+\gamma_{n} B_{n} x_{n}+\delta_{n} S_{n} G x_{n} \\
& \quad-\alpha_{n-1} f\left(x_{n-1}\right)-\gamma_{n-1} B_{n-1} x_{n-1} \\
& \quad-\delta_{n-1} S_{n-1} G x_{n-1} \| \\
& +\frac{\left|\beta_{n}-\beta_{n-1}\right|}{\left(1-\beta_{n-1}\right)\left(1-\beta_{n}\right)}\left\|y_{n-1}-\beta_{n-1} x_{n-1}\right\| . \\
& +\beta_{n}-\beta_{n-1} \mid \\
& +\delta_{n}\left\|S_{n} G x_{n}-S_{n-1} G x_{n-1}\right\| \\
\leq & \frac{1}{\left.1-\beta_{n-1}\right)\left(1-\beta_{n}\right)}\left\|y_{n-1}-\beta_{n-1} x_{n-1}\right\| f\left(x_{n-1} \mid\left\|B_{n-1} x_{n-1}\right\|\right. \\
& \left.+\left|\delta_{n}-\delta_{n-1}\right|\left\|S_{n-1} G x_{n-1}\right\|\right]
\end{aligned}
$$

On the other hand, repeating the same arguments as those of (52) and (54) in the proof of Theorem 24, we can deduce that for all $n \geq 1$

$$
\begin{aligned}
& \left\|S_{n} G x_{n}-S_{n-1} G x_{n-1}\right\| \\
& \quad \leq\left\|x_{n}-x_{n-1}\right\|+\left\|S_{n} G x_{n-1}-S_{n-1} G x_{n-1}\right\|, \\
& \left\|B_{n} x_{n}-B_{n-1} x_{n-1}\right\| \leq\left\|x_{n}-x_{n-1}\right\|+M \prod_{i=0}^{n-1} \lambda_{i},
\end{aligned}
$$

for some constant $M>0$. Taking into account $0<$ $\liminf _{n \rightarrow \infty} \beta_{n} \leq \limsup _{n \rightarrow \infty} \beta_{n}<1$, we may assume, 
without loss of generality, that $\left\{\beta_{n}\right\} \subset[\widehat{c}, \widehat{d}]$. Utilizing (259)(260) we have

$$
\begin{aligned}
& \left\|z_{n}-z_{n-1}\right\| \\
& \leq \frac{1}{1-\beta_{n}} \\
& \times\left\{\alpha_{n} \rho\left\|x_{n}-x_{n-1}\right\|+\gamma_{n}\left[\left\|x_{n}-x_{n-1}\right\|+M \prod_{i=0}^{n-1} \lambda_{i}\right]\right. \\
& +\delta_{n}\left[\left\|x_{n}-x_{n-1}\right\|+\left\|S_{n} G x_{n-1}-S_{n-1} G x_{n-1}\right\|\right] \\
& +\left|\alpha_{n}-\alpha_{n-1}\right|\left\|f\left(x_{n-1}\right)\right\|+\left|\gamma_{n}-\gamma_{n-1}\right| \| B_{n-1} x_{n-1}|| \\
& \left.+\left|\delta_{n}-\delta_{n-1}\right| \| S_{n-1} G x_{n-1}||\right\} \\
& +\frac{\left|\beta_{n}-\beta_{n-1}\right|}{\left(1-\beta_{n-1}\right)\left(1-\beta_{n}\right)}\left\|y_{n-1}-\beta_{n-1} x_{n-1}\right\| \\
& =\frac{1}{1-\beta_{n}}\left\{\left(1-\beta_{n}-\alpha_{n}(1-\rho)\right)\left\|x_{n}-x_{n-1}\right\|\right. \\
& +\gamma_{n} M \prod_{i=0}^{n-1} \lambda_{i}+\delta_{n}\left\|S_{n} G x_{n-1}-S_{n-1} G x_{n-1}\right\| \\
& +\left|\alpha_{n}-\alpha_{n-1}\right|\left\|f\left(x_{n-1}\right)\right\| \\
& +\left|\gamma_{n}-\gamma_{n-1}\right||| B_{n-1} x_{n-1}|| \\
& \left.+\left|\delta_{n}-\delta_{n-1}\right|\left\|S_{n-1} G x_{n-1}\right\|\right\} \\
& +\frac{\left|\beta_{n}-\beta_{n-1}\right|}{\left(1-\beta_{n-1}\right)\left(1-\beta_{n}\right)}\left\|y_{n-1}-\beta_{n-1} x_{n-1}\right\| \\
& =\left(1-\frac{\alpha_{n}(1-\rho)}{1-\beta_{n}}\right)\left\|x_{n}-x_{n-1}\right\|+\frac{\gamma_{n} M}{1-\beta_{n}} \prod_{i=0}^{n-1} \lambda_{i} \\
& +\frac{\delta_{n}}{1-\beta_{n}}\left\|S_{n} G x_{n-1}-S_{n-1} G x_{n-1}\right\| \\
& +\frac{1}{1-\beta_{n}}\left[\left|\alpha_{n}-\alpha_{n-1}\right|\left\|f\left(x_{n-1}\right)\right\|+\left|\gamma_{n}-\gamma_{n-1}\right|\right. \\
& \left.\times\left\|B_{n-1} x_{n-1}\right\|+\left|\delta_{n}-\delta_{n-1}\right|\left\|S_{n-1} G x_{n-1}\right\|\right] \\
& +\frac{\left|\beta_{n}-\beta_{n-1}\right|}{\left(1-\beta_{n-1}\right)\left(1-\beta_{n}\right)}\left\|y_{n-1}-\beta_{n-1} x_{n-1}\right\| \\
& \leq\left(1-\frac{\alpha_{n}(1-\rho)}{1-\beta_{n}}\right)\left\|x_{n}-x_{n-1}\right\|+M \prod_{i=0}^{n-1} \lambda_{i} \\
& +\left\|S_{n} G x_{n-1}-S_{n-1} G x_{n-1}\right\|+\frac{1}{1-\beta_{n}}
\end{aligned}
$$

$$
\begin{aligned}
& \times\left[\left|\alpha_{n}-\alpha_{n-1}\right|\left\|f\left(x_{n-1}\right)\right\|\right. \\
& +\left|\gamma_{n}-\gamma_{n-1}\right|\left\|B_{n-1} x_{n-1}\right\| \\
& \left.+\left|\delta_{n}-\delta_{n-1}\right|\left\|S_{n-1} G x_{n-1}\right\|\right]+\frac{\left|\beta_{n}-\beta_{n-1}\right|}{\left(1-\beta_{n-1}\right)\left(1-\beta_{n}\right)} \\
& \times\left\|\alpha_{n-1} f\left(x_{n-1}\right)+\gamma_{n-1} B_{n-1} x_{n-1}+\delta_{n-1} S_{n-1} G x_{n-1}\right\| \\
& \leq\left(1-\frac{\alpha_{n}(1-\rho)}{1-\beta_{n}}\right)\left\|x_{n}-x_{n-1}\right\| \\
& +M_{1}\left[\prod_{i=0}^{n-1} \lambda_{i}+\left|\alpha_{n}-\alpha_{n-1}\right|+\left|\beta_{n}-\beta_{n-1}\right|+\left|\gamma_{n}-\gamma_{n-1}\right|\right. \\
& \left.\quad+\left|\delta_{n}-\delta_{n-1}\right|\right]+\left\|S_{n} G x_{n-1}-S_{n-1} G x_{n-1}\right\|,
\end{aligned}
$$

where $\sup _{n \geq 0}\left\{\left(1 /(1-\widehat{d})^{2}\right)\left(\left\|f\left(x_{n}\right)\right\|+\left\|B_{n} x_{n}\right\|+\left\|S_{n} G x_{n}\right\|+\right.\right.$ $M)\} \leq M_{1}$ for some $M_{1}>0$. In the meantime, observe that

$$
\begin{aligned}
x_{n+1}-x_{n}= & \sigma_{n}\left(G x_{n}-G x_{n-1}\right)+\left(\sigma_{n}-\sigma_{n-1}\right)\left(G x_{n-1}-z_{n-1}\right) \\
& +\left(1-\sigma_{n}\right)\left(z_{n}-z_{n-1}\right) .
\end{aligned}
$$

This together with (261), implies that

$$
\begin{aligned}
& \left\|x_{n+1}-x_{n}\right\| \\
& \leq \sigma_{n}\left\|G x_{n}-G x_{n-1}\right\|+\left|\sigma_{n}-\sigma_{n-1}\right|\left\|G x_{n-1}-z_{n-1}\right\| \\
& \quad+\left(1-\sigma_{n}\right)\left\|z_{n}-z_{n-1}\right\| \\
& \leq \sigma_{n}\left\|x_{n}-x_{n-1}\right\|+\left|\sigma_{n}-\sigma_{n-1}\right|\left\|G x_{n-1}-z_{n-1}\right\| \\
& \quad+\left(1-\sigma_{n}\right)\left\{\left(1-\frac{\alpha_{n}(1-\rho)}{1-\beta_{n}}\right)\left\|x_{n}-x_{n-1}\right\|\right. \\
& +M_{1}\left[\prod_{i=0}^{n-1} \lambda_{i}+\left|\alpha_{n}-\alpha_{n-1}\right|+\left|\beta_{n}-\beta_{n-1}\right|\right. \\
& \left.+\left|\gamma_{n}-\gamma_{n-1}\right|+\left|\delta_{n}-\delta_{n-1}\right|\right] \\
& \left.\quad+\left(1-\frac{\left(1-\sigma_{n}\right) \alpha_{n}(1-\rho)}{1-\beta_{n}}\right)\left\|x_{n-1}-S_{n-1} G x_{n-1}\right\|\right\} \\
& +\left|\sigma_{n}-\sigma_{n-1}\right|\left\|G x_{n-1}-z_{n-1}\right\| \\
& +M_{1}\left[\prod_{i=0}^{n-1} \lambda_{i}+\left|\alpha_{n}-\alpha_{n-1}\right|+\left|\beta_{n}-\beta_{n-1}\right|\right. \\
& \left.+\left|\gamma_{n}-\gamma_{n-1}\right|+\left|\delta_{n}-\delta_{n-1}\right|\right]
\end{aligned}
$$




$$
\begin{aligned}
& +\left\|S_{n} G x_{n-1}-S_{n-1} G x_{n-1}\right\| \\
& \leq\left(1-\frac{\left(1-\sigma_{n}\right) \alpha_{n}(1-\rho)}{1-\beta_{n}}\right)\left\|x_{n}-x_{n-1}\right\| \\
& +M_{2}\left[\prod_{i=0}^{n-1} \lambda_{i}+\left|\sigma_{n}-\sigma_{n-1}\right|+\left|\alpha_{n}-\alpha_{n-1}\right|\right. \\
& \left.\quad+\left|\beta_{n}-\beta_{n-1}\right|+\left|\gamma_{n}-\gamma_{n-1}\right|+\left|\delta_{n}-\delta_{n-1}\right|\right] \\
& +\left\|S_{n} G x_{n-1}-S_{n-1} G x_{n-1}\right\|,
\end{aligned}
$$

where $\sup _{n \geq 0}\left\{M_{1}+\left\|G x_{n}-z_{n}\right\|\right\} \leq M_{2}$ for some $M_{2}>0$. Since $\sum_{n=0}^{\infty} \alpha_{n}=\infty$ and $\left(1-\sigma_{n}\right) \alpha_{n}(1-\rho) /\left(1-\beta_{n}\right) \geq\left(1-\sigma_{n}\right) \alpha_{n}(1-\rho)$, we obtain from conditions (i) and (iv) that $\sum_{n=0}^{\infty}\left(\left(1-\sigma_{n}\right) \alpha_{n}(1-\right.$ $\left.\rho) /\left(1-\beta_{n}\right)\right)=\infty$. Thus, applying Lemma 7 to $(263)$, we deduce from condition (iii) and the assumption on $\left\{S_{n}\right\}$ that (noting that $0<\lambda_{i} \leq b<1$, for all $i \geq 0$ )

$$
\lim _{n \rightarrow \infty}\left\|x_{n+1}-x_{n}\right\|=0
$$

Next, we show that $\left\|x_{n}-G x_{n}\right\| \rightarrow 0$ as $n \rightarrow \infty$. Indeed, according to Lemma 8 we have from (253)

$$
\begin{aligned}
\| y_{n}- & p \|^{2} \\
= & \| \alpha_{n}\left(f\left(x_{n}\right)-f(p)\right)+\beta_{n}\left(x_{n}-p\right)+\gamma_{n}\left(B_{n} x_{n}-p\right) \\
& +\delta_{n}\left(S_{n} G x_{n}-p\right)+\alpha_{n}(f(p)-p) \|^{2} \\
\leq & \| \alpha_{n}\left(f\left(x_{n}\right)-f(p)\right)+\beta_{n}\left(x_{n}-p\right) \\
& +\gamma_{n}\left(B_{n} x_{n}-p\right)+\delta_{n}\left(S_{n} G x_{n}-p\right) \|^{2} \\
& +2 \alpha_{n}\left\langle f(p)-p, J\left(x_{n+1}-p\right)\right\rangle \\
\leq & \alpha_{n}\left\|f\left(x_{n}\right)-f(p)\right\|^{2}+\beta_{n}\left\|x_{n}-p\right\|^{2} \\
& +\gamma_{n}\left\|B_{n} x_{n}-p\right\|^{2}+\delta_{n}\left\|S_{n} G x_{n}-p\right\|^{2} \\
& +2 \alpha_{n}\left\langle f(p)-p, J\left(x_{n+1}-p\right)\right\rangle \\
\leq & \alpha_{n} \rho\left\|x_{n}-p\right\|^{2}+\beta_{n}\left\|x_{n}-p\right\|^{2}+\gamma_{n}\left\|x_{n}-p\right\|^{2} \\
& +\delta_{n}\left\|x_{n}-p\right\|^{2}+2 \alpha_{n}\|f(p)-p\|\left\|x_{n+1}-p\right\| \\
= & \left(1-\alpha_{n}(1-\rho)\right)\left\|x_{n}-p\right\|^{2} \\
& +2 \alpha_{n}\|f(p)-p\|\left\|x_{n+1}-p\right\| \\
\leq & \left\|x_{n}-p\right\|^{2}+2 \alpha_{n}\|f(p)-p\|\left\|x_{n+1}-p\right\| .
\end{aligned}
$$

Utilizing Lemma 15 we get from (253) and (265)

$$
\begin{aligned}
&\left\|x_{n+1}-p\right\|^{2} \\
&=\left\|\sigma_{n}\left(G x_{n}-p\right)+\left(1-\sigma_{n}\right)\left(y_{n}-p\right)\right\|^{2} \\
& \leq \sigma_{n}\left\|G x_{n}-p\right\|^{2}+\left(1-\sigma_{n}\right)\left\|y_{n}-p\right\|^{2} \\
&-\sigma_{n}\left(1-\sigma_{n}\right) g\left(\left\|G x_{n}-y_{n}\right\|\right) \\
& \leq \sigma_{n}\left\|x_{n}-p\right\|^{2}+\left(1-\sigma_{n}\right) \\
& \times\left[\left\|x_{n}-p\right\|^{2}+2 \alpha_{n}\|f(p)-p\|\left\|x_{n+1}-p\right\|\right] \\
&-\sigma_{n}\left(1-\sigma_{n}\right) g\left(\left\|G x_{n}-y_{n}\right\|\right) \\
& \leq\left\|x_{n}-p\right\|^{2}+2 \alpha_{n}\|f(p)-p\|\left\|x_{n+1}-p\right\| \\
&-\sigma_{n}\left(1-\sigma_{n}\right) g\left(\left\|G x_{n}-y_{n}\right\|\right),
\end{aligned}
$$

which hence yields

$$
\begin{aligned}
\sigma_{n}(1- & \left.\sigma_{n}\right) g\left(\left\|G x_{n}-y_{n}\right\|\right) \\
\leq & \left\|x_{n}-p\right\|^{2}-\left\|x_{n+1}-p\right\|^{2} \\
& +2 \alpha_{n}\|f(p)-p\|\left\|x_{n+1}-p\right\| \\
\leq & \left(\left\|x_{n}-p\right\|+\left\|x_{n+1}-p\right\|\right)\left\|x_{n}-x_{n+1}\right\| \\
& +2 \alpha_{n}\|f(p)-p\|\left\|x_{n+1}-p\right\| .
\end{aligned}
$$

Since $\alpha_{n} \rightarrow 0$ and $\left\|x_{n+1}-x_{n}\right\| \rightarrow 0$, from condition (iv) and the boundedness of $\left\{x_{n}\right\}$, it follows that

$$
\lim _{n \rightarrow \infty} g\left(\left\|G x_{n}-y_{n}\right\|\right)=0 .
$$

Utilizing the properties of $g$, we have

$$
\lim _{n \rightarrow \infty}\left\|G x_{n}-y_{n}\right\|=0
$$

which, together with (253) and (257), implies that

$$
\begin{aligned}
\| x_{n}- & y_{n} \| \\
\leq & \left\|x_{n}-x_{n+1}\right\|+\left\|x_{n+1}-y_{n}\right\| \\
= & \left\|x_{n}-x_{n+1}\right\|+\sigma_{n}\left\|G x_{n}-y_{n}\right\| \longrightarrow 0 \\
& \text { as } n \longrightarrow \infty .
\end{aligned}
$$

That is,

$$
\lim _{n \rightarrow \infty}\left\|x_{n}-y_{n}\right\|=0
$$

Since

$$
\left\|x_{n}-G x_{n}\right\| \leq\left\|x_{n}-y_{n}\right\|+\left\|y_{n}-G x_{n}\right\|,
$$

it immediately follows from (269) and (271) that

$$
\lim _{n \rightarrow \infty}\left\|x_{n}-G x_{n}\right\|=0 .
$$


On the other hand, observe that $y_{n}$ can be rewritten as follows:

$$
\begin{aligned}
y_{n} & =\alpha_{n} f\left(x_{n}\right)+\beta_{n} x_{n}+\gamma_{n} B_{n} x_{n}+\delta_{n} S_{n} G x_{n} \\
& =\alpha_{n} f\left(x_{n}\right)+\beta_{n} x_{n}+\left(\gamma_{n}+\delta_{n}\right) \frac{\gamma_{n} B_{n} x_{n}+\delta_{n} S_{n} G x_{n}}{\gamma_{n}+\delta_{n}} \\
& =\alpha_{n} f\left(x_{n}\right)+\beta_{n} x_{n}+e_{n} \widehat{z}_{n},
\end{aligned}
$$

where $e_{n}=\gamma_{n}+\delta_{n}$ and $\widehat{z}_{n}=\left(\gamma_{n} B_{n} x_{n}+\delta_{n} S_{n} G x_{n}\right) /\left(\gamma_{n}+\delta_{n}\right)$. Utilizing Lemma 11, we have

$$
\begin{aligned}
\| y_{n}- & p \|^{2} \\
= & \left\|\alpha_{n}\left(f\left(x_{n}\right)-p\right)+\beta_{n}\left(x_{n}-p\right)+e_{n}\left(\widehat{z}_{n}-p\right)\right\|^{2} \\
\leq & \alpha_{n}\left\|f\left(x_{n}\right)-p\right\|^{2}+\beta_{n}\left\|x_{n}-p\right\|^{2} \\
& +e_{n}\left\|\widehat{z}_{n}-p\right\|^{2}-\beta_{n} e_{n} g_{1}\left(\left\|\widehat{z}_{n}-x_{n}\right\|\right) \\
= & \alpha_{n}\left\|f\left(x_{n}\right)-p\right\|^{2}+\beta_{n}\left\|x_{n}-p\right\|^{2} \\
& -\beta_{n} e_{n} g_{1}\left(\left\|\bar{z}_{n}-x_{n}\right\|\right) \\
& +e_{n}\left\|\frac{\gamma_{n} B_{n} x_{n}+\delta_{n} S_{n} G x_{n}}{\gamma_{n}+\delta_{n}}-p\right\|^{2} \\
= & \alpha_{n}\left\|f\left(x_{n}\right)-p\right\|^{2}+\beta_{n}\left\|x_{n}-p\right\|^{2} \\
& -\beta_{n} e_{n} g_{1}\left(\left\|\widehat{z}_{n}-x_{n}\right\|\right) \\
& +e_{n}\left\|\frac{\gamma_{n}}{\gamma_{n}+\delta_{n}}\left(B_{n} x_{n}-p\right)+\frac{\delta_{n}}{\gamma_{n}+\delta_{n}}\left(S_{n} G x_{n}-p\right)\right\|^{2} \\
\leq & \alpha_{n}\left\|f\left(x_{n}\right)-p\right\|^{2}+\beta_{n}\left\|x_{n}-p\right\|^{2} \\
& -\beta_{n} e_{n} g_{1}\left(\left\|\bar{z}_{n}-x_{n}\right\|\right) \\
& +e_{n}\left[\frac{\gamma_{n}}{\gamma_{n}+\delta_{n}}\left\|B_{n} x_{n}-p\right\|^{2}+\frac{\delta_{n}}{\gamma_{n}+\delta_{n}}\left\|S_{n} G x_{n}-p\right\|^{2}\right] \\
\leq \alpha_{n} & \left\|f\left(x_{n}\right)-p\right\|^{2}+\beta_{n}\left\|x_{n}-p\right\|^{2} \\
& -\beta_{n} e_{n} g_{1}\left(\left\|\bar{z}_{n}-x_{n}\right\|\right) \\
& +e_{n}\left[\frac{\gamma_{n}}{\gamma_{n}+\delta_{n}}\left\|x_{n}-p\right\|^{2}+\frac{\delta_{n}}{\gamma_{n}+\delta_{n}}\left\|x_{n}-p\right\|^{2}\right] \\
= & \alpha_{n}\left\|f\left(x_{n}\right)-p\right\|^{2}+\left(1-\alpha_{n}\right)\left\|x_{n}-p\right\|^{2} \\
& -\beta_{n} e_{n} g_{1}\left(\left\|\bar{z}_{n}-x_{n}\right\|\right) \\
\leq & \alpha_{n}\left\|f\left(x_{n}\right)-p\right\|^{2}+\left\|x_{n}-p\right\|^{2} \\
& -\beta_{n} e_{n} g_{1}\left(\left\|\widehat{z}_{n}-x_{n}\right\|\right), \\
&
\end{aligned}
$$

which hence implies that

$$
\begin{aligned}
& \beta_{n} e_{n} g_{1}\left(\left\|\widehat{z}_{n}-x_{n}\right\|\right) \\
& \quad \leq \alpha_{n}\left\|f\left(x_{n}\right)-p\right\|^{2}+\left\|x_{n}-p\right\|^{2}-\left\|y_{n}-p\right\|^{2} \\
& \quad \leq \alpha_{n}\left\|f\left(x_{n}\right)-p\right\|^{2}+\left(\left\|x_{n}-p\right\|+\left\|y_{n}-p\right\|\right)\left\|x_{n}-y_{n}\right\| .
\end{aligned}
$$

Utilizing (271), conditions (i), (ii), (iv), and the boundedness of $\left\{x_{n}\right\},\left\{y_{n}\right\}$ and $\left\{f\left(x_{n}\right)\right\}$, we get

$$
\lim _{n \rightarrow \infty} g_{1}\left(\left\|\widehat{z}_{n}-x_{n}\right\|\right)=0 .
$$

From the properties of $g_{1}$, we have

$$
\lim _{n \rightarrow \infty}\left\|\widehat{z}_{n}-x_{n}\right\|=0 .
$$

Utilizing Lemma 15 and the definition of $\widehat{z}_{n}$, we have

$$
\begin{aligned}
\| \bar{z}_{n}- & p \|^{2} \\
= & \left\|\frac{\gamma_{n} B_{n} x_{n}+\delta_{n} S_{n} G x_{n}}{\gamma_{n}+\delta_{n}}-p\right\|^{2} \\
= & \left\|\frac{\gamma_{n}}{\gamma_{n}+\delta_{n}}\left(B_{n} x_{n}-p\right)+\frac{\delta_{n}}{\gamma_{n}+\delta_{n}}\left(S_{n} G x_{n}-p\right)\right\|^{2} \\
\leq & \frac{\gamma_{n}}{\gamma_{n}+\delta_{n}}\left\|B_{n} x_{n}-p\right\|^{2}+\frac{\delta_{n}}{\gamma_{n}+\delta_{n}}\left\|S_{n} G x_{n}-p\right\|^{2} \\
& -\frac{\gamma_{n} \delta_{n}}{\left(\gamma_{n}+\delta_{n}\right)^{2}} g_{2}\left(\left\|S_{n} G x_{n}-B_{n} x_{n}\right\|\right) \\
\leq & \left\|x_{n}-p\right\|^{2}-\frac{\gamma_{n} \delta_{n}}{\left(\gamma_{n}+\delta_{n}\right)^{2}} g_{2}\left(\left\|S_{n} G x_{n}-B_{n} x_{n}\right\|\right),
\end{aligned}
$$

which leads to

$$
\begin{aligned}
& \frac{\gamma_{n} \delta_{n}}{\left(\gamma_{n}+\delta_{n}\right)^{2}} g_{2}\left(\left\|S_{n} G x_{n}-B_{n} x_{n}\right\|\right) \\
& \quad \leq\left\|x_{n}-p\right\|^{2}-\left\|\widehat{z}_{n}-p\right\|^{2} \\
& \quad \leq\left(\left\|x_{n}-p\right\|+\left\|\widehat{z}_{n}-p\right\|\right)\left\|x_{n}-\widehat{z}_{n}\right\| .
\end{aligned}
$$

Since $\left\{x_{n}\right\}$ and $\left\{\widehat{z}_{n}\right\}$ are bounded, we deduce from (278) and condition (ii) that

$$
\lim _{n \rightarrow \infty} g_{2}\left(\left\|B_{n} x_{n}-S_{n} G x_{n}\right\|\right)=0 .
$$

From the properties of $g_{2}$, we have

$$
\lim _{n \rightarrow \infty}\left\|B_{n} x_{n}-S_{n} G x_{n}\right\|=0 .
$$

Furthermore, $y_{n}$ can also be rewritten as follows:

$$
\begin{aligned}
y_{n} & =\alpha_{n} f\left(x_{n}\right)+\beta_{n} x_{n}+\gamma_{n} B_{n} x_{n}+\delta_{n} S_{n} G x_{n} \\
& =\beta_{n} x_{n}+\gamma_{n} B_{n} x_{n}+\left(\alpha_{n}+\delta_{n}\right) \frac{\alpha_{n} f\left(x_{n}\right)+\delta_{n} S_{n} G x_{n}}{\alpha_{n}+\delta_{n}} \\
& =\beta_{n} x_{n}+\gamma_{n} B_{n} x_{n}+d_{n} \widetilde{z}_{n},
\end{aligned}
$$


where $d_{n}=\alpha_{n}+\delta_{n}$ and $\widetilde{z}_{n}=\left(\alpha_{n} f\left(x_{n}\right)+\delta_{n} S_{n} G x_{n}\right) /\left(\alpha_{n}+\delta_{n}\right)$. Utilizing Lemma 11 and the convexity of $\|\cdot\|^{2}$, we have

$$
\begin{aligned}
\| y_{n}- & p \|^{2} \\
= & \left\|\beta_{n}\left(x_{n}-p\right)+\gamma_{n}\left(B_{n} x_{n}-p\right)+d_{n}\left(\widetilde{z}_{n}-p\right)\right\|^{2} \\
\leq & \beta_{n}\left\|x_{n}-p\right\|^{2}+\gamma_{n}\left\|B_{n} x_{n}-p\right\|^{2} \\
& +d_{n}\left\|\tilde{z}_{n}-p\right\|^{2}-\beta_{n} \gamma_{n} g_{3}\left(\left\|x_{n}-B_{n} x_{n}\right\|\right) \\
= & \beta_{n}\left\|x_{n}-p\right\|^{2}+\gamma_{n}\left\|B_{n} x_{n}-p\right\|^{2} \\
& +d_{n}\left\|\frac{\alpha_{n} f\left(x_{n}\right)+\delta_{n} S_{n} G x_{n}}{\alpha_{n}+\delta_{n}}-p\right\|^{2} \\
& -\beta_{n} \gamma_{n} g_{3}\left(\left\|x_{n}-B_{n} x_{n}\right\|\right) \\
= & \beta_{n}\left\|x_{n}-p\right\|^{2}+\gamma_{n}\left\|B_{n} x_{n}-p\right\|^{2} \\
& +d_{n}\left\|\frac{\alpha_{n}}{\alpha_{n}+\delta_{n}}\left(f\left(x_{n}\right)-p\right)+\frac{\delta_{n}}{\alpha_{n}+\delta_{n}}\left(S_{n} G x_{n}-p\right)\right\|^{2} \\
& -\beta_{n} \gamma_{n} g_{3}\left(\left\|x_{n}-B_{n} x_{n}\right\|\right) \\
\leq & \beta_{n}\left\|x_{n}-p\right\|^{2}+\gamma_{n}\left\|x_{n}-p\right\|^{2} \\
& +d_{n}\left[\frac{\alpha_{n}}{\alpha_{n}+\delta_{n}}\left\|f\left(x_{n}\right)-p\right\|^{2}+\frac{\delta_{n}}{\alpha_{n}+\delta_{n}}\left\|S_{n} G x_{n}-p\right\|^{2}\right] \\
& -\beta_{n} \gamma_{n} g_{3}\left(\left\|x_{n}-B_{n} x_{n}\right\|\right) \\
\leq & \alpha_{n}\left\|f\left(x_{n}\right)-p\right\|^{2}+\left(\beta_{n}+\gamma_{n}\right)\left\|x_{n}-p\right\|^{2} \\
& +\delta_{n}\left\|x_{n}-p\right\|^{2}-\beta_{n} \gamma_{n} g_{3}\left(\left\|x_{n}-B_{n} x_{n}\right\|\right) \\
= & \alpha_{n}\left\|f\left(x_{n}\right)-p\right\|^{2}+\left(1-\alpha_{n}\right)\left\|x_{n}-p\right\|^{2} \\
& -\beta_{n} \gamma_{n} g_{3}\left(\left\|x_{n}-B_{n} x_{n}\right\|\right) \\
\leq & \alpha_{n}\left\|f\left(x_{n}\right)-p\right\|^{2}+\left\|x_{n}-p\right\|^{2} \\
& -\beta_{n} \gamma_{n} g_{3}\left(\left\|x_{n}-B_{n} x_{n}\right\|\right), \\
&
\end{aligned}
$$

which hence implies that

$$
\begin{aligned}
& \beta_{n} \gamma_{n} g_{3}\left(\left\|x_{n}-B_{n} x_{n}\right\|\right) \\
& \leq \alpha_{n}\left\|f\left(x_{n}\right)-p\right\|^{2}+\left\|x_{n}-p\right\|^{2}-\left\|y_{n}-p\right\|^{2} \\
& \leq \alpha_{n}\left\|f\left(x_{n}\right)-p\right\|^{2}+\left(\left\|x_{n}-p\right\|+\left\|y_{n}-p\right\|\right) \\
& \quad \times\left\|x_{n}-y_{n}\right\| .
\end{aligned}
$$

Utilizing (271), conditions (i), (ii), (iv), and the boundedness of $\left\{x_{n}\right\},\left\{y_{n}\right\}$ and $\left\{f\left(x_{n}\right)\right\}$, we get

$$
\lim _{n \rightarrow \infty} g_{3}\left(\left\|x_{n}-B_{n} x_{n}\right\|\right)=0 .
$$

From the properties of $g_{3}$, we have

$$
\lim _{n \rightarrow \infty}\left\|x_{n}-B_{n} x_{n}\right\|=0 .
$$

Thus, from (282) and (287), we get

$$
\begin{aligned}
\left\|x_{n}-S_{n} G x_{n}\right\| \leq & \left\|x_{n}-B_{n} x_{n}\right\| \\
& +\left\|B_{n} x_{n}-S_{n} G x_{n}\right\| \longrightarrow 0 \quad \text { as } n \longrightarrow \infty .
\end{aligned}
$$

That is,

$$
\lim _{n \rightarrow \infty}\left\|x_{n}-S_{n} G x_{n}\right\|=0 .
$$

Therefore, from Lemma 12, (273), and (289), it follows that

$$
\begin{aligned}
\| x_{n}- & S x_{n} \| \\
\leq & \left\|x_{n}-S_{n} G x_{n}\right\|+\left\|S_{n} G x_{n}-S_{n} x_{n}\right\|+\left\|S_{n} x_{n}-S x_{n}\right\| \\
\leq & \left\|x_{n}-S_{n} G x_{n}\right\|+\left\|G x_{n}-x_{n}\right\| \\
& +\left\|S_{n} x_{n}-S x_{n}\right\| \longrightarrow 0 \quad \text { as } n \longrightarrow \infty .
\end{aligned}
$$

That is,

$$
\lim _{n \rightarrow \infty}\left\|x_{n}-S x_{n}\right\|=0
$$

Suppose that $\beta_{n} \equiv \beta$ for some fixed $\beta \in(0,1)$ such that $\alpha_{n}+\beta+\gamma_{n}+\delta_{n}=1$ for all $n \geq 0$. Define a mapping $V x=$ $\left(1-\theta_{1}-\theta_{2}\right) S x+\theta_{1} B x+\theta_{2} G x$, where $\theta_{1}, \theta_{2} \in(0,1)$ are two constants with $\theta_{1}+\theta_{2}<1$. Then, by Lemmas 14 and 17, we have that $\operatorname{Fix}(V)=\operatorname{Fix}(S) \cap \operatorname{Fix}(B) \cap \operatorname{Fix}(G)=F$. For each $k \geq 1$, let $\left\{p_{k}\right\}$ be a unique element of $C$ such that

$$
p_{k}=\frac{1}{k} f\left(p_{k}\right)+\left(1-\frac{1}{k}\right) V p_{k} .
$$

From Lemma 13, we conclude that $p_{k} \rightarrow q \in \operatorname{Fix}(V)=F$ as $k \rightarrow \infty$. Repeating the same arguments as those of (81) in the proof of Theorem 24, we can conclude that for every $n, k$

$$
\begin{aligned}
\| y_{n}- & B p_{k} \| \\
\leq & \alpha_{n}\left\|f\left(x_{n}\right)-B p_{k}\right\|+\beta\left\|x_{n}-B p_{k}\right\| \\
& \quad+\gamma_{n}\left\|B_{n} x_{n}-B p_{k}\right\|+\delta_{n}\left\|S_{n} G x_{n}-B p_{k}\right\| \\
\leq & \theta_{n}+\beta\left\|x_{n}-B p_{k}\right\|+(1-\beta)\left\|x_{n}-p_{k}\right\|,
\end{aligned}
$$

where $\theta_{n}=\alpha_{n}\left\|f\left(x_{n}\right)-B p_{k}\right\|+(1-\beta)\left\|B_{n} p_{k}-B p_{k}\right\|+$ $\delta_{n}\left\|S_{n} G x_{n}-B_{n} x_{n}\right\|$. Since $\lim _{n \rightarrow \infty} \alpha_{n}=\lim _{n \rightarrow \infty}\left\|B_{n} p_{k}-B p_{k}\right\|=$ $\lim _{n \rightarrow \infty}\left\|S_{n} G x_{n}-B_{n} x_{n}\right\|=0$, we know that $\theta_{n} \rightarrow 0$ as 
$n \rightarrow \infty$. So, it immediately follows that

$$
\begin{aligned}
\| x_{n+1}- & B p_{k} \|^{2} \\
= & \left\|y_{n}-B p_{k}\right\|^{2}+\left\|x_{n+1}-y_{n}\right\| \\
& \times\left[2\left\|y_{n}-B p_{k}\right\|+\left\|x_{n+1}-y_{n}\right\|\right] \\
\leq & \left(\beta\left\|x_{n}-B p_{k}\right\|+(1-\beta)\left\|x_{n}-p_{k}\right\|\right)^{2} \\
& +\theta_{n}\left[2\left(\beta\left\|x_{n}-B p_{k}\right\|+(1-\beta)\left\|x_{n}-p_{k}\right\|\right)+\theta_{n}\right] \\
& +\left\|x_{n+1}-y_{n}\right\|\left[2\left\|y_{n}-B p_{k}\right\|+\left\|x_{n+1}-y_{n}\right\|\right] \\
= & \beta^{2}\left\|x_{n}-B p_{k}\right\|^{2}+(1-\beta)^{2}\left\|x_{n}-p_{k}\right\|^{2} \\
& +2 \beta(1-\beta)\left\|x_{n}-B p_{k}\right\|\left\|x_{n}-p_{k}\right\|+\tau_{n} \\
\leq & \beta^{2}\left\|x_{n}-B p_{k}\right\|^{2}+(1-\beta)^{2}\left\|x_{n}-p_{k}\right\|^{2} \\
& +\beta(1-\beta)\left(\left\|x_{n}-B p_{k}\right\|^{2}+\left\|x_{n}-p_{k}\right\|^{2}\right)+\tau_{n} \\
= & \beta\left\|x_{n}-B p_{k}\right\|^{2}+(1-\beta)\left\|x_{n}-p_{k}\right\|^{2}+\tau_{n},
\end{aligned}
$$

where $\tau_{n}=\theta_{n}\left[2\left(\beta\left\|x_{n}-B p_{k}\right\|+(1-\beta)\left\|x_{n}-p_{k}\right\|\right)+\theta_{n}\right]+\| x_{n+1}-$ $y_{n} \|\left[2\left\|y_{n}-B p_{k}\right\|+\left\|x_{n+1}-y_{n}\right\|\right] \rightarrow 0$ as $n \rightarrow \infty$.

For any Banach limit $\mu$, from (294), we derive

$$
\mu_{n}\left\|x_{n}-B p_{k}\right\|^{2}=\mu_{n}\left\|x_{n+1}-B p_{k}\right\|^{2} \leq \mu_{n}\left\|x_{n}-p_{k}\right\|^{2} \text {. }
$$

In addition, note that

$$
\begin{aligned}
\| x_{n}- & G p_{k} \|^{2} \\
\leq & \left\|x_{n}-G x_{n}+G x_{n}-G p_{k}\right\|^{2} \\
\leq & \left(\left\|x_{n}-G x_{n}\right\|+\left\|x_{n}-p_{k}\right\|\right)^{2} \\
= & \left\|x_{n}-p_{k}\right\|^{2}+\left\|x_{n}-G x_{n}\right\| \\
& \times\left(2\left\|x_{n}-p_{k}\right\|+\left\|x_{n}-G x_{n}\right\|\right), \\
\| x_{n}- & S p_{k} \|^{2} \\
\leq & \left\|x_{n}-S x_{n}+S x_{n}-S p_{k}\right\|^{2} \\
\leq & \left(\left\|x_{n}-S x_{n}\right\|+\left\|x_{n}-p_{k}\right\|\right)^{2} \\
= & \left\|x_{n}-p_{k}\right\|^{2}+\left\|x_{n}-S x_{n}\right\| \\
& \times\left(2\left\|x_{n}-p_{k}\right\|+\left\|x_{n}-S x_{n}\right\|\right) .
\end{aligned}
$$

It is easy to see from (273) and (291) that

$$
\begin{aligned}
& \mu_{n}\left\|x_{n}-G p_{k}\right\|^{2} \leq \mu_{n}\left\|x_{n}-p_{k}\right\|^{2}, \\
& \mu_{n}\left\|x_{n}-S p_{k}\right\|^{2} \leq \mu_{n}\left\|x_{n}-p_{k}\right\|^{2} .
\end{aligned}
$$

Utilizing (295) and (297), we deduce that

$$
\begin{aligned}
\mu_{n}\left\|x_{n}-V p_{k}\right\|^{2} & \\
= & \mu_{n} \|\left(1-\theta_{1}-\theta_{2}\right)\left(x_{n}-S p_{k}\right) \\
& \quad+\theta_{1}\left(x_{n}-B p_{k}\right)+\theta_{2}\left(x_{n}-G p_{k}\right) \|^{2} \\
\leq & \left(1-\theta_{1}-\theta_{2}\right) \mu_{n}\left\|x_{n}-S p_{k}\right\|^{2}+\theta_{1} \mu_{n}\left\|x_{n}-B p_{k}\right\|^{2} \\
& +\theta_{2} \mu_{n}\left\|x_{n}-G p_{k}\right\|^{2} \\
\leq & \mu_{n}\left\|x_{n}-p_{k}\right\|^{2} .
\end{aligned}
$$

Repeating the same arguments as those of (99) in the proof of Theorem 24, we can obtain that

$$
\frac{1}{2 k} \mu_{n}\left\|x_{n}-p_{k}\right\|^{2} \geq \mu_{n}\left\langle f\left(p_{k}\right)-p_{k}, J\left(x_{n}-p_{k}\right)\right\rangle .
$$

Since $p_{k} \rightarrow q \in \operatorname{Fix}(V)=F$ as $k \rightarrow \infty$, by the uniform Gateaux differentiability of the norm of $X$ we have

$$
\mu_{n}\left\langle f(q)-q, J\left(x_{n}-q\right)\right\rangle \leq 0 .
$$

On the other hand, from (257) and the norm-to-weak* uniform continuity of $J$ on bounded subsets of $X$, it follows that

$$
\lim _{n \rightarrow \infty}\left|\left\langle f(q)-q, J\left(x_{n+1}-q\right)\right\rangle-\left\langle f(q)-q, J\left(x_{n}-q\right)\right\rangle\right|=0 .
$$

So, utilizing Lemma 18, we deduce from (300) and (301) that

$$
\limsup _{n \rightarrow \infty}\left\langle f(q)-q, J\left(x_{n}-q\right)\right\rangle \leq 0
$$

which together with (271) and the norm-to-weak ${ }^{*}$ uniform continuity of $J$ on bounded subsets of $X$, implies that

$$
\limsup _{n \rightarrow \infty}\left\langle f(q)-q, J\left(y_{n}-q\right)\right\rangle \leq 0
$$

Finally, let us show that $x_{n} \rightarrow q$ as $n \rightarrow \infty$. Utilizing Lemma 8 (i), from (253) and the convexity of $\|\cdot\|$, we get

$$
\begin{aligned}
& \left\|y_{n}-q\right\|^{2} \\
& \leq \| \alpha_{n}\left(f\left(x_{n}\right)-f(q)\right)+\beta_{n}\left(x_{n}-q\right) \\
& \quad+\gamma_{n}\left(B_{n} x_{n}-q\right)+\delta_{n}\left(S_{n} G x_{n}-q\right) \|^{2} \\
& \quad+2 \alpha_{n}\left\langle f(q)-q, J\left(y_{n}-q\right)\right\rangle
\end{aligned}
$$




$$
\begin{aligned}
\leq & \alpha_{n}\left\|f\left(x_{n}\right)-f(q)\right\|^{2}+\beta_{n}\left\|x_{n}-q\right\|^{2} \\
& +\gamma_{n}\left\|B_{n} x_{n}-q\right\|^{2}+\delta_{n}\left\|S_{n} G x_{n}-q\right\|^{2} \\
& +2 \alpha_{n}\left\langle f(q)-q, J\left(y_{n}-q\right)\right\rangle \\
\leq & \alpha_{n} \rho\left\|x_{n}-q\right\|^{2}+\beta_{n}\left\|x_{n}-q\right\|^{2} \\
& +\gamma_{n}\left\|x_{n}-q\right\|^{2}+\delta_{n}\left\|x_{n}-q\right\|^{2} \\
& +2 \alpha_{n}\left\langle f(q)-q, J\left(y_{n}-q\right)\right\rangle \\
= & \left(1-\alpha_{n}(1-\rho)\right)\left\|x_{n}-q\right\|^{2} \\
& +2 \alpha_{n}\left\langle f(q)-q, J\left(y_{n}-q\right)\right\rangle,
\end{aligned}
$$

and hence

$$
\begin{aligned}
\left\|x_{n+1}-q\right\|^{2} & \\
\leq & \sigma_{n}\left\|G x_{n}-q\right\|^{2}+\left(1-\sigma_{n}\right)\left\|y_{n}-q\right\|^{2} \\
\leq & \sigma_{n}\left\|x_{n}-q\right\|^{2}+\left(1-\sigma_{n}\right) \\
& \times\left[\left(1-\alpha_{n}(1-\rho)\right)\left\|x_{n}-q\right\|^{2}\right. \\
& \left.\quad+2 \alpha_{n}\left\langle f(q)-q, J\left(y_{n}-q\right)\right\rangle\right] \\
= & {\left[1-\left(1-\sigma_{n}\right) \alpha_{n}(1-\rho)\right]\left\|x_{n}-q\right\|^{2} } \\
& +2\left(1-\sigma_{n}\right) \alpha_{n}\left\langle f(q)-q, J\left(y_{n}-q\right)\right\rangle \\
= & {\left[1-\left(1-\sigma_{n}\right) \alpha_{n}(1-\rho)\right]\left\|x_{n}-q\right\|^{2} } \\
& +\left(1-\sigma_{n}\right) \alpha_{n}(1-\rho) \frac{2\left\langle f(q)-q, J\left(y_{n}-q\right)\right\rangle}{1-\rho} .
\end{aligned}
$$

From conditions (i) and (iv), it is easy to see that $\sum_{n=0}^{\infty}(1-$ $\left.\sigma_{n}\right) \alpha_{n}(1-\rho)=\infty$. Applying Lemma 7 to (305), we infer that $x_{n} \rightarrow q$ as $n \rightarrow \infty$. This completes the proof.

Corollary 34. Let $C$ be a nonempty closed convex subset of a uniformly convex Banach space $X$ which has a uniformly Gateaux differentiable norm. Let $\Pi_{C}$ be a sunny nonexpansive retraction from $X$ onto $C$. Let $\left\{\rho_{n}\right\}_{n=0}^{\infty}$ be a sequence of positive numbers in $(0, b]$ for some $b \in(0,1)$ and $A_{i}: C \rightarrow X \xi_{i^{-}}$ strictly pseudocontractive and $\widehat{\alpha}_{i}$-strongly accretive with $\xi_{i}+$ $\widehat{\alpha}_{i} \geq 1$ for each $i=0,1, \ldots$. Define a mapping $G_{i}: C \rightarrow C$ by $\Pi_{C}\left(I-\lambda_{i} A_{i}\right) x=G_{i} x$ for all $x \in C$ and $i=0,1, \ldots$, where $1-\left(\xi_{i} /\left(1+\xi_{i}\right)\right)\left(1-\sqrt{\left(1-\widehat{\alpha}_{i}\right) / \xi_{i}}\right) \leq \lambda_{i} \leq 1$ for all $i=0,1, \ldots$ Let $B_{n}: C \rightarrow C$ be the $W$-mapping generated by $G_{n}, G_{n-1}, \ldots, G_{0}$ and $\rho_{n}, \rho_{n-1}, \ldots, \rho_{0}$. Let $V: C \rightarrow C$ be a self-mapping such that $I-V: C \rightarrow X$ is $\zeta$-strictly pseudocontractive and $\theta$ strongly accretive with $\theta+\zeta \geq 1$. Let $f: C \rightarrow C$ be a contraction with coefficient $\rho \in(0,1)$. Let $\left\{S_{i}\right\}_{i=0}^{\infty}$ be a countable family of nonexpansive mappings of $C$ into itself such that $F=\left(\bigcap_{i=0}^{\infty} \operatorname{Fix}\left(S_{i}\right)\right) \cap \operatorname{Fix}(V) \cap\left(\bigcap_{i=0}^{\infty} \operatorname{VI}\left(C, A_{i}\right)\right) \neq \emptyset$. For arbitrarily given $x_{0} \in C$, let $\left\{x_{n}\right\}$ be the sequence generated by

$$
\begin{aligned}
& x_{n+1} \\
& =\sigma_{n}((1-l) I+l V) x_{n}+\left(1-\sigma_{n}\right) \\
& \quad \times\left[\alpha_{n} f\left(x_{n}\right)+\beta_{n} x_{n}+\gamma_{n} B_{n} x_{n}+\delta_{n} S_{n}((1-l) I+l V) x_{n}\right], \\
& \forall n \geq 0,
\end{aligned}
$$

where $1-(\zeta /(1+\zeta))(1-\sqrt{(1-\theta) / \zeta}) \leq l \leq 1$ and $\left\{\alpha_{n}\right\},\left\{\beta_{n}\right\}$, $\left\{\gamma_{n}\right\},\left\{\delta_{n}\right\}$, and $\left\{\sigma_{n}\right\}$ are the sequences in $(0,1)$ such that $\alpha_{n}+\beta_{n}+$ $\gamma_{n}+\delta_{n}=1$ for all $n \geq 0$. Suppose that the following conditions hold:

(i) $\lim _{n \rightarrow \infty} \alpha_{n}=0$ and $\sum_{n=0}^{\infty} \alpha_{n}=\infty$;

(ii) $\left\{\gamma_{n}\right\},\left\{\delta_{n}\right\} \subset[c, d]$ for some $c, d \in(0,1)$;

(iii) $\sum_{n=1}^{\infty}\left(\left|\sigma_{n}-\sigma_{n-1}\right|+\left|\alpha_{n}-\alpha_{n-1}\right|+\left|\beta_{n}-\beta_{n-1}\right|+\left|\gamma_{n}-\gamma_{n-1}\right|+\right.$ $\left.\left|\delta_{n}-\delta_{n-1}\right|\right)<\infty$;

(iv) $0<\liminf _{n \rightarrow \infty} \beta_{n} \leq \lim \sup _{n \rightarrow \infty} \beta_{n}<1$ and $0<$ $\liminf _{n \rightarrow \infty} \sigma_{n} \leq \limsup _{n \rightarrow \infty} \sigma_{n}<1$.

Assume that $\sum_{n=1}^{\infty} \sup _{x \in D}\left\|S_{n} x-S_{n-1} x\right\|<\infty$ for any bounded subset $D$ of $C$ and let $S$ be a mapping of $C$ into itself defined by $S x=\lim _{n \rightarrow \infty} S_{n} x$ for all $x \in C$ and suppose that $\operatorname{Fix}(S)=$ $\bigcap_{i=0}^{\infty} \operatorname{Fix}\left(S_{i}\right)$. Then, there hold the following:

(I) $\lim _{n \rightarrow \infty}\left\|x_{n+1}-x_{n}\right\|=0$;

(II) the sequence $\left\{x_{n}\right\}_{n=0}^{\infty}$ converges strongly to some $q \epsilon$ $F$ which is the unique solution of the variational inequality problem (VIP)

$$
\langle(I-f) q, J(q-p)\rangle \leq 0, \quad \forall p \in F,
$$

provided $\beta_{n} \equiv \beta$ for some fixed $\beta \in(0,1)$.

Proof. In Theorem 33, we put $B_{1}=I-V, B_{2}=0$ and $\mu_{1}=l$ where $1-(\zeta /(1+\zeta))(1-\sqrt{(1-\theta) / \zeta}) \leq l \leq 1$. Then, GSVI (13) is equivalent to the VIP of finding $x^{*} \in C$ such that

$$
\left\langle B_{1} x^{*}, J\left(x-x^{*}\right)\right\rangle \geq 0, \quad \forall x \in C .
$$

In this case, $B_{1}: C \rightarrow X$ is $\zeta$-strictly pseudocontractive and $\theta$-strongly accretive. Repeating the same arguments as those in the proof of Corollary 25, we can infer that $\operatorname{Fix}(V)=\operatorname{VI}\left(C, B_{1}\right)$. Accordingly, $F=\left(\bigcap_{i=0}^{\infty} \operatorname{Fix}\left(S_{i}\right)\right) \cap \Omega \cap$ $\left(\bigcap_{i=0}^{\infty} \operatorname{VI}\left(C, A_{i}\right)\right)=\left(\bigcap_{i=0}^{\infty} \operatorname{Fix}\left(S_{i}\right)\right) \cap \operatorname{Fix}(V) \cap\left(\bigcap_{i=0}^{\infty} \operatorname{VI}\left(C, A_{i}\right)\right)$,

$$
G x_{n}=((1-l) I+l V) x_{n}
$$

So, the scheme (251) reduces to (306). Therefore, the desired result follows from Theorem 33 .

Remark 35. Our Theorems 31 and 33 improve, extend, supplement and develop Ceng and Yao's [10, Theorem 3.2], Cai and Bu's [11, Theorem 3.1], Kangtunyakarn's [38, Theorem 3.1], and Ceng and Yao's [8, Theorem 3.1], in the following aspects. 
(i) The problem of finding a point $q \in\left(\bigcap_{i=0}^{\infty} \operatorname{Fix}\left(S_{i}\right)\right) \cap$ $\Omega \cap\left(\bigcap_{i=0}^{\infty} \operatorname{VI}\left(C, A_{i}\right)\right)$ in our Theorems 31 and 33 is more general and more subtle than every one of the problem of finding a point $q \in \bigcap_{i=0}^{\infty} \operatorname{Fix}\left(T_{i}\right)$ in $[10$, Theorem 3.2], the problem of finding a point $q \in$ $\bigcap_{i=1}^{\infty} \operatorname{Fix}\left(T_{i}\right) \cap \Omega$ in [11, Theorem 3.1], the problem of finding a point $q \in \operatorname{Fix}(S) \cap \operatorname{Fix}(V) \cap\left(\bigcap_{i=1}^{N} \operatorname{VI}\left(C, A_{i}\right)\right)$ in [38, Theorem 3.1], and the problem of finding a point $q \in \operatorname{Fix}(T)$ in [8, Theorem 3.1].

(ii) The iterative scheme in [38, Theorem 3.1] is extended to develop the iterative scheme (178) of our Theorem 31, and the iterative scheme in [11, Theorem 3.1] is extended to develop the iterative scheme (251) of our Theorem 33. Iterative schemes (178) and (181) in our Theorems 31 and 33 are more advantageous and more flexible than the iterative scheme of [11, Theorem 3.1] because they both are one-step iteration schemes and involve several parameter sequences $\left\{\alpha_{n}\right\},\left\{\beta_{n}\right\},\left\{\gamma_{n}\right\}$, $\left\{\delta_{n}\right\}$, (and $\left.\left\{\sigma_{n}\right\}\right)$.

(iii) Our Theorems 31 and 33 extend and generalize Ceng and Yao's [8, Theorem 3.1] from a nonexpansive mapping to a countable family of nonexpansive mappings, and Ceng and Yao's [10, Theorems 3.2] to the setting of the GSVI (13) and infinitely many VIPs, Kangtunyakarn's [38, Theorem 3.1] from finitely many VIPs to infinitely many VIPs, from a nonexpansive mapping to a countable family of nonexpansive mappings and from a strict pseudocontraction to the GSVI (13). In the meantime, our Theorems 31 and 33 extend and generalize Cai and Bu's [11, Theorem 3.1] to the setting of infinitely many VIPs.

(iv) The iterative schemes (178) and (251) in our Theorems 31 and 33 are very different from every one in [10, Theorem 3.2], [11, Theorem 3.1], [38, Theorem 3.1], and [8, Theorem 3.1] because the mappings $G$ and $T_{n}$ in [11, Theorem 3.1] and the mapping $T$ in $[8$, Theorem 3.1] are replaced with the same composite mapping $S_{n} G$ in the iterative schemes (42) and (130) and the mapping $W_{n}$ in [10, Theorem 3.2] is replaced by $B_{n}$.

(v) Cai and Bu's proof in [11, Theorem 3.1] depends on the argument techniques in [14], the inequality in 2uniformly smooth Banach spaces (see Lemma 4), and the inequality in smooth and uniform convex Banach spaces (see Proposition 6). Because the composite mapping $S_{n} G$ appears in the iterative scheme (178) of our Theorem 31, the proof of our Theorem 31 depends on the argument techniques in [14], the inequality in 2-uniformly smooth Banach spaces (see Lemma 4), the inequality in smooth and uniform convex Banach spaces (see Proposition 6), the inequalities in uniform convex Banach spaces (see Lemmas 11 and 15 in Section 2 of this paper), and the properties of the $W$-mapping and the Banach limit (see Lemmas 16, 17, and 18 in Section 2 of this paper). However, the proof of our Theorem 33 does not depend on the argument techniques in [14], the inequality in 2uniformly smooth Banach spaces (see Lemma 4), and the inequality in smooth and uniform convex Banach spaces (see Proposition 6). It depends on only the inequalities in uniform convex Banach spaces (see Lemmas 11 and 15 in Section 2 of this paper) and the properties of the $W$-mapping and the Banach limit (see Lemmas 16-18 in Section 2 of this paper).

(vi) The assumption of the uniformly convex and 2uniformly smooth Banach space $X$ in [11, Theorem 3.1] is weakened to the one of the uniformly convex Banach space $X$ having a uniformly Gateaux differentiable norm in our Theorem 33. Moreover, the assumption of the uniformly smooth Banach space $X$ in [8, Theorem 3.1] is replaced with the one of the uniformly convex Banach space $X$ having a uniformly Gateaux differentiable norm in our Theorem 33.

\section{Conflict of Interests}

The authors declare that there is no conflict of interests regarding the publication of this paper.

\section{Acknowledgments}

This work was funded by the Deanship of Scientific Research (DSR), King Abdulaziz University under Grant no. HiCi/15130-1433. The authors, therefore, acknowledge technical and financial support of KAU. The authors would like to thank Professor J. C. Yao for motivation and many fruitful discussions regarding this work.

\section{References}

[1] F. E. Browder, "Convergence theorems for sequences of nonlinear operators in Banach spaces," Mathematische Zeitschrift, vol. 100, pp. 201-225, 1967.

[2] L. C. Zeng, G. M. Lee, and N. C. Wong, "Ishikawa iteration with errors for approximating fixed points of strictly pseudocontractive mappings of Browder-Petryshyn type," Taiwanese Journal of Mathematics, vol. 10, no. 1, pp. 87-99, 2006.

[3] H. Iiduka and W. Takahashi, "Strong convergence theorems for nonexpansive mappings and inverse-strongly monotone mappings," Nonlinear Analysis, vol. 61, no. 3, pp. 341-350, 2005.

[4] W. Takahashi and M. Toyoda, "Weak convergence theorems for nonexpansive mappings and monotone mappings," Journal of Optimization Theory and Applications, vol. 118, no. 2, pp. 417428, 2003.

[5] H. Iiduka, W. Takahashi, and M. Toyoda, "Approximation of solutions of variational inequalities for monotone mappings," Panamerican Mathematical Journal, vol. 14, no. 2, pp. 49-61, 2004.

[6] Y. Takahashi, K. Hashimoto, and M. Kato, "On sharp uniform convexity, smoothness, and strong type, cotype inequalities," Journal of Nonlinear and Convex Analysis, vol. 3, no. 2, pp. 267281, 2002.

[7] Y. Yao, R. Chen, and J. C. Yao, "Strong convergence and certain control conditions for modified Mann iteration," Nonlinear Analysis, vol. 68, no. 6, pp. 1687-1693, 2008. 
[8] L. C. Ceng and J. C. Yao, "Convergence and certain control conditions for hybrid viscosity approximation methods," Nonlinear Analysis, vol. 73, no. 7, pp. 2078-2087, 2010.

[9] J. G. O’Hara, P. Pillay, and H. K. Xu, "Iterative approaches to convex feasibility problems in Banach spaces," Nonlinear Analysis, vol. 64, no. 9, pp. 2022-2042, 2006.

[10] L. C. Ceng and J. C. Yao, "Relaxed viscosity approximation methods for fixed point problems and variational inequality problems," Nonlinear Analysis, vol. 69, no. 10, pp. 3299-3309, 2008.

[11] G. Cai and S. Bu, "Convergence analysis for variational inequality problems and fixed point problems in 2-uniformly smooth and uniformly convex Banach spaces," Mathematical and Computer Modelling, vol. 55, no. 3-4, pp. 538-546, 2012.

[12] R. U. Verma, "On a new system of nonlinear variational inequalities and associated iterative algorithms," Mathematical Sciences Research Hot-Line, vol. 3, no. 8, pp. 65-68, 1999.

[13] J. L. Lions and G. Stampacchia, "Variational inequalities," Communications on Pure and Applied Mathematics, vol. 20, pp. 493-519, 1967.

[14] L. C. Ceng, C. Y. Wang, and J. C. Yao, "Strong convergence theorems by a relaxed extragradient method for a general system of variational inequalities," Mathematical Methods of Operations Research, vol. 67, no. 3, pp. 375-390, 2008.

[15] G. M. Korpelevič, "An extragradient method for finding saddle points and for other problems," Ėkonomika i Matematicheskie Metody, vol. 12, no. 4, pp. 747-756, 1976.

[16] F. Facchinei and J. S. Pang, Finite-Dimensional Variational Inequalities and Complementarity Problems, vol. 1-2, Springer, New York, NY, USA, 2003.

[17] A. N. Iusem and B. F. Svaiter, "A variant of Korpelevich's method for variational inequalities with a new search strategy," Optimization, vol. 42, no. 4, pp. 309-321, 1997.

[18] L. C. Ceng and J. C. Yao, "An extragradient-like approximation method for variational inequality problems and fixed point problems," Applied Mathematics and Computation, vol. 190, no. 1, pp. 205-215, 2007.

[19] M. V. Solodov and B. F. Svaiter, "A new projection method for variational inequality problems," SIAM Journal on Control and Optimization, vol. 37, no. 3, pp. 765-776, 1999.

[20] Y. Censor, A. Gibali, and S. Reich, "Two extensions of Korpelevich's extragradient method for solving the variational inequality problem in Euclidean space," Tech. Rep., 2010.

[21] L. C. Zeng and J. C. Yao, "Strong convergence theorem by an extragradient method for fixed point problems and variational inequality problems," Taiwanese Journal of Mathematics, vol. 10, no. 5, pp. 1293-1303, 2006.

[22] N. Nadezhkina and W. Takahashi, "Weak convergence theorem by an extragradient method for nonexpansive mappings and monotone mappings," Journal of Optimization Theory and Applications, vol. 128, no. 1, pp. 191-201, 2006.

[23] L. C. Ceng, Q. H. Ansari, and J. C. Yao, "An extragradient method for solving split feasibility and fixed point problems," Computers \& Mathematics with Applications, vol. 64, no. 4, pp. 633-642, 2012.

[24] L. C. Ceng, M. Teboulle, and J. C. Yao, "Weak convergence of an iterative method for pseudomonotone variational inequalities and fixed-point problems," Journal of Optimization Theory and Applications, vol. 146, no. 1, pp. 19-31, 2010.

[25] L. C. Ceng, Q. H. Ansari, and J. C. Yao, "Relaxed extragradient methods for finding minimum-norm solutions of the split feasibility problem," Nonlinear Analysis, vol. 75, no. 4, pp. $2116-$ 2125, 2012.

[26] L. C. Ceng, Q. H. Ansari, and J. C. Yao, "Relaxed extragradient iterative methods for variational inequalities," Applied Mathematics and Computation, vol. 218, no. 3, pp. 1112-1123, 2011.

[27] K. Aoyama, Y. Kimura, W. Takahashi, and M. Toyoda, "Approximation of common fixed points of a countable family of nonexpansive mappings in a Banach space," Nonlinear Analysis, vol. 67, no. 8, pp. 2350-2360, 2007.

[28] R. U. Verma, "Projection methods, algorithms, and a new system of nonlinear variational inequalities," Computers \& Mathematics with Applications, vol. 41, no. 7-8, pp. 1025-1031, 2001.

[29] L. C. Ceng, Q. H. Ansari, N. C. Wong, and J. C. Yao, "An extragradient-like approximation method for variational inequalities and fixed point problems," Fixed Point Theory and Applications, vol. 2011, article 22, 18 pages, 2011.

[30] L. C. Ceng, N. Hadjisavvas, and N. C. Wong, "Strong convergence theorem by a hybrid extragradient-like approximation method for variational inequalities and fixed point problems," Journal of Global Optimization, vol. 46, no. 4, pp. 635-646, 2010.

[31] L. C. Ceng, S. M. Guu, and J. C. Yao, "Finding common solutions of a variational inequality, a general system of variational inequalities, and a fixed-point problem via a hybrid extragradient method," Fixed Point Theory and Applications, vol. 2011, Article ID 626159, 22 pages, 2011.

[32] L. C. Ceng and A. Petruşel, "Krasnoselski-Mann iterations for hierarchical fixed point problems for a finite family of nonself mappings in Banach spaces," Journal of Optimization Theory and Applications, vol. 146, no. 3, pp. 617-639, 2010.

[33] Y. Yao, Y. C. Liou, S. M. Kang, and Y. Yu, "Algorithms with strong convergence for a system of nonlinear variational inequalities in Banach spaces," Nonlinear Analysis, vol. 74, no. 17, pp. 60246034, 2011.

[34] K. Aoyama, H. Iiduka, and W. Takahashi, "Weak convergence of an iterative sequence for accretive operators in Banach spaces," Fixed Point Theory and Applications, vol. 2006, Article ID 35390, 13 pages, 2006.

[35] J. S. Jung, "Iterative approaches to common fixed points of nonexpansive mappings in Banach spaces," Journal of Mathematical Analysis and Applications, vol. 302, no. 2, pp. 509-520, 2005.

[36] L. C. Ceng, H. K. Xu, and J. C. Yao, "Strong convergence of an iterative method with perturbed mappings for nonexpansive and accretive operators," Numerical Functional Analysis and Optimization, vol. 29, no. 3-4, pp. 324-345, 2008.

[37] S. Kamimura and W. Takahashi, "Weak and strong convergence of solutions to accretive operator inclusions and applications," Set-Valued Analysis Devoted to the Theory of Multifunctions and Its Applications, vol. 8, no. 4, pp. 361-374, 2000.

[38] A. Kangtunyakarn, "Iterative scheme for a nonexpansive mapping, an $\eta$-strictly pseudo-contractive mapping and variational inequality problems in a uniformly convex and 2-uniformly smooth Banach space," Fixed Point Theory and Applications, vol. 2013, article 23, 21 pages, 2013.

[39] H. K. Xu, "Inequalities in Banach spaces with applications," Nonlinear Analysis, vol. 16, no. 12, pp. 1127-1138, 1991.

[40] S. Kamimura and W. Takahashi, "Strong convergence of a proximal-type algorithm in a Banach space," SIAM Journal on Optimization, vol. 13, no. 3, pp. 938-945, 2002.

[41] H. K. Xu, "Iterative algorithms for nonlinear operators," Journal of the London Mathematical Society, vol. 66, no. 1, pp. 240-256, 2002 . 
[42] S. S. Chang, "Some problems and results in the study of nonlinear analysis," Nonlinear Analysis, vol. 30, no. 7, pp. 41974208.

[43] S. Reich, "Weak convergence theorems for nonexpansive mappings in Banach spaces," Journal of Mathematical Analysis and Applications, vol. 67, no. 2, pp. 274-276, 1979.

[44] Y. J. Cho, H. Zhou, and G. Guo, "Weak and strong convergence theorems for three-step iterations with errors for asymptotically nonexpansive mappings," Computers \& Mathematics with Applications, vol. 47, no. 4-5, pp. 707-717, 2004.

[45] K. Aoyama, Y. Kimura, W. Takahashi, and M. Toyoda, "Approximation of common fixed points of a countable family of nonexpansive mappings in a Banach space," Nonlinear Analysis, vol. 67 , no. 8, pp. 2350-2360, 2007.

[46] H. K. Xu, "Viscosity approximation methods for nonexpansive mappings," Journal of Mathematical Analysis and Applications, vol. 298, no. 1, pp. 279-291, 2004.

[47] R. E. Bruck, Jr., "Properties of fixed-point sets of nonexpansive mappings in Banach spaces," Transactions of the American Mathematical Society, vol. 179, pp. 251-262, 1973.

[48] K. Shimoji and W. Takahashi, "Strong convergence to common fixed points of infinite nonexpansive mappings and applications," Taiwanese Journal of Mathematics, vol. 5, no. 2, pp. 387404, 2001.

[49] H. Zhou, L. Wei, and Y. J. Cho, "Strong convergence theorems on an iterative method for a family of finite nonexpansive mappings in reflexive Banach spaces," Applied Mathematics and Computation, vol. 173, no. 1, pp. 196-212, 2006.

[50] N. Shioji and W. Takahashi, "Strong convergence of approximated sequences for nonexpansive mappings in Banach spaces," Proceedings of the American Mathematical Society, vol. 125, no. 12, pp. 3641-3645, 1997.

[51] T. Suzuki, "Strong convergence of Krasnoselskii and Mann's type sequences for one-parameter nonexpansive semigroups without Bochner integrals," Journal of Mathematical Analysis and Applications, vol. 305, no. 1, pp. 227-239, 2005. 


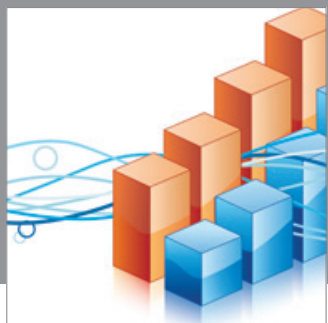

Advances in

Operations Research

mansans

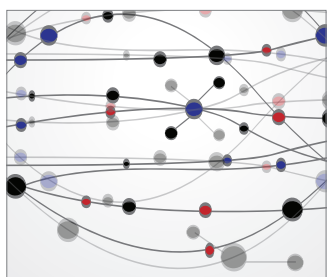

The Scientific World Journal
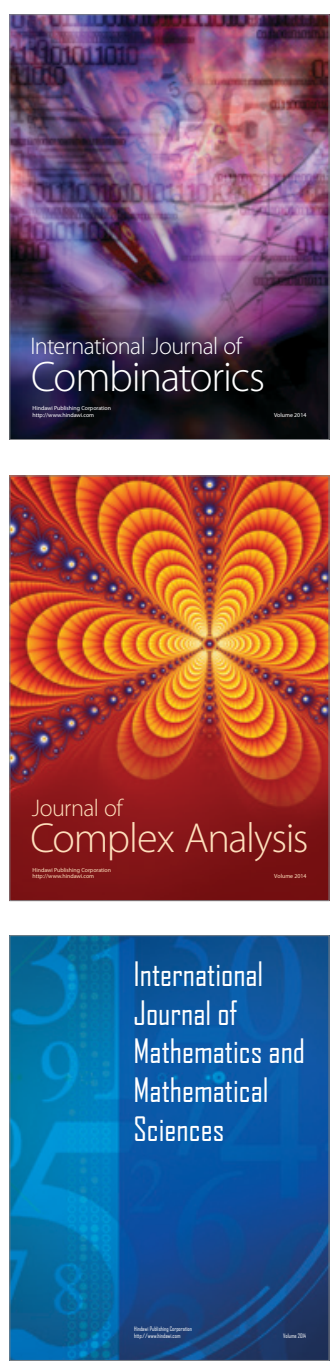
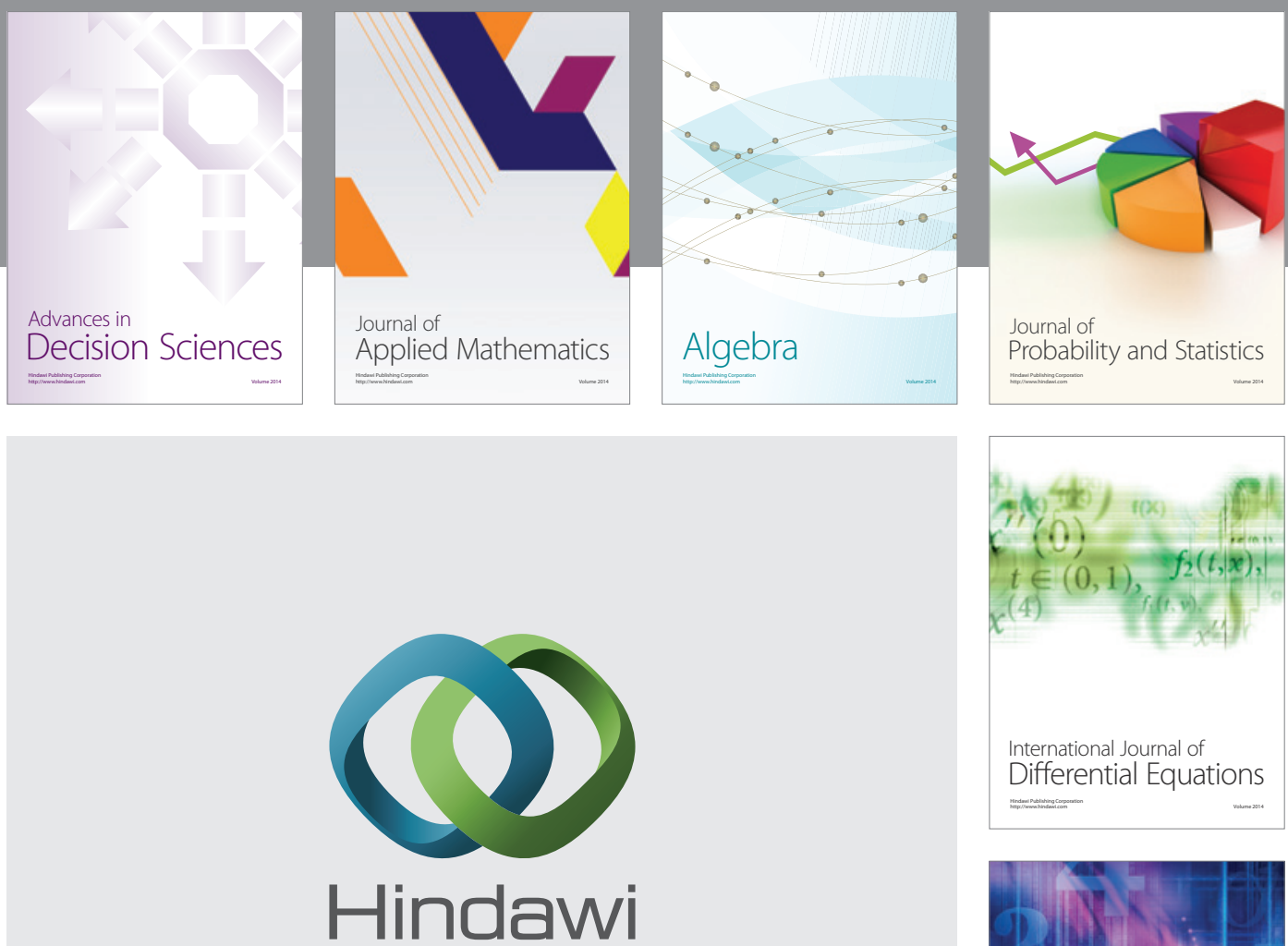

Submit your manuscripts at http://www.hindawi.com
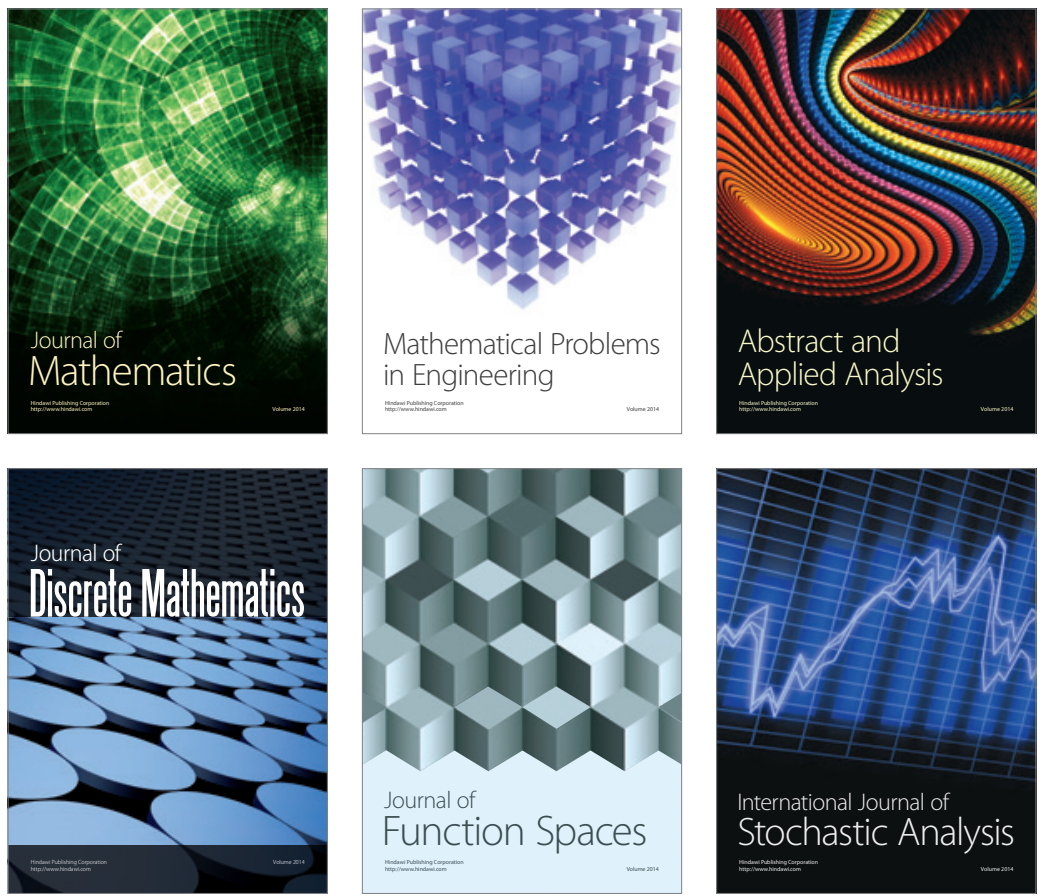

Journal of

Function Spaces

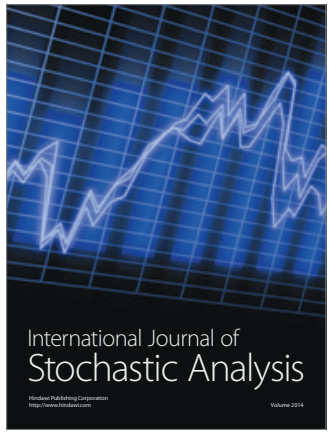

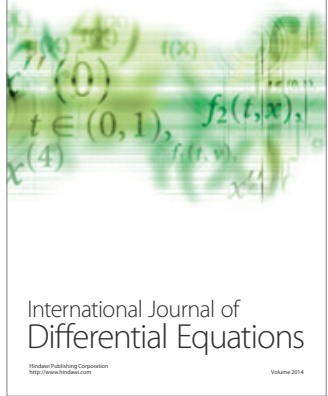
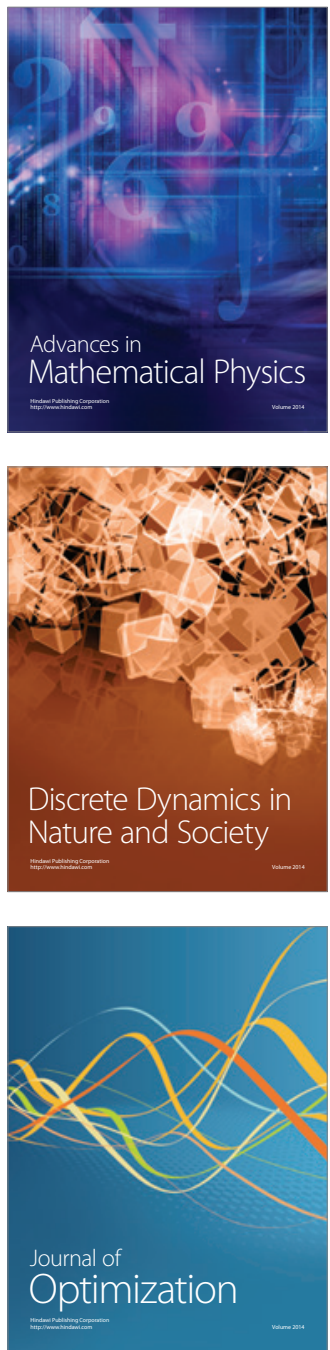